DEFECT DETECTION IN SELECTIVE LASER MELTING

A Thesis

presented to

the Faculty of California Polytechnic State University,

San Luis Obispo

In Partial Fulfillment

of the Requirements for the Degree

Master of Science in Mechanical Engineering

by

Moira Foster

June 2018 
(C) 2018

Moira Foster

ALL RIGHTS RESERVED 
TITLE: Defect Detection in Selective Laser Melting

AUTHOR: $\quad$ Moira Foster

DATE SUBMITTED: June 2018

COMMITTEE CHAIR: $\quad$ Peter Schuster, Ph.D.

Professor of Mechanical Engineering

COMMITTEE MEMBER: Xuan Wang, Ph.D.

Professor of Manufacturing Engineering

COMMITTEE MEMBER: Charles Birdsong, Ph.D.

Professor of Mechanical Engineering 


\title{
ABSTRACT \\ Defect Detection in Selective Laser Melting
}

\author{
Moira Foster
}

Additively manufactured parts produced using selective laser melting (SLM) are prone to defects created during the build process due to part shrinkage while cooling. Currently defects are found only after the part is removed from the printer. To determine whether cracks can be detected before a print is completed, this project developed print parameters to print a test coupon with inherent defects - warpage and cracking. Data recorded during the build was then characterized to determine when the defects occurred.

The test coupon was printed using two sets of print parameters developed to control the severity of warpage and cracking. The builds were monitored using an accelerometer recording at 12500 samples per second, an iphone recording audio at 48000 samples a second, and a camera taking a photo every build layer. Data was analyzed using image comparison, signal amplitude, Fourier Transform, and Wavelet Decomposition.

The developed print parameters reduced warpage in the part by better distributing heat throughout the build envelope. Reducing warpage enabled the lower portion of the part to be printed intact, preserving it to experience cracking later in the build. From physical evidence on the part as well as time stamps from the machine script, several high energy impulse events in the accelerometer data were determined to be when cracking occurred in the build. This project's preliminary investigation of accelerometers to detect defects in selective laser melting will be used in future work to create machine learning algorithms that would control the machine in real time and address defects as they arise.

Keywords: Additive Manufacturing, Selective Laser Melting, Accelerometer, Cracking, Warping, Print Parameters, Defect, Detection 


\section{ACKNOWLEDGMENTS}

Thank you to everyone on my committee - Dr. Xuan Wang, Dr. Peter Schuster, and Dr. Charles Birdsong - for their tireless support of this project. It was helpful to have a diverse and supportive board to turn to throughout the process. Thank you to LLNL's team - Matt Wraith, Stephen Knaus, and Tommy Pluschkell - for their sponsorship and advice through the printing process. LLNL funded the material and machine costs of the project. Also thank you to Hajime Yamanaka for being a great co-operator on the SLM and for all his material knowledge he supplied to the project. Jeff Zimmerman graciously supported me by bouncing off ideas regarding accelerometer analysis and data collection. Lastly thank you to Gaby Dinata, Nicholas Crawford, and the rest of my family for their support through the editing process. 


\section{TABLE OF CONTENTS}

$\begin{array}{lll}\text { Page } & \text { Pag } \\ & & \end{array}$

List of Tables .............................................................................................. viii

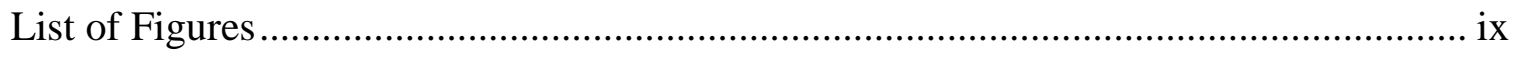

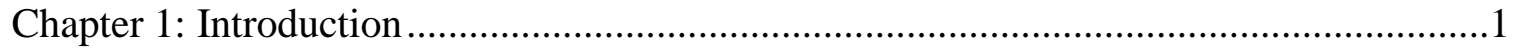

1.1 Selective Laser Melting Process .......................................................................... 2

1.2 SLM Solutions 125 Hardware Overview ........................................................... 3

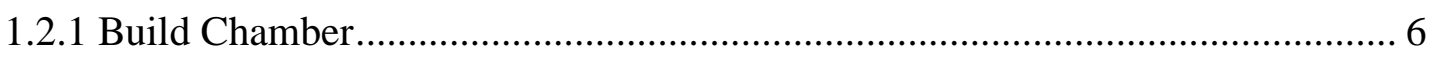

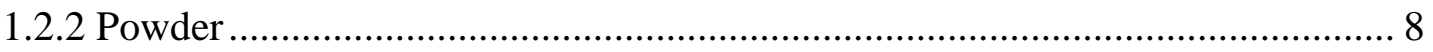

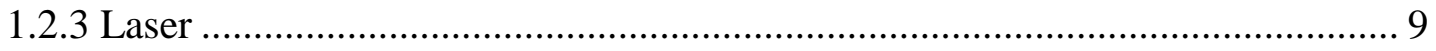

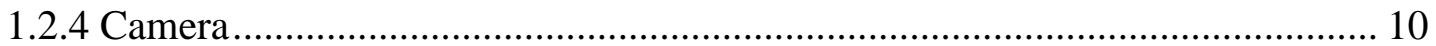

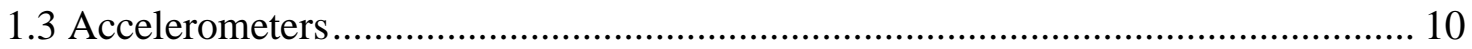

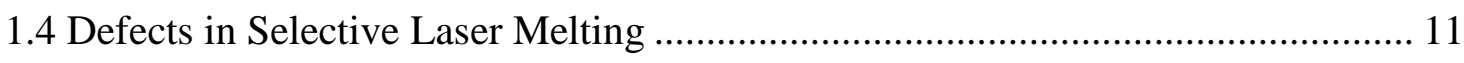

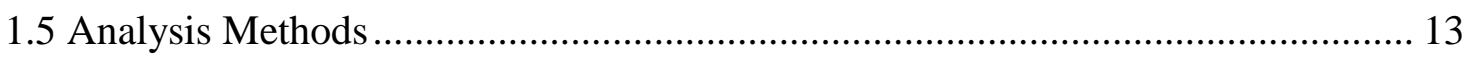

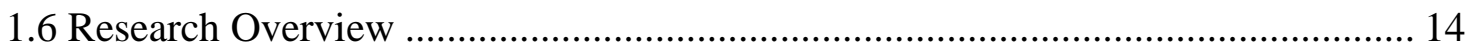

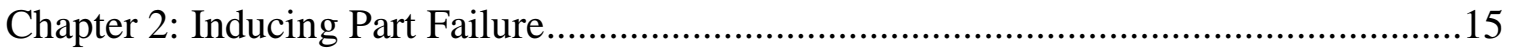

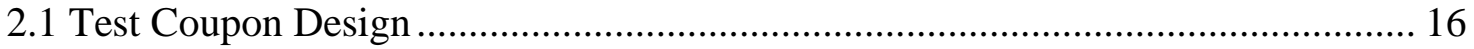

2.2 Print Parameters Development...................................................................... 17

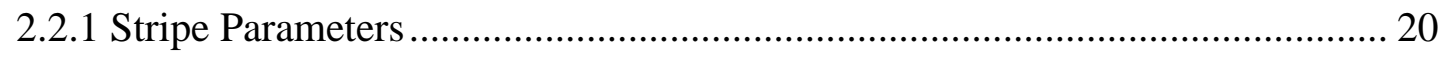

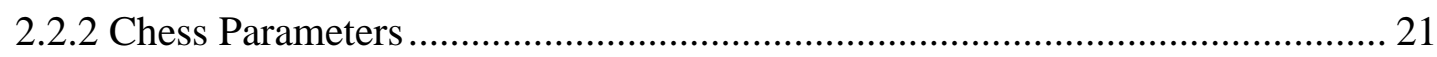

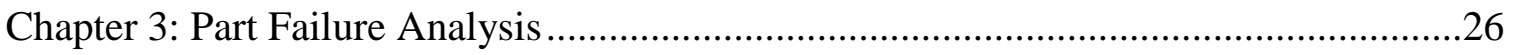

3.1 Warpage in Narrowly Supported Cross Sections ............................................. 26

3.2 Cracking in Narrow Section Due to Heat........................................................ 30

3.3 Material Failure Modes ................................................................................... 32

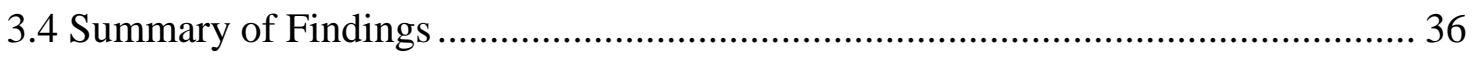

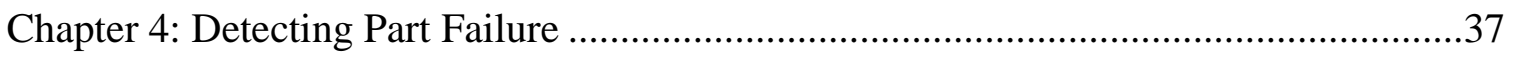

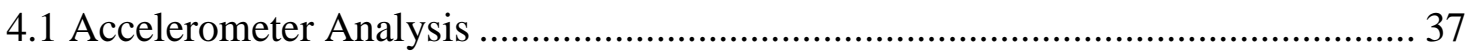

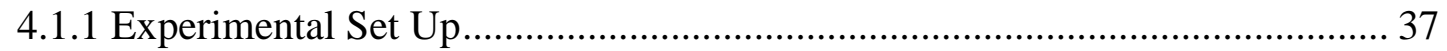

4.1.2 Frequency Analysis Methods Overview..................................................... 41

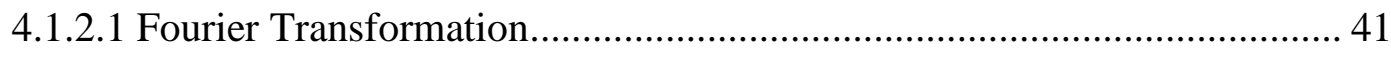

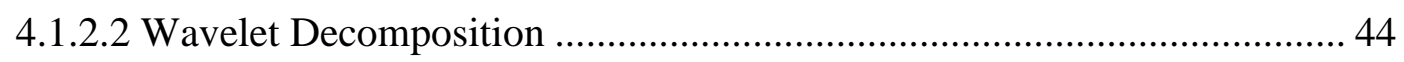


4.1.2.3 Shock Response Spectrum...................................................................... 47

4.1.3 Print Profile Overview ............................................................................ 50

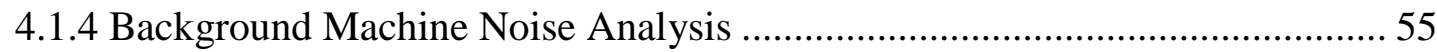

4.1.5 Warpage Defect Signature ........................................................................ 58

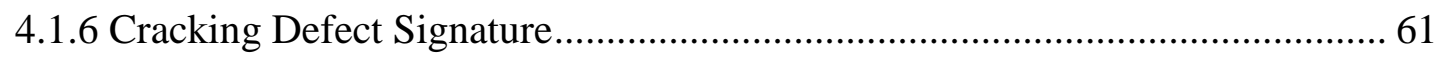

4.1.6.1 Evidence to Support Cracking Event........................................................... 62

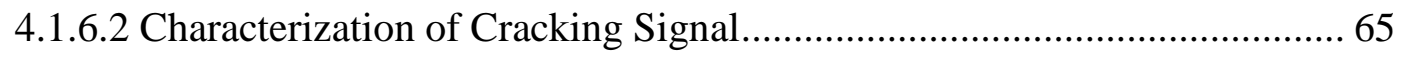

4.1.7 FEA Modal Frequency Simulation .................................................................. 72

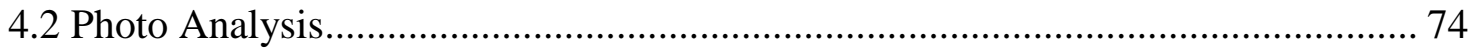

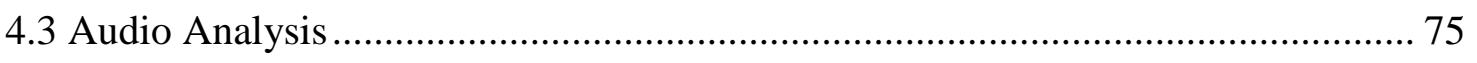

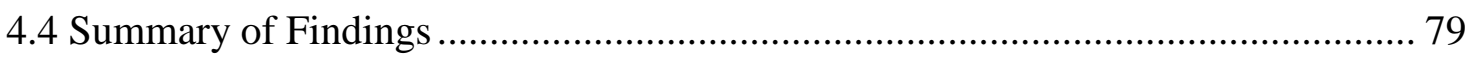

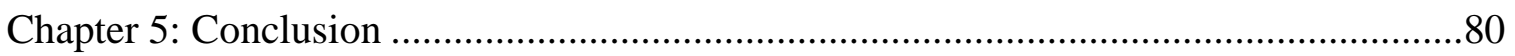

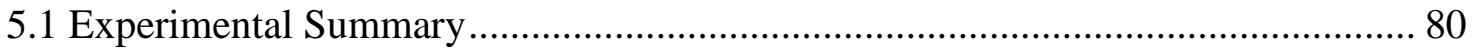

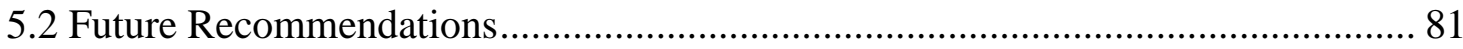

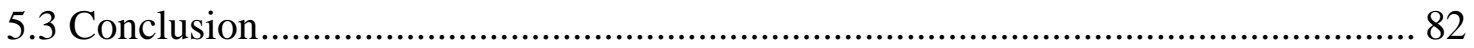

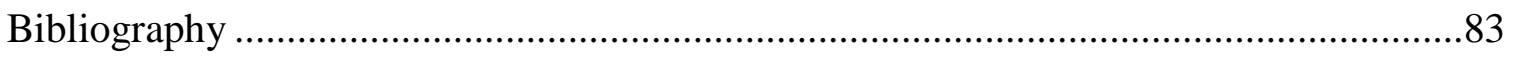

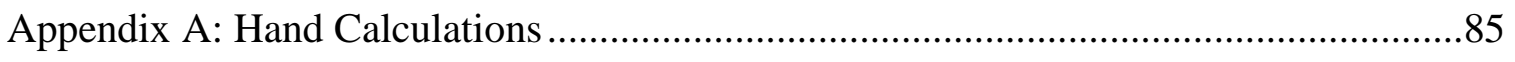

Appendix B: Test Coupon Drawings.............................................................................

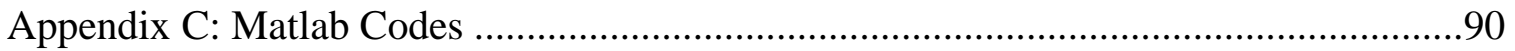




\section{LIST OF TABLES}

Table

1. There are various print parameters that determine the quality of the build. Some of the parameters are listed below with their units. These parameters are further defined in Chapter 2 ................................................................................ 9

2. A summary of print parameter definitions, some are visually defined below...... 18

3. Key Stripe Print Parameters................................................................................... 21

4. Differences between Concept Laser print parameters at LLNL and SLM Solutions' default stripe parameters used in the first builds at Cal Poly.

5. Important chess print parameters used when printing the test coupons are summarized alongside the default stripe parameter settings.

6. The frequencies excited by the three potential cracks are summarized in the table. The frequencies were selected as the center of the hot yellow spots on the wavelet plots.

7. The frequencies excited by various events are summarized below for comparison. The cracks $(\mathrm{C})$ have higher frequencies in general than the hammer $(\mathrm{H})$ and recoater $(\mathrm{R})$. 


\section{LIST OF FIGURES}

Figure

1. A diagram of the SLM printing process. Metal powder is spread in a thin layer across a metal plate (a). A laser the traces out the cross section of the part (b). The plate is then lowered to the thickness of a layer of powder and the process repeats (c) (Cai, Malcolm and Wong).............................................................. 3

2. Photograph of the SLM Solutions $125 \mathrm{HL}$. The printer has a build volume of $125 \times 125 \times 125 \mathrm{~mm}^{3}$. The machine includes powder inlet (1), build chamber (2), and the computer (3).

3. Photograph of the SLM Sieving Station that removes large irregular particles from the powder so the powder can be reused in the next build

4. A SEM photo reveals irregular conglomerates of particles (A) are created when they are heated enough to partially fuse together. Heat affected particles (B) occur when the particle is melted multiple times. Normal Particles (C) are separate and free flowing.

5. As seen in the photo taken through the build chamber window, the build chamber contains (1) the powder hopper, (2) the recoater, (3) the build plate....... 6

6. CAD model of a SLM Solutions recoater. The recoater has a twin rubber blade to allow for even spreading of powder in both directions across the build. The recoater can hold two layers of powder, allowing it only to refill at the hopper at the back of the machine. (SLM Solutions Group)

7. The labeled photo of the inside of the build chamber shows that the build plate lowers down as each layer is added to the part (1). The recoater blade moves from front to back of the machine to add new layer of powder (2). The Argon flows from right to left to maintain the atmosphere and move ash away from the part (3).

8. The diagram of the accelerometer connections shows the accelerometers are attached to the bottom of the build platform using silicone adhesive. The cables exit the SLM Solutions machine through a hole in the top of the machine for the inlet of the powder. The cables are then connected to the DAQ.

9. These photos demonstrate warpage can negatively impact later layers of the part through destruction to the recoater blade. The pieces of rubber missing from the recoater blade (a) lead to unevenness in powder spread. Thicker layers in the powder spread translate to thicker regions in the build layers. These thicker regions produce nonuniform part geometry (b) and material properties.

10. The two defects produced in the test coupons were (left) warpage and (right) cracking as seen in the photos. Warpage features the remains of the lower leg with the upper leg pulled off. The upper leg was pulled off by the recoater hitting into its warpage. The burning on the top are of the leg is due to the laser melting loose powder. The loose powder is pushed by the recoater as it 
is not attached rigidly. This results in the burning appearance of the test coupon.

11. A photograph of the experimental part contains two legs with weak cracking locations (a), a middle support leg (b), and an upper high heat portion (c).......... 16

12. Common Materialise Magics Print Parameter Terminology. (Materialise) ......... 19

13. A stripe hatch pattern means the laser crosses the entire width of the part before beginning the next stripe. (Rensihaw)

14. A diagram of the chess pattern consists of squares of stripe hatches. These squares are traced out by the laser in a random pattern to better distribute the heat. (Rensihaw).

15. The chess hatch pattern is seen in a microscope image of the part surface

16. Photograph of the surface of the powder layer during warpage showing the corners of the leg warped upward (a). The warpage had previously ripped the recoater blade, resulting in uneven powder spread in subsequent layers as seen by the lines in the powder (b).

17. Photograph of a recoater blade damaged from hitting against sharp upturned corners of warpage.

18. A photograph of a part printed past warpage demonstrates how a torn recoater blade can result in uneven powder spread. The layer boundaries in the part are no longer horizontal or even. The layers in the circle are lower than the surrounding layers.

19. During the build, a piece is seen to be disconnected from the test coupon in a photo of the top powder layer. In this picture, the leg is pushed on the backwards recoat completely out of its original position.

20. A photograph of the top powder layer during the build reveals the void, left from the moved test coupon piece, remains hot longer than the other test coupons of the build.

21. A photograph of the completed test coupon. The upper portion of the test coupon shrinks during cooling (a), inducing a crack in the lower weak section of the test coupon (b). The crack relieves thermally induced stress in both the upper portion and lower portion of the test coupon due to inconsistency during shrinkage.

22. In the photograph of the completed test coupon, a shift is visible at the layer that the test coupon cracked. The line is due to the test coupon moving during the build due to the crack event without the printer's knowledge.

23. A Micro-vu image of a leg shows the difference in thickness between the upper and lower layers. The upper layers have undergone plastic deformation and are elongated, reducing their thickness. 
24. A photograph of the remaining leg stub from the severe warpage cases suggests that it is bent slowly over time, allowing it to undergo plastic deformation.

25. The SEM photos of the crack surface shows that the failure was a brittle (a) to ductile (b) tensile failure. (Yamanaka)

26. A SEM image shows the irregular conglomerates of particles (A) are created when they are heated enough to partially fuse together. Heat affected particles (B) occur when the particle is melted multiple times. Normal Particles (C) are separate and free flowing............Error! Bookmark not defined.

27. The accelerometer's location on the printer is compared with its location in the CAD model. The accelerometers were mounted using silicone adhesive. The orientation of the triaxial accelerometer has the $\mathrm{x}$ axis in the upwards direction. The $\mathrm{y}$ axis is skewed 45 degrees clockwise about the $\mathrm{x}$ axis. (SLM Solutions Group)

28. Three acceleration versus time plots recording the same event in time are compared. The triaxial accelerometer measured a tap of the modal hammer in all three directions with similar magnitude.

29. Two example signals, green and blue, are compared in the time domain. Aliasing is a problem that can occur in signal analysis when a sample rate is too low to obtain the actual shape of the input data. In the above example, the green samples do not adequately show the true shape of the blue input data. (Wickert)

30. An acceleration versus time plot of a noise test demonstrated that movements produced by unrelated noises would not produce a signal above the amplitude of the normal operating machine noise which is $0.05 \mathrm{~g}$

31. The Fourier Transform of a signal results in a magnitude vs. frequency plot...... 43

32. A phase spectrum displays the time delay of each frequency. The unique combination of phase and magnitude of different frequencies creates an individual signal.

33. A time domain plot of the signal with a Morse Wavelet superimposed (left) is then transformed into a frequency versus time domain plot (right) using Wavelet Decomposition. The Morse Wavelet can match a signal response by varying the gamma and $\mathrm{P}$ parameters. (MathWorks)

34. The time domain plot of a spike is transformed into the frequency vs. time plot by the function cwt.m. The frequency axis of the Wavelet Plot is a log scale.....

35. The SRS model uses multiple mass-spring dampers to simulate the excitation of different natural frequencies (Irvine)...

36. Plot of acceleration versus time with low amplitude filter applied for data collected during a build with severer warpage. 
37. Plot of acceleration versus time with low amplitude filter applied for data collected during the cracked build. The build was paused just before 500 minutes and then resumed later. The remaining resumed build data is not shown. The accelerometer profile for the first part of the build has five parts based off the amplitude of the raw accelerometer data: beginning recoater, warpage, later recoater, crack, and machine paused.

38. A close view of the beginning recoater region shows that the recoater moves every 30 seconds. This matches the frequency of the recoater commands on the machine script. The recoater spikes reach up to $0.2 \mathrm{~g}$.

39. A closeup plot of a single recoater movement in the beginning of the print contains an initial acceleration, movement, and then an abrupt halt as it hits two stoppers.

40. A closeup of the warpage section (b) of the print demonstrates the higher acceleration during the recoater passes than in the beginning recoater section (a)

41. As seen in the closeup of the later recoater section of the build, recoater spikes do not appear in the accelerometer profile because of the large amount of powder on the build plate. Instead, the altering high and low sections are due to other machine noise being damped differently based on the recoater position.

42. A closeup of the moment the machine is paused reveals a decrease in the magnitude of the accelerometer noise. The periodic behavior of the later recoater section is lost and random noise fills the accelerometer channel.

43. Fourier Transforms of the Machine On (a) to Machine Off (b) show the more prominent pattern in frequencies the Machine On. The Wavelet

Decomposition of Machine On (c) also has a prominent hum at $700 \mathrm{~Hz}$ that is missing from the Machine Off plot (d).

44. A close up of the accelerometer versus time plot during warpage. The warpage creates a variety of different recoater spikes that are inconsistent in amplitude. . 58

45. A closeup of the recoater movement in the warpage section of the build is characterized by large amplitudes when the recoater blade contacts sharp or severe warpage sections and noisy smaller spikes around the main spike as the recoater runs over lower warpage areas.

46. The acceleration versus time plot of the large recoater spike does not exhibit free vibrations implying that it is overdamped. This damping could be due to the position of the recoater blade on top of the build plate during the spike........ 60

47. The Wavelet Decomposition plot demonstrates that while the Warpage spike has a large magnitude, it cannot excite higher frequencies.

48. Three acceleration versus time plots indicate there were three potential crack events captured by accelerometer data during the cracked build 0056 . 
49. The cracks in the left leg (a) and the right leg (b) of the test coupon are viewed using a MicroVu.The crack in the right leg (b) is v shaped. This crack shape may be formed by two separate crack events.

50. A shift line seen in the photograph of the test coupon occurs when the test coupon moves during the build and the printer continues to print as if the test coupon has not moved.

51. A snapshot of a crack is distinguishable by its sudden large amplitude and its one-sided rate of decay.

52. Acceleration versus time plots of the Crack (a) and the Modal Hammer (c) shared similar profiles compared with the Recoater Movement (b).

53. An Acceleration versus time plot shows there is an average of five data points per peak, which is low but acceptable in signal analysis.

54. The frequency versus time plot of the three potential cracks had the same overall shape.

55. The frequency versus time plots of the crack (a) and the modal hammer test (c) both contained higher frequencies than the recoater movement (b)

56. The modal shape for $2744 \mathrm{~Hz}$ is shown in the Solidworks Simulation (a). The higher frequency of $5140 \mathrm{~Hz}$ produces a twisting mode shape of the parts (b).... 73

57. The camera's photos can pick up movements in the test coupon between layers. The green vector (right) shows the difference in test coupon leg placement from the previous layers photo (left).

58. A photograph of the experimental set up shows the iphone was placed on the outside of the build chamber door. It was not rigidly mounted.

59. The full audio file on $\mathrm{dB}$ versus time indicate it is difficult to distinguish between normal operation and warpage.

60. The recoater movements in this Decibel versus time plot of the audio data are not as easily identifiable as the accelerometer data plots.

61. Fourier Transform results indicate that low frequencies are predominant in the audio data. 


\section{Chapter 1: Introduction}

Additive manufacturing (AM), commonly known as 3D Printing, is revolutionizing manufacturing and product design in engineering. Parts with complex geometry can now be manufactured in less than a day with limited hours of manual labor. The general definition of AM is "taking virtual blueprints from computer aided design (CAD) or animation modeling software and "slicing" them into digital cross-sections for the machine to successively use during its controlled build process" (Society of Manufacturing Engineers). The current study focuses on metal AM, specifically Selective Laser Melting (SLM).

In SLM, metal powder is spread evenly in a layer. A laser then traces out the bottom cross section of the build object (part). Another layer of powder is placed on top and the process repeats until the entire part is built.

AM parts created using the SLM process are prone to defects created during the build process. These defects arise due to the high temperatures present when the laser melts the powder. Some common defects include cracking and warpage due to the part shrinking while cooling. Currently defects are discovered only after the part is removed from the machine. By detecting the defects in real time, a build's printing parameters could be adjusted to fix defects in situ, saving both time and money. 
The goal of this study was to determine a method to detect defects during the printing process. To accomplish this task, the project had several objectives:

- Fabricate a part that has inherent defects while collecting sensor data.

- Analyze sensor data to determine when the defect occurred.

- Characterize defect failure mode.

In the future, the deliverables from this project could be expanded into artificial intelligence and machine learning algorithms that would control the machine in real time and address defects as they arise, for example stopping the print in an event of a crack.

\subsection{Selective Laser Melting Process}

SLM is a powder bed fusion process. To fabricate a part, build material in powder form is spread evenly in a thin layer across a plate (Figure 1). A laser then traces out the bottom cross section of the part. Powder in the laser's path is melted together while the rest remains in powder form. The plate is then lowered, and another layer of powder is added. The powder spreading (recoating) and laser melting process continues until the part is complete. After many layers, the finished part lies buried in powder. The process of creating a part is called a build or a print. 
(a)

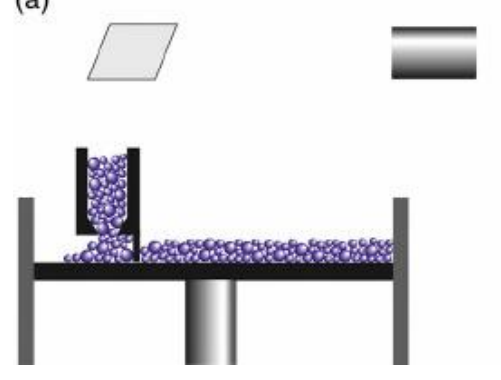

(b)

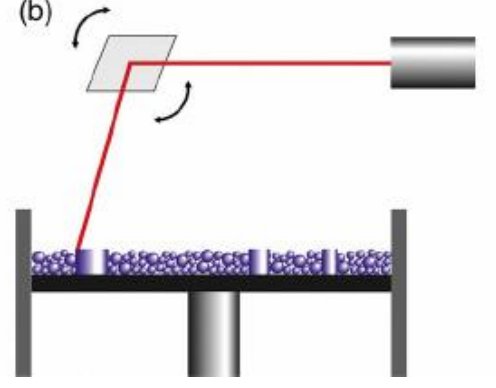

(c)

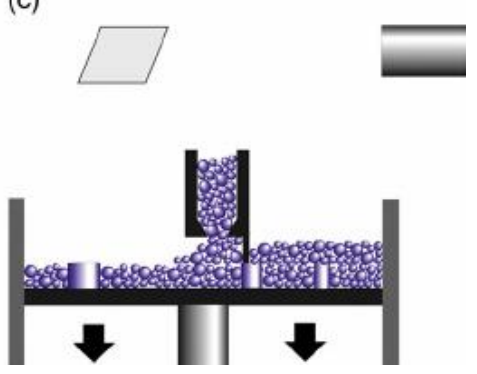

Figure 1. A diagram of the SLM printing process. Metal powder is spread in a thin layer across a metal plate (a). A laser the traces out the cross section of the part $(b)$. The plate is then lowered to the thickness of a layer of powder and the process repeats (c) (Cai, Malcolm and Wong).

\subsection{SLM Solutions 125 Hardware Overview}

The study utilized Cal Poly's SLM Solutions 125 HL (SLM Solutions) (Figure 2). The machine was donated by Lawrence Livermore National Laboratory in 2017. It is primarily used for senior projects and masters theses. SLM Solutions is a German company and one of the leading industrial solutions for metal additive manufacturing. EOS and Concept Laser are the two other large metal additive manufacturing companies. In this report, the machines produced by these companies will be referred to by the company name as that is what the machines are officially titled.

The SLM Solutions machine has four main components: build chamber, powder system, laser system, and computer controls. The build chamber is where the laser, powder, and argon interact to produce a well-formed part. The powder system brings the powder from the inlet container on the top of the machine to the build chamber. This system also takes unmelted powder to the overflow containers to be reused. The laser system involves the laser generation, as well as the path the laser takes to get to the build chamber, including 
a set of mirrors. The computer control uses Windows 7 as a user interface. From the computer, the operator can turn on and turn off different features of the machine as well as set up and monitor the print, also called a build.

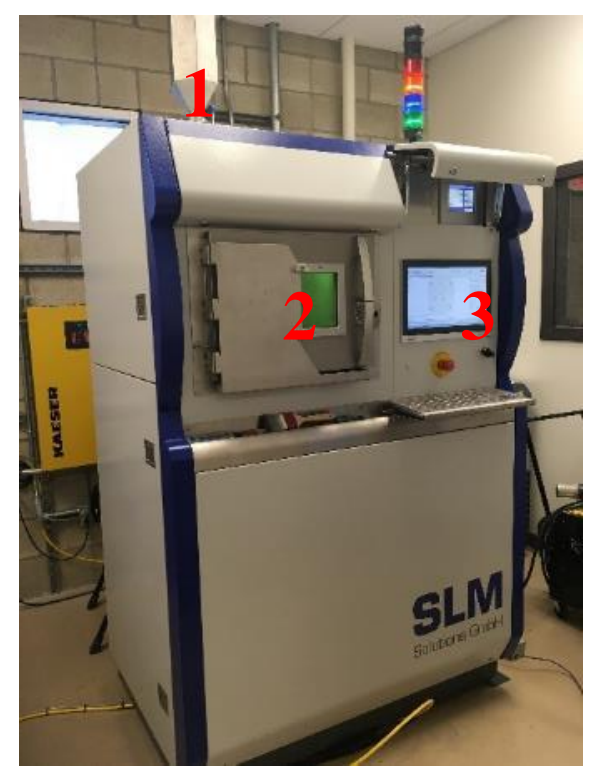

Figure 2. Photograph of the SLM Solutions 125 HL. The printer has a build volume of $125 \times 125 \times 125 \mathrm{~mm}^{3}$. The machine includes powder inlet (1), build chamber (2), and the computer (3).

Cal Poly's SLM Sieving Station, PSM 100, is used for powder processing between builds (Figure 3). The unmelted powder used in a build is filtered so it can be reused in the next print. In the powder bed, the area next to the laser path is a heat affected zone. Powder particles in this area often half melt together, creating large irregular shaped particles that can affect the quality of a part (Figure 4). To avoid printing with non-uniform powders, the sieving station removes abnormal particles. 


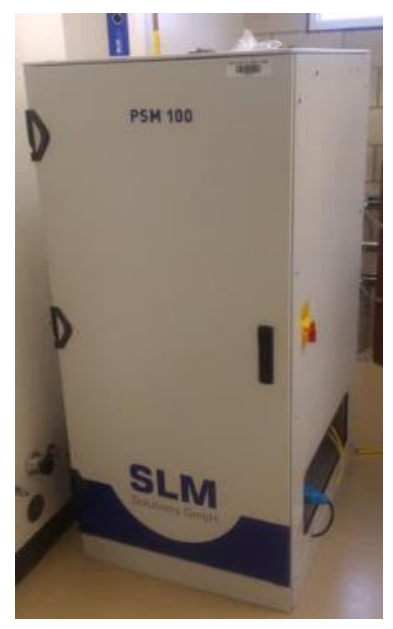

Figure 3. Photograph of the SLM Sieving Station that removes large irregular particles from the powder so the powder can be reused in the next build.

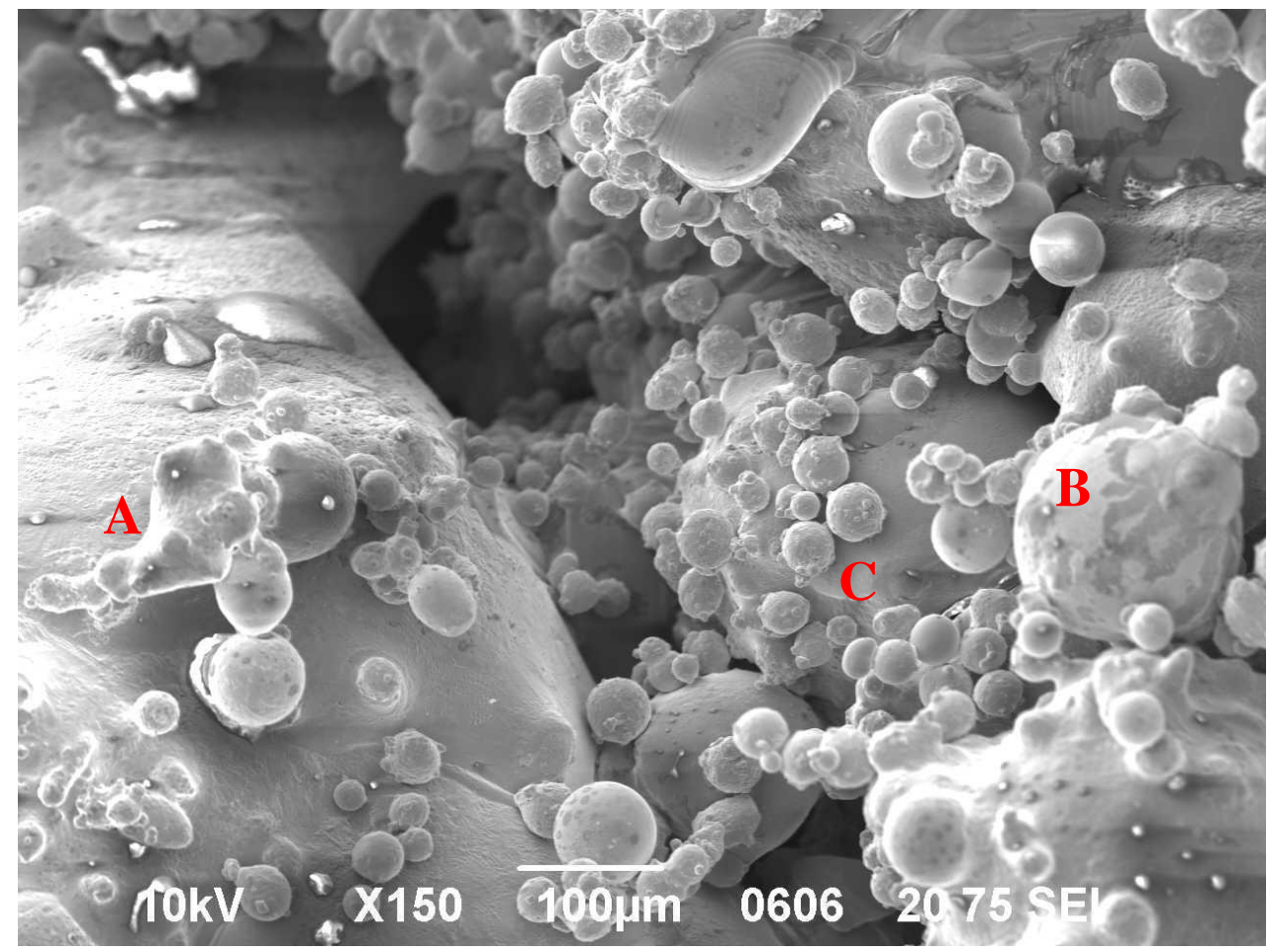

Figure 4. A SEM photo reveals irregular conglomerates of particles (A) are created when they are heated enough to partially fuse together. Heat affected particles $(B)$ occur when the particle is melted multiple times. Normal Particles $(C)$ are separate and free flowing. 


\subsubsection{Build Chamber}

The build chamber is the manufacturing location in the SLM Solutions machine. The build chamber contains the hopper, recoater, and build plate (Figure 5). Before the build begins, the chamber is sealed and filled with ultra-high purity Argon. The chamber attempts to hold the atmosphere at $0 \%$ oxygen and will pause the build if the level reaches 2\%. Inert gases such as Argon are used in the SLM process as a welding shielding gas to move away ash and prevent oxidization. In the floor of the build chamber is the build plate where the part is built. Powder is spread across the build plate using the powder recoater, which refills at the powder hopper in the back of the chamber.

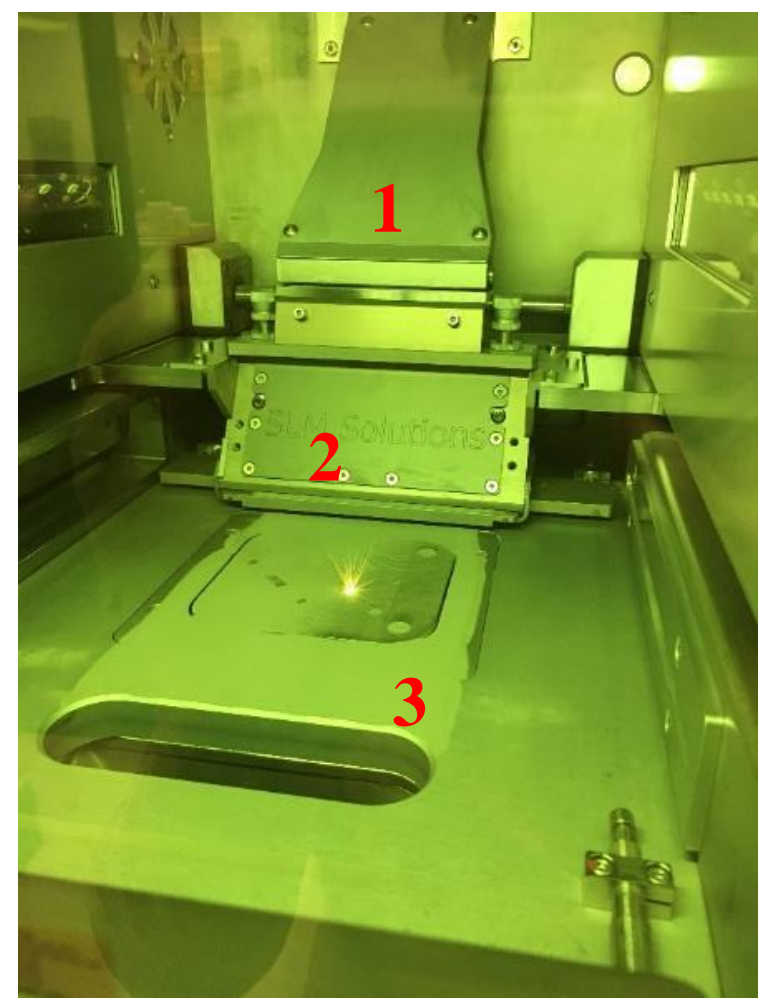

Figure 5. As seen in the photo taken through the build chamber window, the build chamber contains (1) the powder hopper, (2) the recoater, (3) the build plate. 
The build volume for this model is $125 \times 125 \times 125 \mathrm{~mm}^{3}$. This means that the build plate is a $125 \mathrm{~mm}$ square and can move down $125 \mathrm{~mm}$ below the floor of the build chamber. As each layer of powder is added, the build plate lowers through a cavity in the floor of the build chamber using a leadscrew. Beneath the build plate is a heating pad supported by the build platform. The operator can set the temperature of the build plate up to 200 ${ }^{\circ} \mathrm{C}$. The temperature of the build plate, build chamber, and other key cabinets in the printer are monitored and recorded during each build.

The recoater includes a rubber blade to spread the powder and a hopper to store powder (Figure 6). Unique to the SLM Solutions machine, the recoater is filled with powder in the back of the chamber at the powder hopper. It then deposits powder across the build chamber. The recoater waits at the front of the chamber as layer 1 is welded with the laser. Once layer 1 is complete, the recoater spreads powder from front to back (Figure 7) then stays at the back of the chamber as layer 2 is melted. The recoater then refills and spreads powder back to front for layer 3 .

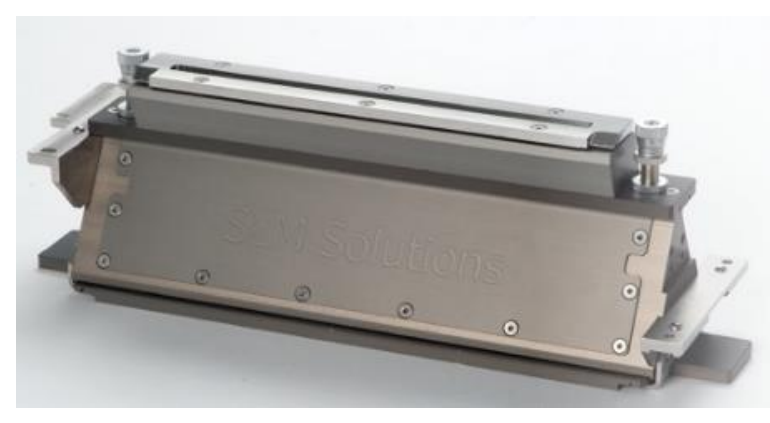

Figure 6. CAD model of a SLM Solutions recoater. The recoater has a twin rubber blade to allow for even spreading of powder in both directions across the build. The recoater can hold two layers of powder, allowing it only to refill at the hopper at the back of the machine. (SLM Solutions Group) 


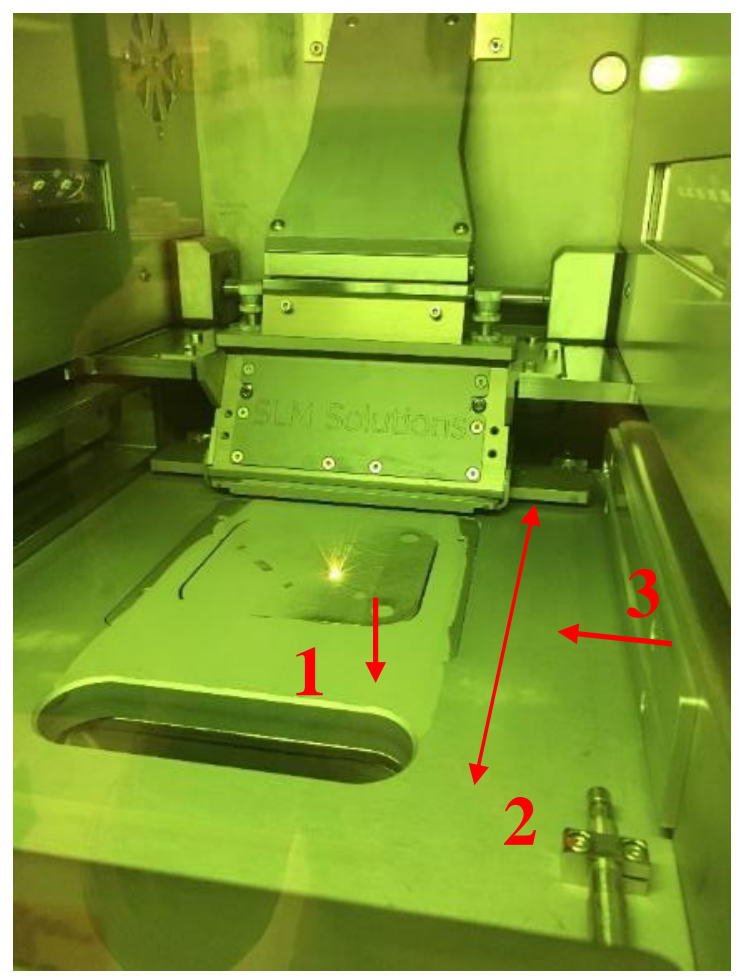

Figure 7. The labeled photo of the inside of the build chamber shows that the build plate lowers down as each layer is added to the part (1). The recoater blade moves from front to back of the machine to add new layer of powder (2). The Argon flows from right to left to maintain the atmosphere and move ash away from the part (3).

\subsubsection{Powder}

The powder is stainless steel 316L purchased from SLM Solutions. The powder hopper container holds approximately $22 \mathrm{~kg}$ of powder. After each build, unmelted powder is collected and sieved in the Sieving Station then reused for the next build. When the total powder quantity is reduced to approximately $10 \mathrm{~kg}$ of powder, new powder is added. Due to the recycling process, the powder used in current prints contains some of the original powder from the first build a year ago. Although the powder is filtered in the sieving station, not all ash and smaller irregular particles are removed. The presence of irregular particles may result in uneven powder distribution across the build plate. 


\subsubsection{Laser}

The laser is projected from the top of the build chamber through a window onto the powder layer. The maximum laser power is $400 \mathrm{~W}$; however, the exact parameters used are determined by each part build file loaded into the machine. The various settings, or print parameters, are pre-determined by the engineer for each build individually (Table 1). Routine builds often reuse the same parameter sets, while complicated builds undergo an iterative process to determine the optimal parameter settings. Print parameters are further described in Chapter 2.

Table 1. There are various print parameters that determine the quality of the build. Some of the parameters are listed below with their units. These parameters are further defined in Chapter 2.

\begin{tabular}{|l|l|}
\hline Parameter & Units \\
\hline Support Structure & - \\
\hline Argon Pump Speed & $\%$ \\
\hline Minimum Scanning Time & $\mathrm{sec}$ \\
\hline Layer Height & $\mathrm{mm}$ \\
\hline Build Plate Heater & ${ }^{\circ} \mathrm{C}$ \\
\hline Laser Power & watts \\
\hline Scan Speed & $\mathrm{mm} / \mathrm{s}$ \\
\hline Fill Pattern & $\mathrm{Chess,} \mathrm{stripe}$ \\
\hline Stripe Size & $\mathrm{mm}$ \\
\hline Hatching Distance & $\mathrm{mm}$ \\
\hline
\end{tabular}




\subsubsection{Camera}

Above the build chamber there is a camera. The camera is used by the SLM Solutions software to analyze the powder coverage across the build plate. A photograph is taken after each movement of the recoater and before the laser begins. If the powder spread across the build plate is insufficient, the recoater will recoat again. If the powder spread has not been fixed in an operator set number of attempts, the build is paused. The machine script documents all actions given by the computer to various hardware in the machine. All photos along with the machine script are saved after each build.

\section{$\underline{1.3 \text { Accelerometers }}$}

Two accelerometers - 1 Kistler Triaxial and 1 Kistler uniaxial - are attached at the underside of the heated pad. These sensors were added in 2017 as part of a Cal Poly Senior Project (Coria, Whipple and Grant). The cables from the accelerometers are brought up to the top of the machine through pre-existing space around the powder hopper (Figure 8). The accelerometers are connected to the Kistler LabAmp DAQ which is then connected to a laptop through an ethernet cable. During experimental builds, called test coupons, the accelerometers were set to collect data at 12500 samples per second $(\mathrm{Hz})$. 

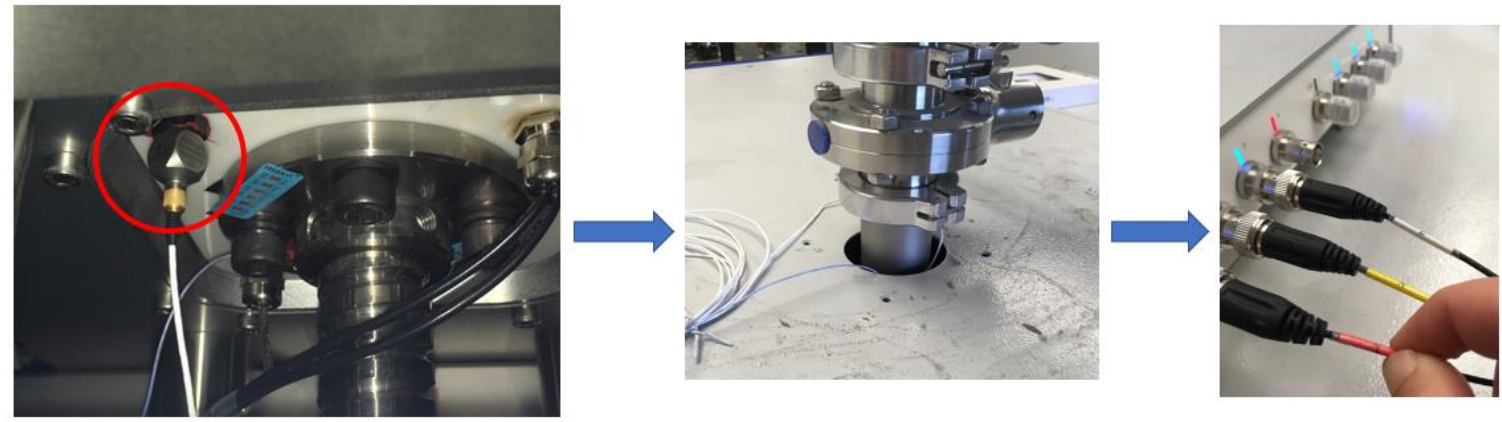

Figure 8. The diagram of the accelerometer connections shows the accelerometers are attached to the bottom of the build platform using silicone adhesive. The cables exit the

SLM Solutions machine through a hole in the top of the machine for the inlet of the powder. The cables are then connected to the DAQ.

\subsection{Defects in Selective Laser Melting}

In additive manufacturing, there are many types of defects unique to the process. Some of the common defects are:

- Warpage - Edges of the part peel up due to shrinkage while cooling. This can lead to damage of the recoater and effect the powder spread in future layers.

- Cracks - When a large part cools, the shrinkage is sometimes so great that it causes lower portions of the part to crack so that it can shrink. This defect is the focus of the current work in defect detection.

- Trapped Powder - While AM can create hollow parts, the powder bed fusion process requires the hollow parts have a hole allowing unmelted powder inside to drain completely out.

- Discoloration - The part may be brown or ashy in color. This occurs when ash is introduced into the melt pool. This can be caused from (1) the flow of Argon stopping due to a pause in the build, (2) the flow of Argon not being strong enough to completely blow away the ash. 
Defects can cause issues both during the printing process as well as when the parts are implemented into their final assemblies. For example, warping causes the part to curl up during the build and tear the rubber recoater blade. The torn blade then spreads the powder unevenly, causing some sections of the build to be thicker than others. Thick areas are problematic because they do not melt as hot as thin powder areas and can result in incomplete melting of powder. In addition, the thick areas act as a dam, causing poor powder flow to areas behind them (Figure 9). After the part is removed from the printer, warpage can be a further issue because the part does not match the intended geometry.

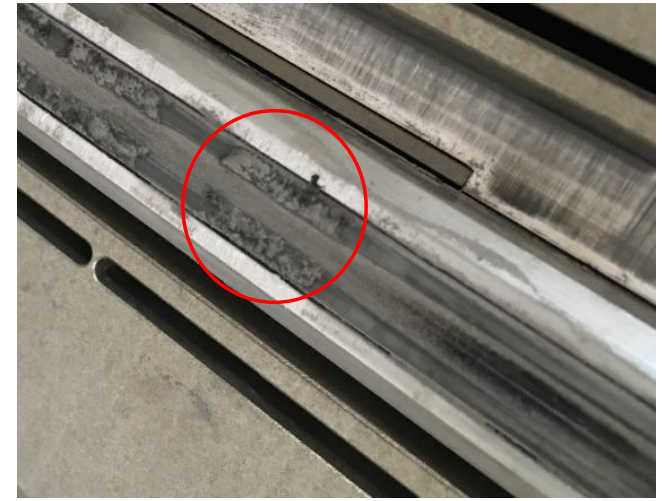

(a)

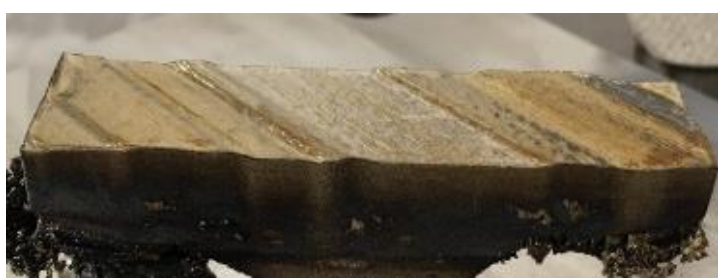

(b)

Figure 9. These photos demonstrate warpage can negatively impact later layers of the part through destruction to the recoater blade. The pieces of rubber missing from the recoater blade (a) lead to unevenness in powder spread. Thicker layers in the powder spread translate to thicker regions in the build layers. These thicker regions produce nonuniform part geometry $(b)$ and material properties.

Unlike other manufacturing processes, detecting defects as they occur is problematic because the part is not visible to the operator. During the powder bed fusion process, the lower sections of the part are sunken in the build chamber and covered by metal powder. 
If the defects can be seen on the current cross section of the part, as is the case with extreme warpage, the build can be stopped manually; however, in the case of cracking, the crack occurs in areas below the current cross section which will be unnoticed until the part is removed at the end of the build.

Commercial technology to monitor print quality in situ is limited. SLM Solutions provides a built-in photo analysis software to monitor powder spread coverage across the build plate. In 2017, SLM Solutions released a quality software package that monitors melt pool radiation, powder layer uniformity, and laser power. While this software package is useful in adjusting print properties as the build is progressing, it does not monitor for defects that occur beneath the top powder surface, such as cracking.

\subsection{Analysis Methods}

Three types of data were collected during each test coupon's build: acceleration, image, and audio. The purpose of the data was to determine when a defect, either warpage or cracking, occurred during the build. The acceleration data was taken with the installed accelerometers, the image data used the SLM Solutions machine's built in camera, and the audio data was using an iphone placed outside of the machine. The accelerometer and audio data were analyzed using Fourier Transform, Wavelet Transformation, and Amplitude Filters in Matlab. The image data used the image toolbox in Matlab to analyze differences between sequential images. The analysis of the data collected is detailed in Chapter 4. 


\section{$\underline{1.6 \text { Research Overview }}$}

The scope of the project was to record a crack event using an accelerometer to determine if an accelerometer can detect defects in the SLM process. The first phase of the research, discussed in Chapter 2, was to develop print parameters to create the intended crack defect in the experimental part called the test coupon. After a crack was produced in a test coupon, the failure was characterized as seen in Chapter 3. Lastly, accelerometer data was analyzed to determine when the failures occurred as discussed in Chapter 4. 


\section{Chapter 2: Inducing Part Failure}

To detect a defect in a SLM Solutions part, a test coupon first needed to be printed that contained that defect. The test coupons printed in this study contained two types of defects. The first was warpage during the printing of the legs of the part (Figure 10, left). The other defect was cracking in the legs of the parts later in the build after they were printed (Figure 10, right). The crack would occur due to heat differential between the top of the part and the build plate. The original goal of the project was to observe cracking. This proved difficult, however, because a heat differential also causes warpage, which often destroyed the test coupons before cracking could occur. Warpage in the upper portion of the leg emerged above the top powder layer and caught on the recoater blade. After several layers of bending the leg, the recoater blade eventually tore the leg at the intended cracking point, detaching the upper leg from the lower leg.
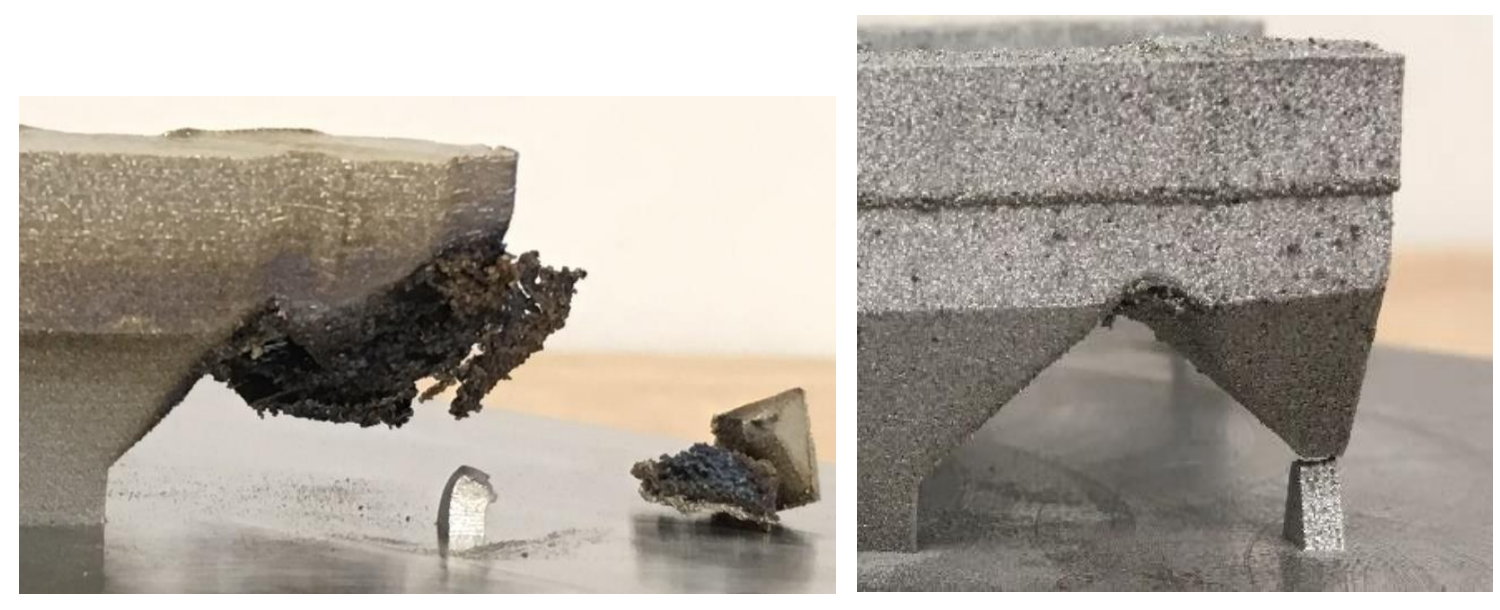

Figure 10. The two defects produced in the test coupons were (left) warpage and (right) cracking as seen in the photos. Warpage features the remains of the lower leg with the upper leg pulled off. The upper leg was pulled off by the recoater hitting into its warpage. The burning on the top are of the leg is due to the laser melting loose powder. The loose powder is pushed by the recoater as it is not attached rigidly. This results in the burning appearance of the test coupon. 
The following section will first discuss the development of the test coupon design provided by Lawrence Livermore National Laboratory. The development and results of two print parameter sets that were used to print the test coupons at Cal Poly are then discussed.

\section{$\underline{2.1 \text { Test Coupon Design }}$}

The first phase of the project was to print an AM part that would predictably fail. For the study, a design provided by Lawrence Livermore National Laboratory (LLNL) was chosen (Figure 11).

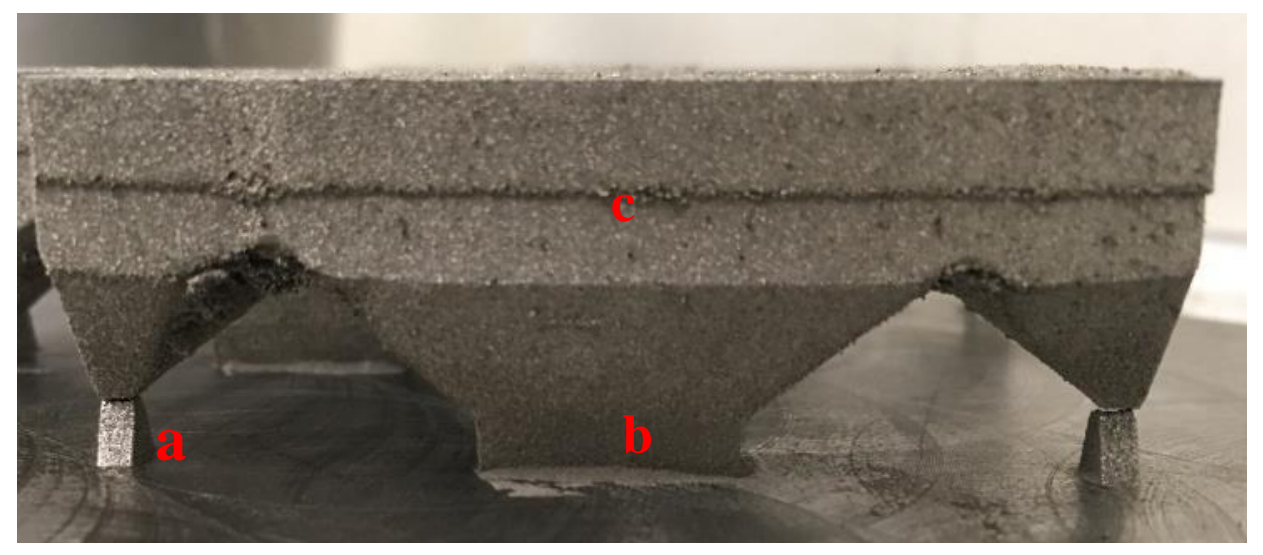

Figure 11. A photograph of the experimental part contains two legs with weak cracking locations (a), a middle support leg (b), and an upper high heat portion (c).

The test coupon includes three legs. The two outer legs are hour glass shaped. The small cross section is designed to be prone to cracking under any loading condition (shear, bending, or tensile). The middle leg acts as a support stand. The top section of the test coupon is the heat reservoir. The top section's large mass means it should retain heat for an extended time after it is built. The temperature differential between the top of the test 
coupon and the build plate is designed to stress the small cross sections in the legs and cause them to crack as the upper mass cools and shrinks.

The test coupon was chosen for the project because it had cracked cleanly when printed at LLNL using a Concept Laser machine. Differences between the print parameters and the hardware on LLNL's Concept Laser and Cal Poly's SLM Solutions machines, however, brought challenges in replicating the same failure mode predictably at Cal Poly. The first five builds used SLM Solutions' default stripe laser pattern, while the second five builds more closely replicated LLNL's efforts by using a chess laser pattern.

\subsection{Print Parameters Development}

Print parameters on the SLM Solutions machine are specified by the operator in the Materialise Magics build processor software. Each part on a build can have its own print parameters. The SLM Solutions build processor user manual was referenced when designing print parameters for each build (Table 2) (Materialise). Some of the common terminology used in print parameter options are described in Figure 12. 
Table 2. A summary of print parameter definitions, some are visually defined below.

\begin{tabular}{|c|c|}
\hline Parameter & Definition \\
\hline Support Structure & $\begin{array}{l}\text { Support structure is added between the part and the build plate } \\
\text { to reduce movement/instability during the print and move heat } \\
\text { away from the melt pool. None of the prints in this experiment } \\
\text { used support structure. }\end{array}$ \\
\hline Argon Pump Speed & $\begin{array}{l}\text { The speed of the vacuum pump determines the flow rate of the } \\
\text { Argon across the build chamber. This is maintained at } 68 \% \text { for } \\
\text { all builds. }\end{array}$ \\
\hline $\begin{array}{c}\text { Minimum Scanning } \\
\text { Time }\end{array}$ & $\begin{array}{l}\text { The minimum amount of time between recoater movements. If } \\
\text { the laser's scanning takes less than the minimum scan time, the } \\
\text { build will pause until the minimum scanning time is elapsed. }\end{array}$ \\
\hline Layer Height & The thickness of each powder spread. \\
\hline Build Plate Heater & $\begin{array}{l}\text { The temperature of the build plate is set from } 0-200{ }^{\circ} \mathrm{C} \text {. Most } \\
\text { parts are printed with the build plate set to } 150^{\circ} \mathrm{C} \text {. The test } \\
\text { coupons in this study were printed with the heater off. }\end{array}$ \\
\hline Power & The laser power. \\
\hline Scan Speed & The laser scan speed. \\
\hline Fill Pattern & The pattern used for hatching (Chess, stripe, etc). \\
\hline Stripe Size & The length of one laser pass. \\
\hline Hatching Distance & The distance between laser scan stripes. \\
\hline Border & The region around the outside of the cross section. \\
\hline Outer Hull & $\begin{array}{l}\text { The main inside region of the cross section. The inside region } \\
\text { can further be divided into the core and the outer hull if the } \\
\text { hatching pattern requires it. }\end{array}$ \\
\hline Fill Contours & The region between the border and the outer hull. \\
\hline Transition Contour & The region between the border and the outer hull. \\
\hline Up-Skin & $\begin{array}{l}\text { Surfaces within } 45 \text { degrees of horizontal and will be visible } \\
\text { once the part is complete. Enabling up-skin means the laser will } \\
\text { scan the surface with a higher laser power to produce a better } \\
\text { surface finish. }\end{array}$ \\
\hline
\end{tabular}




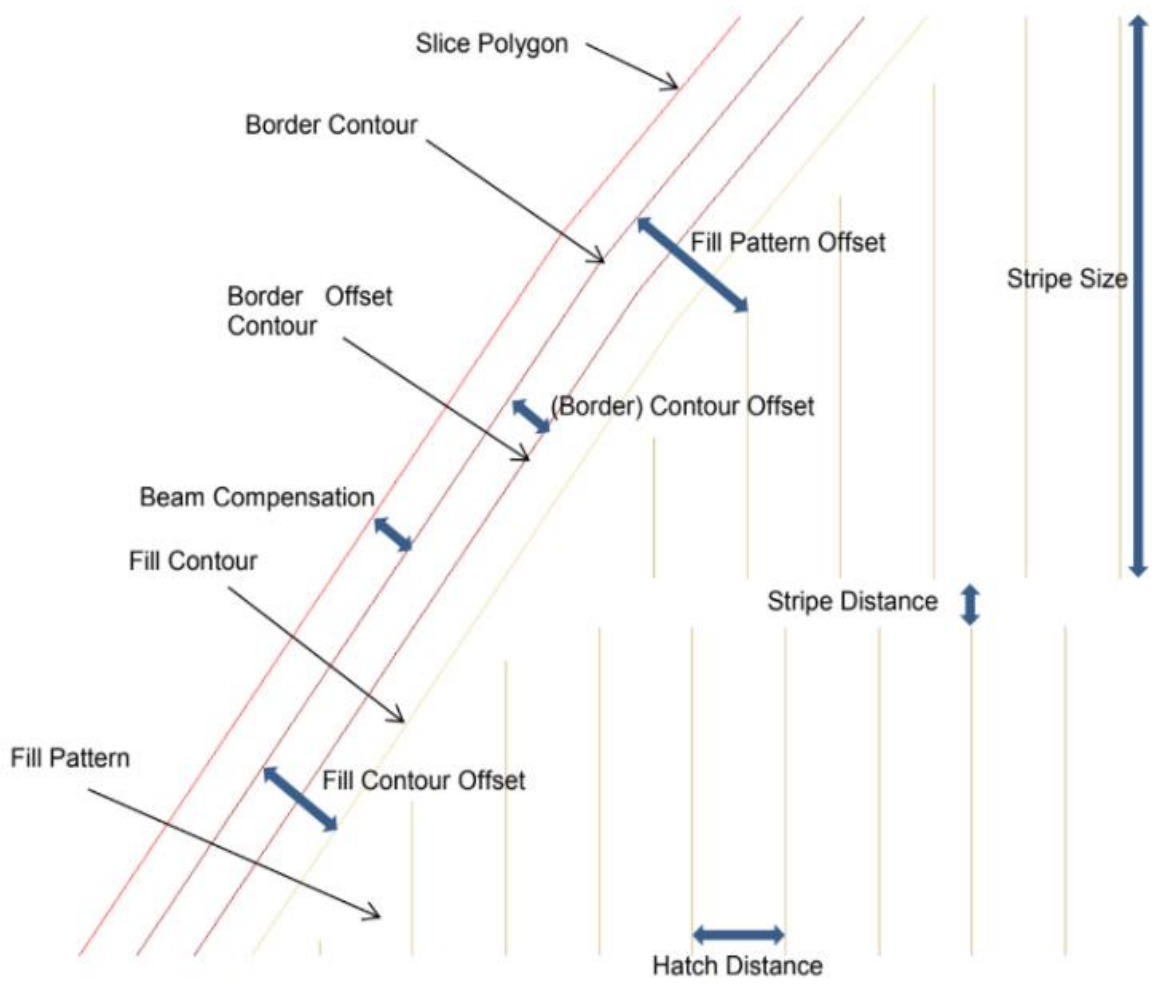

Figure 12. Common Materialise Magics Print Parameter Terminology. (Materialise)

The test coupons were printed with two different print parameters, each named after their hatching fill pattern. The first print parameters are called the stripe parameters. The stripe parameters are the default parameters on the SLM Solutions machine. The second set of parameters are called the chess parameters and were developed after severe warpage was encountered when printing the test coupons with the stripe parameter set. The chess print parameters are modeled after LLNL's print parameters on the Concept Laser machine where the test coupon was cracked originally. 


\subsubsection{Stripe Parameters}

In the stripe hatch pattern, the laser moves in lines across the entire cross section of the part (Figure 13).

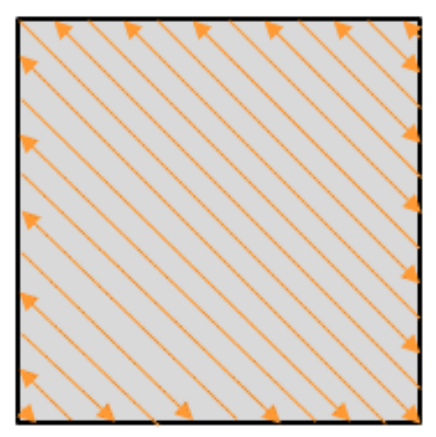

Figure 13. A stripe hatch pattern means the laser crosses the entire width of the part before beginning the next stripe. (Rensihaw)

The stripe parameter file used was the default SLM Solutions print parameter setting. This setting was not optimized specifically for the Cal Poly SLM Solutions $125 \mathrm{HL}$ machine. While most SLM Solutions 125 HL builds are good quality when printed using this setting, optimizing the parameters could lead to a higher print quality in all parts printed by the Cal Poly SLM Solutions machine. In Table 3 are fundamental stripe parameter settings. The test coupon's difficult geometry caused severe warpage when using the stripe print parameters. The chess print parameters were developed after several test coupons did not print as desired when using the stripe parameters. The chess and stripe hatching patterns are options available on the SLM Solutions machine. The combination of different parameter settings creates a unique print parameter set. 
Table 3. Key Stripe Print Parameters.

\begin{tabular}{|l|l|l|}
\hline Parameter & Value & Units \\
\hline Minimum Scanning Time & 15 & $\mathrm{Sec}$ \\
\hline Layer Height & 0.03 & $\mathrm{Mm}$ \\
\hline Build Plate Heater & Off & - \\
\hline Hatching Up-Skin & On & - \\
\hline Hatching Border Distance & 0.18 & $\mathrm{Mm}$ \\
\hline Border Power & 100 & $\mathrm{watts}$ \\
\hline Border Scan Speed & 250 & $\mathrm{~mm} / \mathrm{s}$ \\
\hline Fill Contours Distance & 0.08 & $\mathrm{Mm}$ \\
\hline Number of Fill Contours & 1 & - \\
\hline Hatching Outer Hull Distance & 0.12 & $\mathrm{Mm}$ \\
\hline Outer Hull Scan Power & 200 & $\mathrm{Watts}$ \\
\hline Outer Hull Scan Speed & 800 & $\mathrm{~mm} / \mathrm{s}$ \\
\hline Fill Pattern & Stripes & - \\
\hline Stripe Size & 10 & $\mathrm{Mm}$ \\
\hline Transition Contour Distance & 1 & $\mathrm{Mm}$ \\
\hline $\begin{array}{l}\text { Number of Transition } \\
\text { Contours }\end{array}$ & 1 & - \\
\hline
\end{tabular}

\subsubsection{Chess Parameters}

After discussing with the LLNL operator about the print parameters used for the original LLNL build that printed past warpage, significant differences from the SLM Solutions default stripe parameters were discovered. The new parameters developed from this discussion with LLNL were used in the second half of the Cal Poly builds and are labeled chess after the hatching pattern used (Figure 14). 


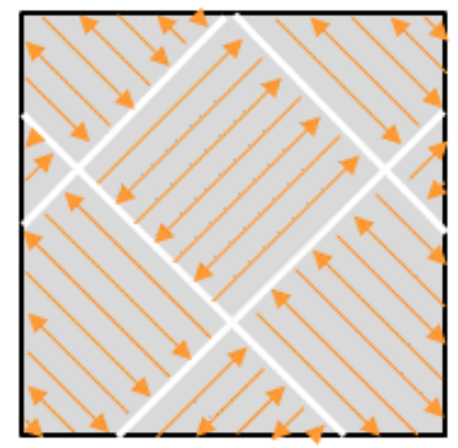

Figure 14. A diagram of the chess pattern consists of squares of stripe hatches. These squares are traced out by the laser in a random pattern to better distribute the heat. (Rensihaw)

Overall, the LLNL parameters lead to a better heat distribution in the powder bed. This made the LLNL build more likely to print past warpage than the test coupons created on the SLM Solutions machine using the stripe parameter settings. The differences between the default stripe parameter settings on the SLM Solutions and the settings used on the Concept Laser machine at LLNL are outlined in Table 4. 
Table 4. Differences between Concept Laser print parameters at LLNL and SLM Solutions' default stripe parameters used in the first builds at Cal Poly.

\begin{tabular}{|l|l|}
\hline $\begin{array}{c}\text { Concept Laser's } \\
\text { Difference }\end{array}$ & \multicolumn{1}{c|}{ Impact on Build } \\
\hline $\begin{array}{l}\text { Smaller Build plate } \\
\text { dimensions }\end{array}$ & $\begin{array}{l}\text { More heat in lower portion of powder bed. SLM Solutions' } \\
\text { larger build plate }\left(125 \mathrm{x} 125 \mathrm{x} 25 \mathrm{~mm}^{3}\right) \text { acts as a heat sink } \\
\text { at the bottom of the part, only reaching } 50^{\circ} \mathrm{C} \text { during a test } \\
\text { coupon build. }\end{array}$ \\
\hline $\begin{array}{l}3 \text { parts instead of 1 } \\
\text { part per build }\end{array}$ & $\begin{array}{l}\text { Increased laser time allows for heat flow away from melt } \\
\text { pool into rest of powder bed. More parts also mean more } \\
\text { heat is distributed into more areas in the powder bed. }\end{array}$ \\
\hline $\begin{array}{l}1 \text { direction } \\
\text { recoating }\end{array}$ & $\begin{array}{l}\text { The Concept Laser machine can only coat in 1 direction, } \\
\text { meaning it must return on each recoat - effectively } \\
\text { increasing the time between layers. }\end{array}$ \\
\hline $\begin{array}{l}\text { 40\% reduction of } \\
\text { energy per unit } \\
\text { length of laser scan }\end{array}$ & $\begin{array}{l}\text { The Concept Laser machine had lower laser power and } \\
\text { speed, resulting in energy per unit length of 0.15 J/mm } \\
\text { instead of the stripe parameters' 0.25 J/mm. Less energy } \\
\text { will melt the powder in a more controlled manner and may } \\
\text { reduce the heat affected zone around the print. }\end{array}$ \\
\hline $\begin{array}{l}\text { Lack of additional } \\
\text { laser passes on key } \\
\text { regions of the build }\end{array}$ & $\begin{array}{l}\text { The Concept Laser machine lacks the border and up-skin } \\
\text { features that were enabled on the stripe parameters on the } \\
\text { SLM Solutions. These extra passes in these regions } \\
\text { increase the amount of heat into warpage prone areas. }\end{array}$ \\
\hline $\begin{array}{l}\text { Chess hatching } \\
\text { pattern }\end{array}$ & $\begin{array}{l}\text { LLNL used a } 5 \text { mm chess pattern instead of a stripe } \\
\text { pattern. The random scanning of the squares in the chess } \\
\text { pattern spread heat more evenly through the build. In } \\
\text { addition, the increased number of overlapping boundaries } \\
\text { between the different squares results an anisotropic grain } \\
\text { structure in the metal that effects the cracking patterns at } \\
\text { the microscopic level (Carter, Martin and Withers). }\end{array}$ \\
\hline
\end{tabular}


To better replicate the LLNL build, a few changes were made from the default stripe print parameters to the chess print parameters. Important differences include:

- Increasing the number of parts from 1 to 3 in each build

- Reducing laser power and speed

- Increasing minimum scanning time

- Changing the fill pattern to $5 \mathrm{~mm}$ chess instead of stripes (Figure 15)

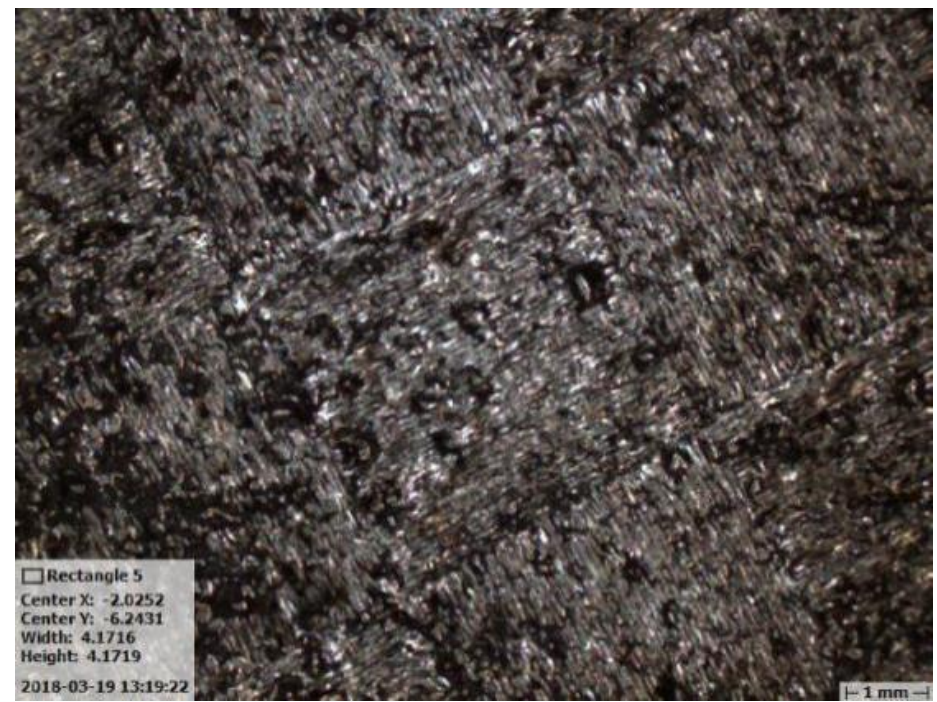

Figure 15. The chess hatch pattern is seen in a microscope image of the part surface.

The new chess parameters used on the SLM Solutions machine for the second half of the test coupons are summarized in Table 5. While the new chess print parameters were better suited for printing past the warpage in the leg, all the builds in the study displayed some warpage in the lower portion. 
Table 5. Important chess print parameters used when printing the test coupons are summarized alongside the default stripe parameter settings.

\begin{tabular}{|l|l|l|l|}
\hline Parameter & Chess & Stripe & Units \\
\hline Minimum Scanning Time & 30 & 15 & $\mathrm{sec}$ \\
\hline Layer Height & 0.03 & 0.03 & $\mathrm{~mm}$ \\
\hline Build Plate Heater & Off & Off & - \\
\hline Hatching Up-Skin & Off & On & - \\
\hline Hatching Border Distance & 0.09 & 0.18 & $\mathrm{~mm}$ \\
\hline Border Power & 60 & 100 & watts \\
\hline Border Speed & 600 & 250 & $\mathrm{~mm} / \mathrm{s}$ \\
\hline Fill Contours & Off & On & - \\
\hline Fill Contours Distance & - & 0.08 & $\mathrm{~mm}$ \\
\hline Number of Fill Contours & - & 1 & - \\
\hline Hatching Outer Hull Distance & 0.084 & 0.12 & $\mathrm{~mm}$ \\
\hline Outer Hull Power & 90 & 200 & Watts \\
\hline Outer Hull Speed & 600 & 800 & $\mathrm{~mm} / \mathrm{s}$ \\
\hline Fill Pattern & Chess & Stripes & - \\
\hline Field Size/Stripe Size & 5 & 10 & $\mathrm{~mm}$ \\
\hline Transition Contours & Off & On & \\
\hline Transition Contour Distance & - & 1 & $\mathrm{~mm}$ \\
\hline Number of Transition Contours & - & 1 & - \\
\hline
\end{tabular}




\section{Chapter 3: Part Failure Analysis}

This chapter will examine the physical defects in the ten test coupons built during the study. The first five builds used the stripe print parameters and the remaining five builds used the chess print parameters. All the builds had warpage. Only one build was able to print past the warpage to experience a crack.

\subsection{Warpage in Narrowly Supported Cross Sections}

Warpage was seen in all ten of the test coupon builds in the study. It is also a common defect in SLM parts in general. Warpage is caused by the shrinking of layers as they cool. The melt pool temperature is $1300{ }^{\circ} \mathrm{C}$. As the layer cools to $25^{\circ} \mathrm{C}$, room temperature, the metal can shrink up to $2 \%$ (Engineering ToolBox). For example, a part 3 inches long can shrink up to $0.06 \mathrm{in}$. This is significant as the part is rigidly connected to the build plate on the bottom layer. In long skinny flat parts, the warpage can be so severe that the build crashes: the part collides severely with the recoater and gets wedged underneath, preventing the recoater from moving, thus halting the build.

When printing the upper portion of the leg, its corners began to warp (Figure 16). Corners tend to warp because the heat from the laser repeatedly hits the same powder. The heat affected area is wider than the laser, so sharp geometry such as corners and edges are melted multiple times and begin to warp. Eventually, the warped corners were high enough above the powder layer that they caught and ripped the recoater blade (Figure 17). The torn recoater blade then resulted in an uneven distribution of powder across the bed. The uneven print surface is reflected in the resulting grain boundaries and surface of the test coupon (Figure 18). 


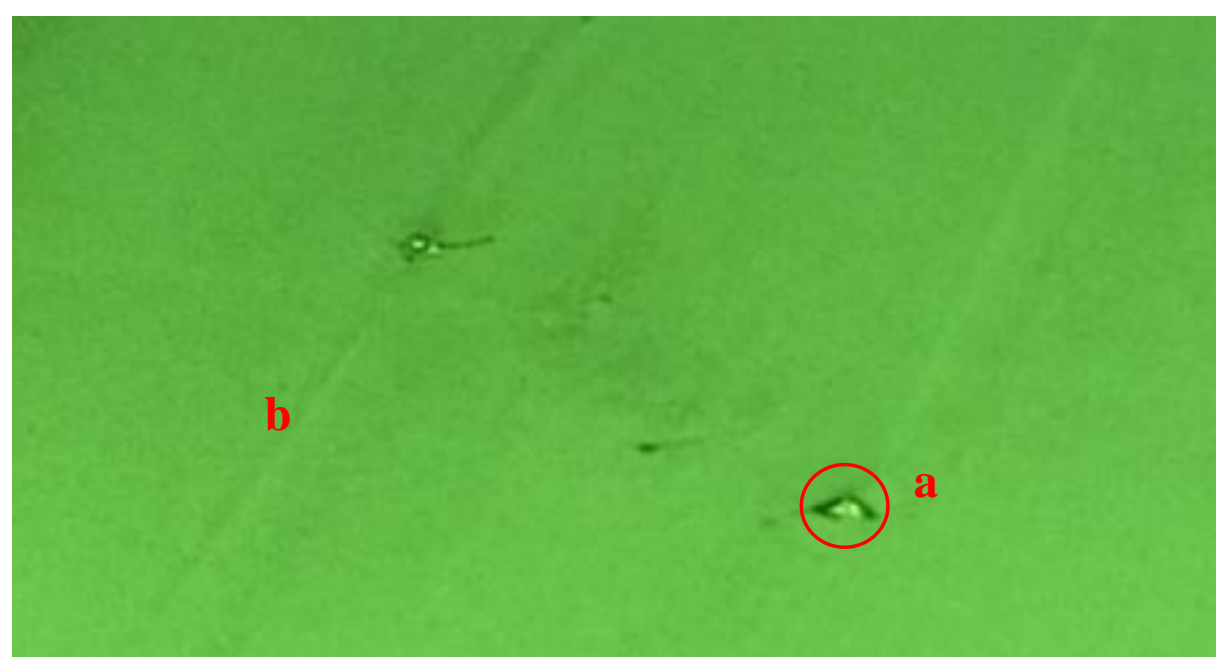

Figure 16. Photograph of the surface of the powder layer during warpage showing the corners of the leg warped upward (a). The warpage had previously ripped the recoater blade, resulting in uneven powder spread in subsequent layers as seen by the lines in the powder $(b)$.

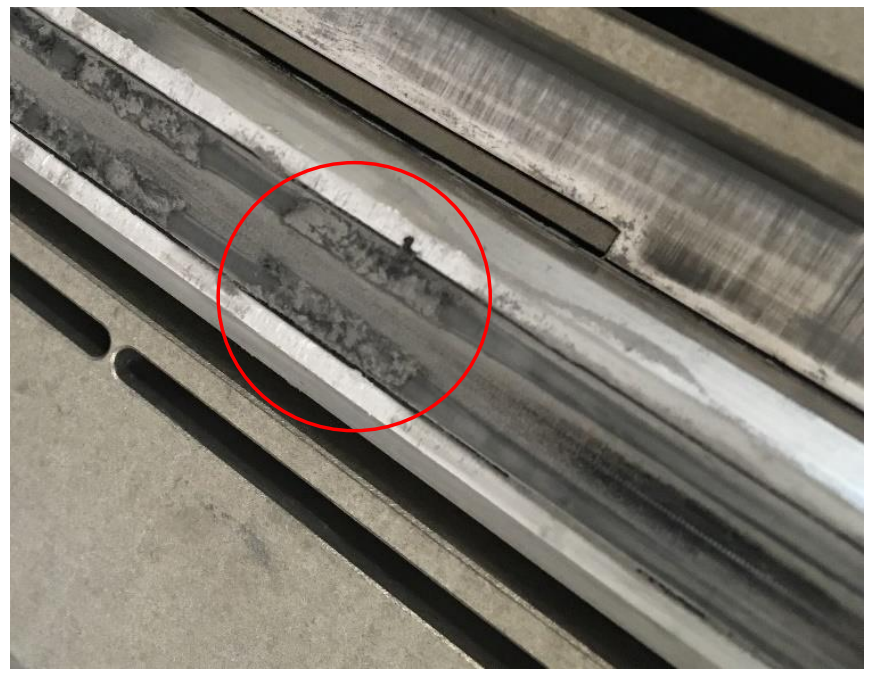

Figure 17. Photograph of a recoater blade damaged from hitting against sharp upturned corners of warpage. 


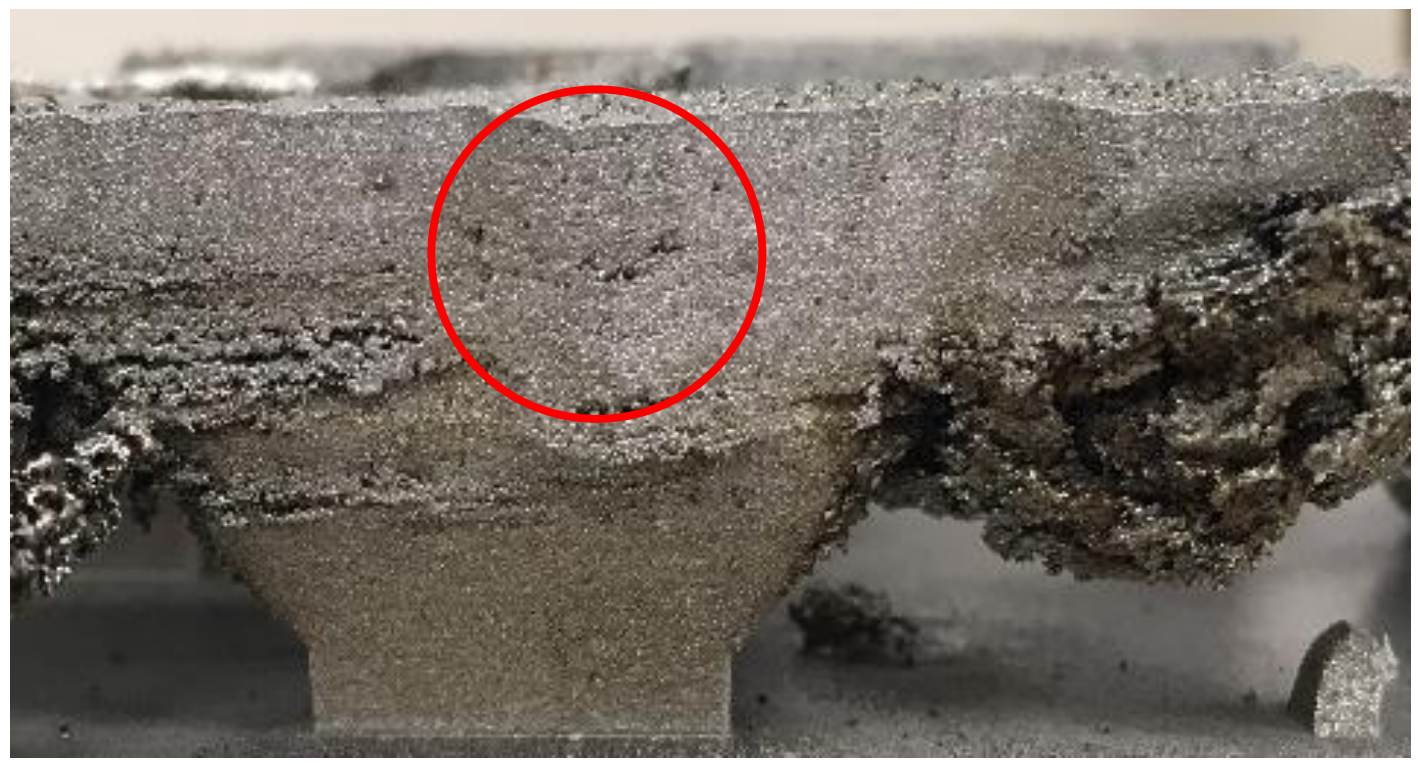

Figure 18. A photograph of a part printed past warpage demonstrates how a torn recoater blade can result in uneven powder spread. The layer boundaries in the part are no longer horizontal or even. The layers in the circle are lower than the surrounding layers.

The small cross sections of the legs are weak until they are welded together at the beginning of the larger upper section of the test coupon. Any contact of the leg's top surface with the recoater blade will cause the legs to bend. The amount of force needed to fracture the leg cross section in pure bending was estimated using simple mechanics of materials linear approximations. At the point farthest from the small cross section, a force of only $23 \mathrm{lbs}(105 \mathrm{~N})$ in the horizontal direction is required to initiate a break in the leg in a bending induced tensile failure (Appendix A). After repeated bending, the leg eventually broke away from the test coupon. Based on images taken after each recoater movement, a single recoater stroke was able to move the broken piece, $10 \times 10 \mathrm{~mm}^{2}$, up to $5 \mathrm{~mm}$ horizontally (Figure 19). 


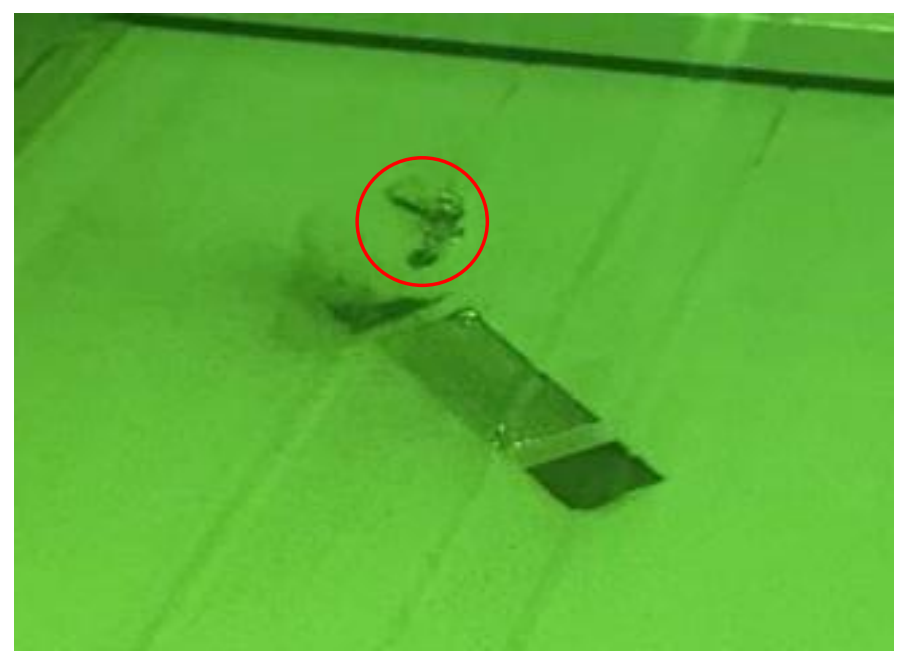

Figure 19. During the build, a piece is seen to be disconnected from the test coupon in a photo of the top powder layer. In this picture, the leg is pushed on the backwards recoat completely out of its original position.

When the leg is moved out of its original position, it leaves a void in the powder bed. The void is slowly filled with powder each time the recoater moves, causing areas behind it to not get enough powder. When the laser traces out the cross section in the void area, the melt pool is thermally insulated by the surrounding powder and remains hot for longer than other areas. This creates a large melt pool that changes from yellow to red in color (Figure 20). The larger melt pool creates uneven heating in the powder bed and can result in further warpage in other test coupons of the build. The depth of the void blocks the Argon from moving ash away from the melt pool, resulting in ash mixed into the test coupon. In addition, the part of the test coupon printed on the loose powder in the void is not supported and can continue to shift. 


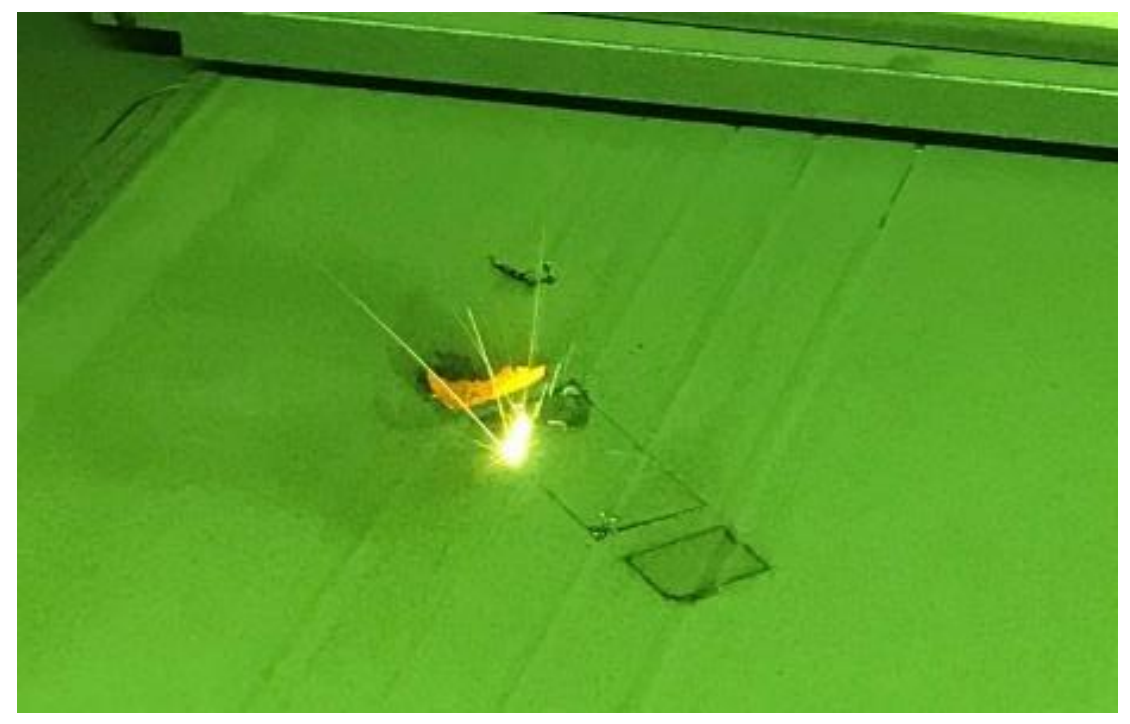

Figure 20. A photograph of the top powder layer during the build reveals the void, left from the moved test coupon piece, remains hot longer than the other test coupons of the build.

Warpage occurred when using both the stripe and chess print parameters. It was more severe with the stripe parameters. This could be due to the unevenness heat distribution in the stripe pattern that the chess pattern provides. While warpage was not a planned defect originally in the study, it proved to be more complicated and prominent than cracking.

\subsection{Cracking in Narrow Section Due to Heat}

Like warping, cracking occurs when the part shrinks as it cools. Instead of warping upwards, the part will crack at lower positions in the part. This crack relieves the internal stress in the part caused by the shrinkage. During cooling, the top portion of the part is placed in tension. It needs to shrink inward to relieve thermal stress however is held back by the lower portions rigidly attached to the build plate. This is juxtaposed with the lower section of the build that is in compression and resisting further shrinkage driven by the large top section. A crack will allow the top section to further shrink without stressing the bottom section further. 
Cracks are prominent in parts with large masses and areas with small cross sections.

Large masses retain heat for an extended time. This creates a larger heat differential between the large mass area and the build plate. Sections of the part in between these two areas are prone to cracking as the large mass shrinks while cooling. Unlike small cross sections, a large bulky cross section cannot warp as easily; therefore, it may relieve the stress by cracking.

The test coupon was designed with a large mass suspended away from the build plate by a thin cross section. As the large mass cools, it shrinks and does not warp. The small lower cross sections are broken as the top pulls inward (Figure 21). Based on simple linear estimations, the force required to initiate a crack on the outer edge of the leg is only $15 \mathrm{lbs}$ or $68 \mathrm{~N}$ at the middle of the heat reservoir (Appendix A).

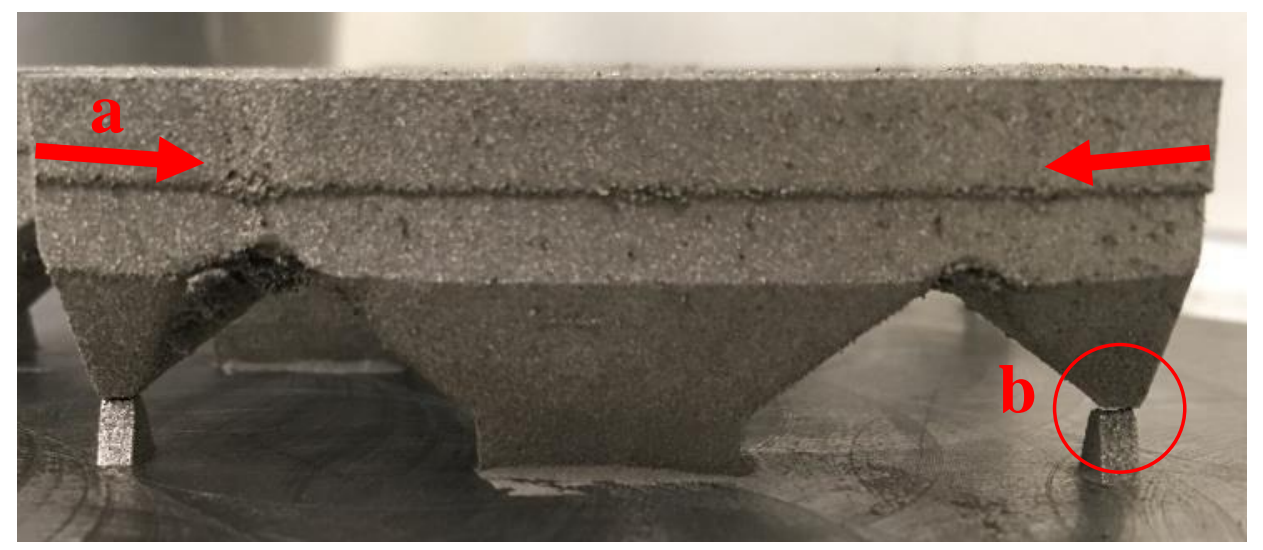

Figure 21. A photograph of the completed test coupon. The upper portion of the test coupon shrinks during cooling (a), inducing a crack in the lower weak section of the test coupon (b). The crack relieves thermally induced stress in both the upper portion and lower portion of the test coupon due to inconsistency during shrinkage.

The presence of defects in the test coupons were determined visually after the build was removed from the machine. A crack is found by the crack in the cross section, as well as a line higher in the test coupon at the layer being printed during the crack event (Figure 
22). This line is due to the test coupon shifting when it cracks. Due to lack of real time part feedback, the SLM continues to print in the pre-designated location. The result is the bottom section is not perfectly aligned with the top portion of the test coupon.

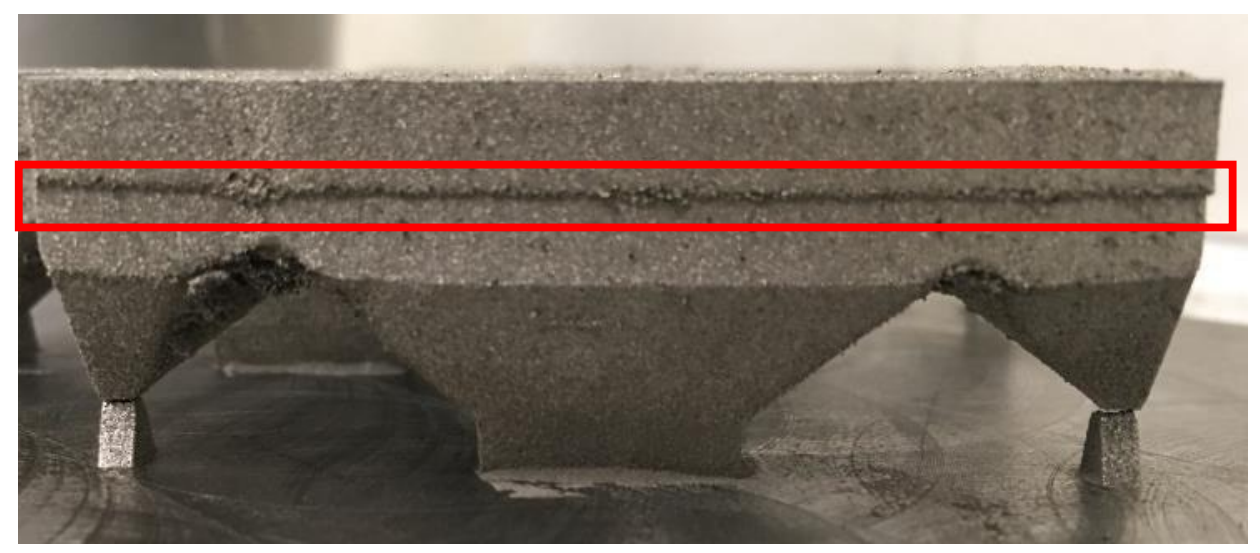

Figure 22. In the photograph of the completed test coupon, a shift is visible at the layer that the test coupon cracked. The line is due to the test coupon moving during the build due to the crack event without the printer's knowledge.

Cracking occurred in the lower weak cross sections of the test coupon. Cracking is a way for the test coupon to relieve internal stress caused by shrinking of the test coupon while it cools. Only one test coupon in the project experienced cracking. The other test coupons had severe warpage that caused the leg to be broken by the recoater blade before cracking could occur.

\section{$\underline{\text { 3.3 Material Failure Modes }}$}

To determine the failure mode of the warped and cracked cross sections, the fracture surfaces were analyzed using visual techniques. A Micro-Vu Vertex 312, an optical measurement device, was utilized to take detailed images of the cracked legs. From the Micro- $\mathrm{Vu}$ images it was found that minor elongation had occurred in the cracked test 
coupon prior to fracture (Figure 23). This indicates that the test coupon underwent plastic deformation.

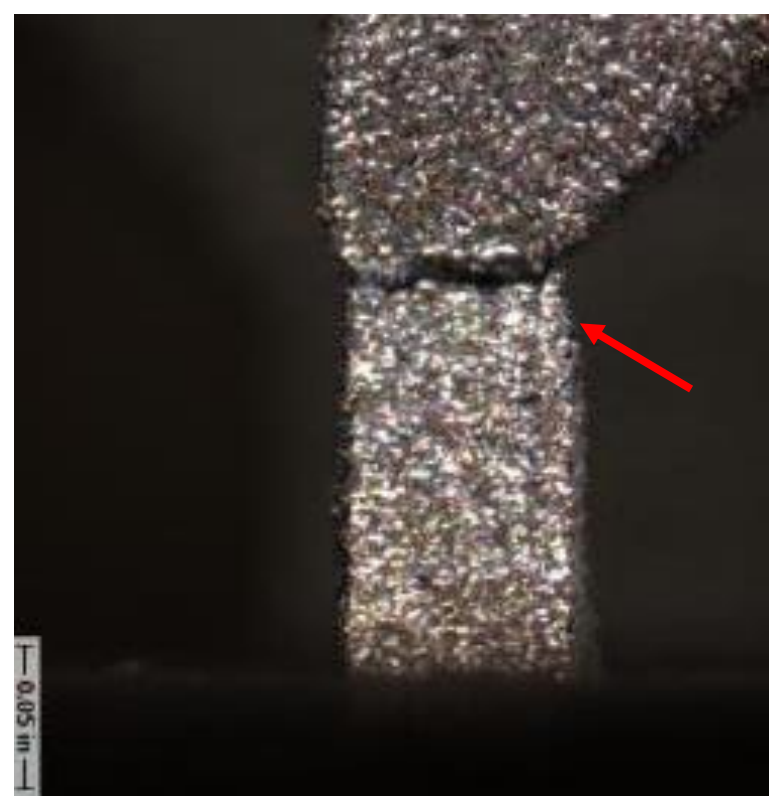

Figure 23. A Micro-vu image of a leg shows the difference in thickness between the upper and lower layers. The upper layers have undergone plastic deformation and are elongated, reducing their thickness.

Likewise, the leg broken in warpage also underwent a plastic failure before fracture. The breaking of the upper leg section off due to the recoater movement is evident in the photos of the remaining leg stub. The leg is bent sideways and elongated, indicating that the leg was placed in plastic deformation before breaking (Figure 24). 


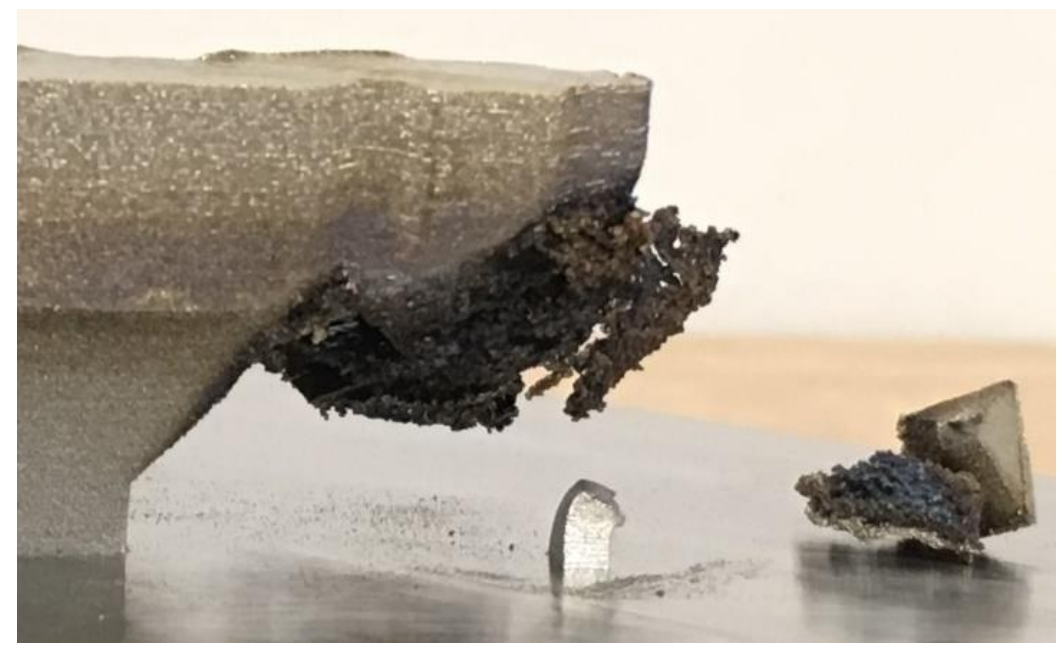

Figure 24. A photograph of the remaining leg stub from the severe warpage cases suggests that it is bent slowly over time, allowing it to undergo plastic deformation.

The scanning electron microscope (SEM) results indicate that both the warpage and the crack legs fractured due to a tensile normal stress failure. The smooth surface on one side of the crack's cross section signifies a fast brittle failure (Figure 25). The voids and elongation on the other half of the surface are due to a ductile failure. The crack started with the brittle failure first, and then changed into a ductile failure in the second half of the cross section. These results mean that the cause of failure could be either bending or a pure tensile pull. Due to the position of the legs, however, that are slightly bent outward, it can be assumed that the crack failure is a combination of bending and tensile loads. 


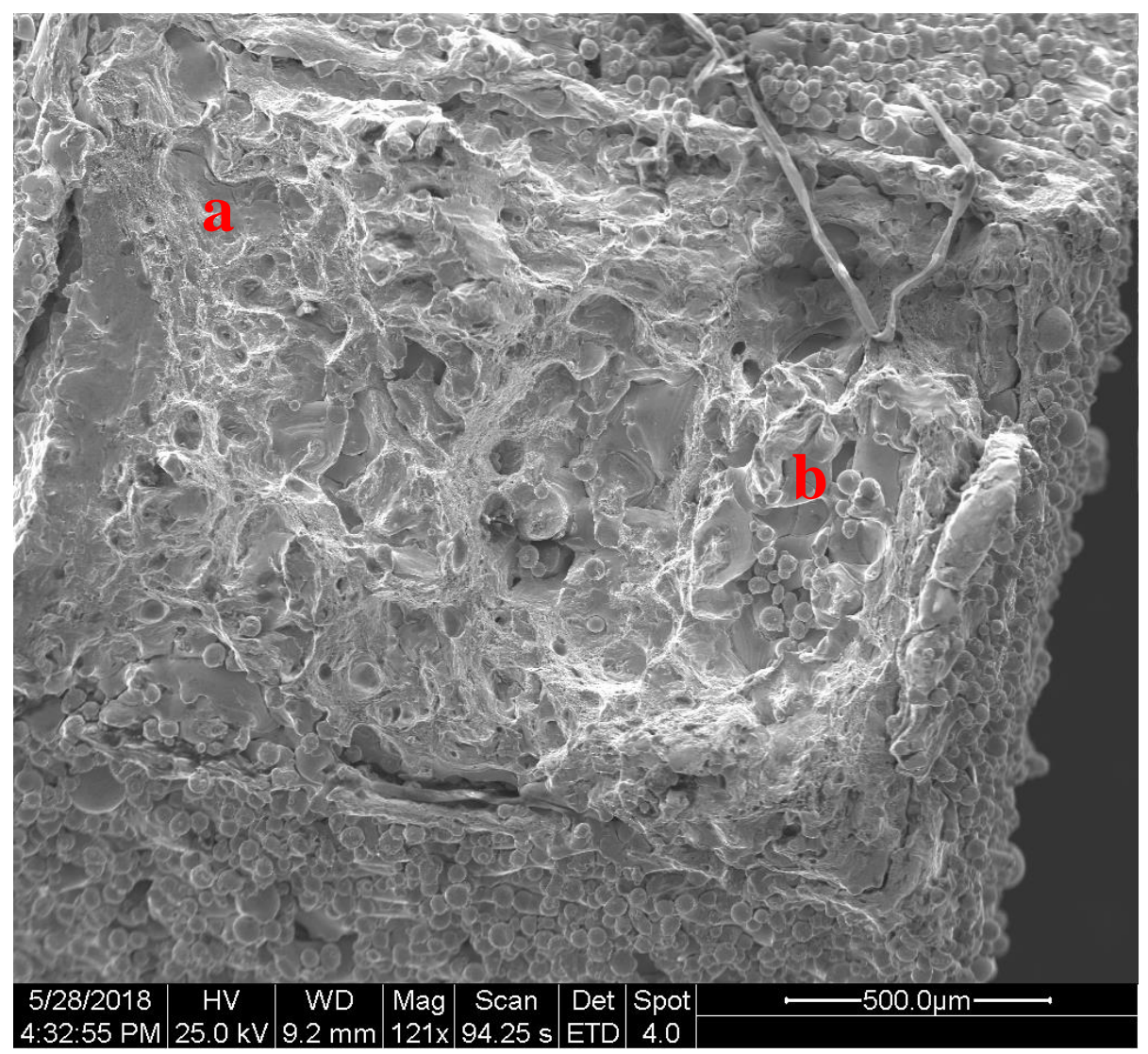

Figure 25. The SEM photos of the crack surface shows that the failure was a brittle (a) to ductile (b) tensile failure. (Yamanaka)

SEM photos of the heat affected zone indicate that the powder surrounding the edges and ridges of the test coupons were heated repeatedly. This caused powder particles to conglomerate (Figure 26). Particles like these are sieved out in between builds because they can cause issues in spreading the powder in a $30 \mu \mathrm{m}$ layer. The continuous cycle of heating and cooling can also change the properties of the powder, and over time change the properties of the final part. The many edges and corners of the test coupon led to repeated laser exposure in surrounding areas and resulted in a burned appearance in the test coupon. 


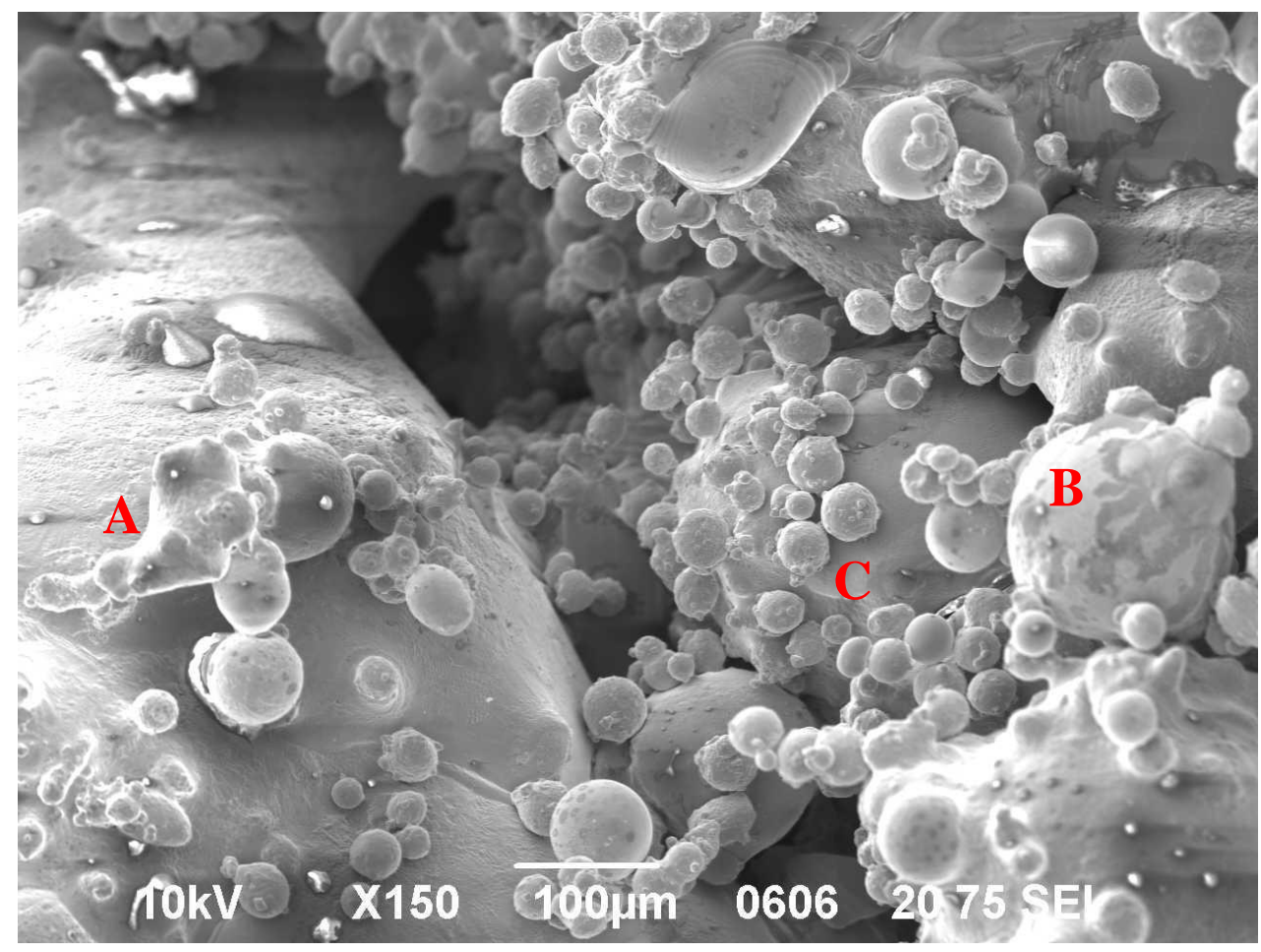

Figure 26. A SEM image shows the irregular conglomerates of particles (A) are created when they are heated enough to partially fuse together. Heat affected particles $(B)$ occur when the particle is melted multiple times. Normal Particles $(C)$ are separate and free flowing.

\section{$\underline{3.4 \text { Summary of Findings }}$}

The first phase of this project to induce a predictable failure in the test coupon was not fully successful. While the two defects of warpage and cracking were produced, the additional side effects and unpredictability of the defects were not ideal. Severe warpage caused the leg to be torn off the test coupon in nine out of the ten builds, preventing any cracks from occurring. Overall, the five test coupons printed using the chess parameters had less warpage than the five printed with the stripe parameters setting. Only one out of ten builds were able to print past warpage and experience a crack. 


\section{Chapter 4: Detecting Part Failure}

In this chapter, analysis of accelerometer, image, and audio data collected during each build is discussed. The analysis occurred after the test coupon was removed from the printer and visually inspected for defects, specifically warpage and cracking. The sensor data was compared to the machine script and the post-print visual inspection to determine when the defect had occurred. To supplement the frequency characterization of signal data, three different frequency techniques are discussed. A FEA model was also used to verify findings. The future goal of this research is to implement the data analysis in situ, enabling part failures to be detected in real time.

\section{$\underline{4.1 \text { Accelerometer Analysis }}$}

Accelerometer data was the primary method of defect detection in this study. The accelerometer data was collected at $12500 \mathrm{~Hz}$ and stored on an external hard drive. Time and frequency domain analysis were used to characterize distinct sections of the accelerometer profile. As discussed below, the events in the accelerometer data were able to be identified through comparison to the SLM Solutions machine script.

\subsubsection{Experimental Set $U p$}

The two accelerometers used in this experiment were a Kistler 8766A500AH 500g PiezoStar@ triaxial accelerometer and 8278A500sp5m uniaxial accelerometer. A previous mechanical engineering senior project, sponsored by Lawrence Livermore National Laboratory, purchased and installed the two accelerometers on the SLM Solutions machine. The triaxial accelerometer was mounted to an accelerometer mount using 5 - 40 threads tapped into the accelerometer (Coria, Whipple and Grant). A small 
break in the uniaxial accelerometer cable rendered it useless partway through data collection. The uniaxial accelerometer and the triaxial accelerometer base were attached to the bottom of the build platform using silicone adhesive (Figure 27). The location was chosen because the sensors could not be placed in closer proximity to the part without causing permanent alterations to the SLM Solutions machine. Loctite silicone sealant was used because the attachment location can reach up to $200^{\circ} \mathrm{C}$ during a build.

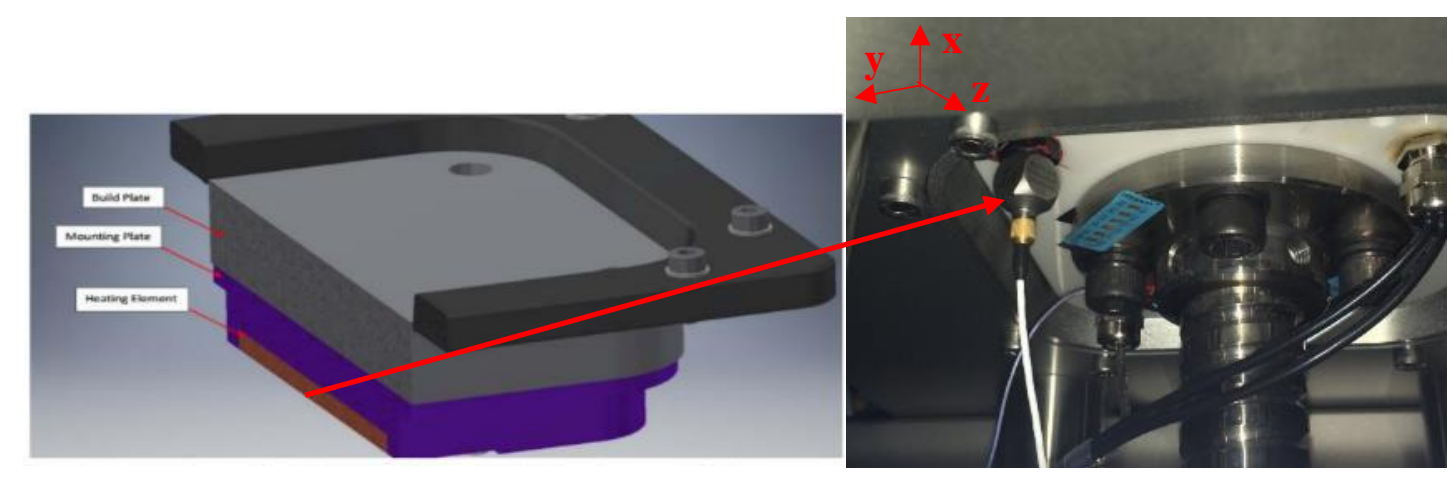

Figure 27. The accelerometer's location on the printer is compared with its location in the CAD model. The accelerometers were mounted using silicone adhesive. The orientation of the triaxial accelerometer has the $x$ axis in the upwards direction. The $y$ axis is skewed 45 degrees clockwise about the x axis. (SLM Solutions Group)

The orientation of the triaxial accelerometer has the y axis approximately 45 degrees to the recoater blade with the $\mathrm{x}$ axis in the upward vertical direction (Figure above). The choice of orientation is not clear from the previous report. For this study, only data in the $\mathrm{x}$ direction was considered. A directional study found that when the build plate was tapped in the build chamber's $\mathrm{x}, \mathrm{y}$, and $\mathrm{z}$ directions for a modal hammer test, the accelerometers all registered approximately the same response (Figure 28). Likewise, data of a potential crack had similar magnitudes in all three directions with the vertical direction being the most sensitive. This sensitivity could be due to less rigidity in the build platform structure in the vertical direction. In the horizontal directions the build 
platform is restricted by the build envelope, but in the vertical direction, the build platform can move using a lead screw.

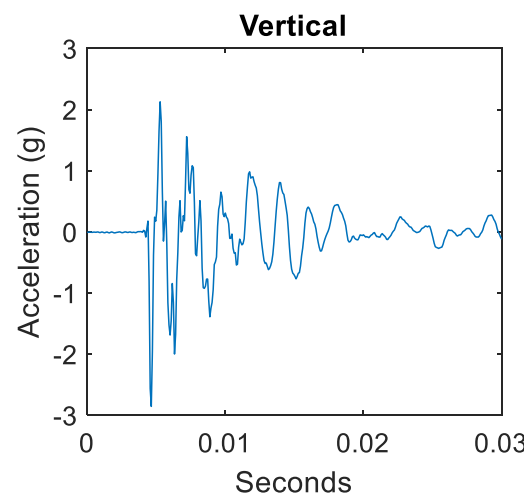

$a$

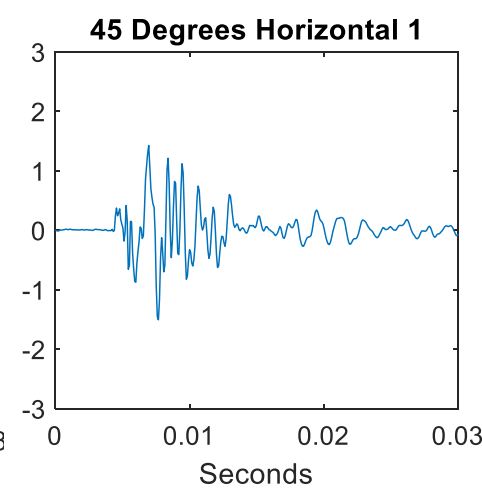

$B$

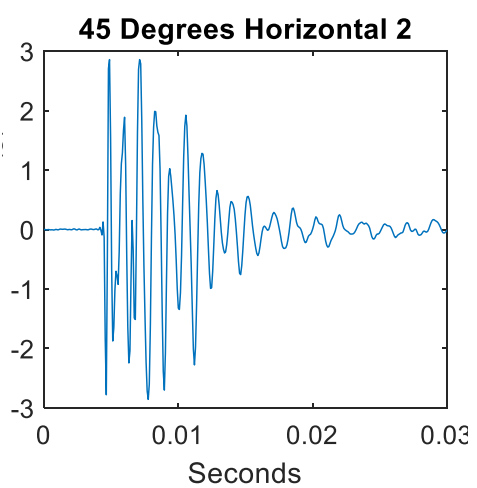

$c$

Figure 28. Three acceleration versus time plots recording the same event in time are compared. The triaxial accelerometer measured a tap of the modal hammer in all three directions with similar magnitude.

Data was collected from the three accelerometer channels at $12500 \mathrm{~Hz}$. The DAQ could provide data up to $62500 \mathrm{~Hz}$, however, a FIFO data transfer error occurred when a sample rate higher than $12500 \mathrm{~Hz}$ was used. A FIFO error, or First In First Out, indicates that data was entering the system at a faster rate than the computer could process it. Over time, the difference in speeds causes there to be a backup of data entering the system and any new data sent to the system is rejected (National Instruments). For example, a computer can process data at $62500 \mathrm{~Hz}$ for only 30 minutes but at $25000 \mathrm{~Hz}$ for 2 hours. The 11 hour duration of the test coupon's build meant that the data could only be collected at $12500 \mathrm{~Hz}$ using the current computer. The risk of using a slower data collection rate in signal analysis is the possibility of aliasing. Aliasing occurs when data is taken at a lower frequency than the input signal frequency (Wickert). For example, if a 
computer records only two data points per period in a sine wave, the recorded signal may appear as a different sine wave than the actual input sine wave (Figure 29).
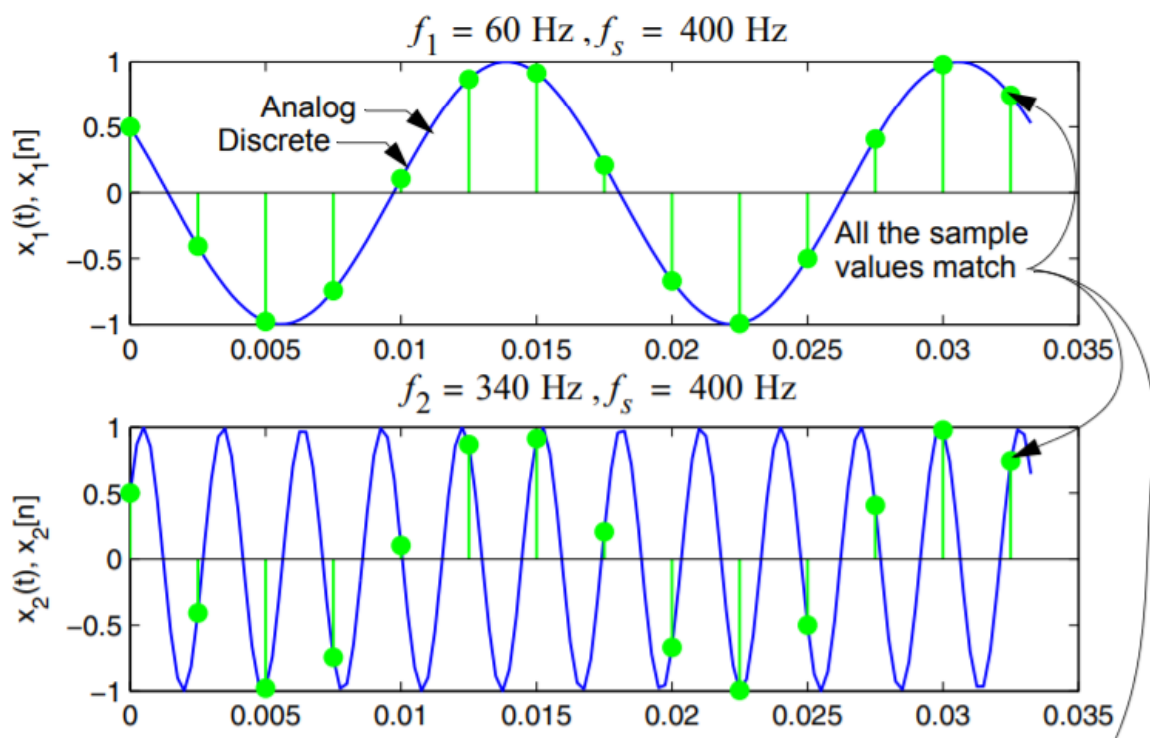

$f_{3}=460 \mathrm{~Hz}, f_{s}=400 \mathrm{~Hz}$

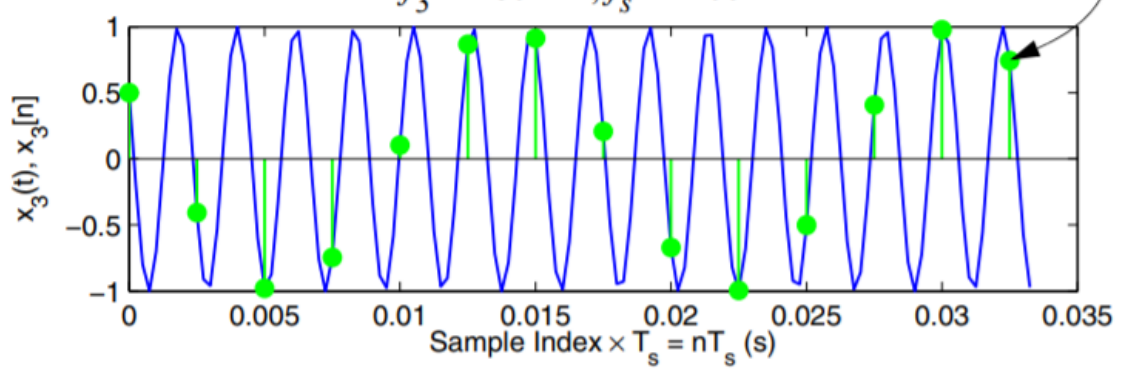

Figure 29. Two example signals, green and blue, are compared in the time domain. Aliasing is a problem that can occur in signal analysis when a sample rate is too low to obtain the actual shape of the input data. In the above example, the green samples do not adequately show the true shape of the blue input data. (Wickert)

Another concern in data collection was the presence of unrelated background noise, such as people entering the SLM Solutions room, to appear in the data. A test was conducted in which potential vibrations were created by shutting closed the SLM cabinet doors, stomping the floor, and tapping the side of the machine. While some of the noises produced were evident when reviewing the data, their magnitude was well below the 
magnitude of normal machine operating vibrations of $0.05 \mathrm{~g}$ (Figure 30). Given the results of this test, it was concluded that unrelated background noise was not a concern in the data set.

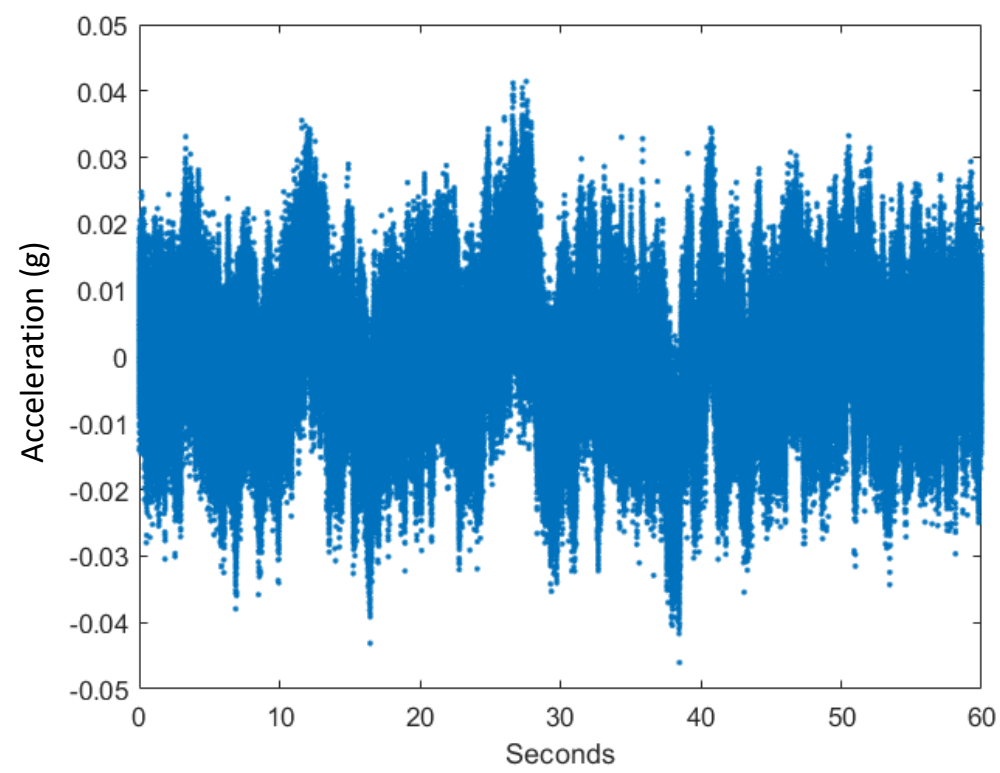

Figure 30. An acceleration versus time plot of a noise test demonstrated that movements produced by unrelated noises would not produce a signal above the amplitude of the normal operating machine noise which is $0.05 \mathrm{~g}$.

\subsubsection{Frequency Analysis Methods Overview}

To characterize the three distinct phases of the accelerometer profile as well as determine whether an acceleration spike is due to a crack, three different methods of frequency domain analysis were used: Fourier Transform, Wavelet Decomposition, and Shock Response Spectrum. An overview of each of these methods are discussed in this section.

\subsubsection{Fourier Transformation}

Fourier Transform represents an input function into a series of sine and cosine functions. In the process, the function is transformed into the frequency domain before it is moved 
back to the time domain. For signal analysis, the Fourier Transform creates a method to determine the most prominent frequencies in the input. Continuous Fourier Transform uses a function $f(t)$ and gives its frequency representation $F(n)$ :

$$
F(n)=\int_{-\infty}^{\infty} f(t) e^{-2 \pi i f t} d t
$$

In discrete signal data, as received from the accelerometer, Discrete Fourier Transform is used. The discrete method uses the same equation as the Continuous Fourier Transform except for a summation sign instead of an integral (Roberts):

$$
F[n]=\sum_{k=0}^{N-1} f[k] e^{-j\left(\frac{2 \pi}{N}\right) n k} \quad n \in[0, N-1]
$$

A Fourier Transform of the data is displayed on two different spectrums: magnitude and phase. The magnitude at each frequency is seen on a magnitude versus frequency plot (Figure 31). Signal frequencies with more occurrences have a higher magnitude on the plot. From the plot, the signal frequencies can be characterized more easily than in the time domain. In the time domain, the multiple input frequencies are composed together to create the signal. In the frequency domain, however, these frequencies are separated and displayed individually. 


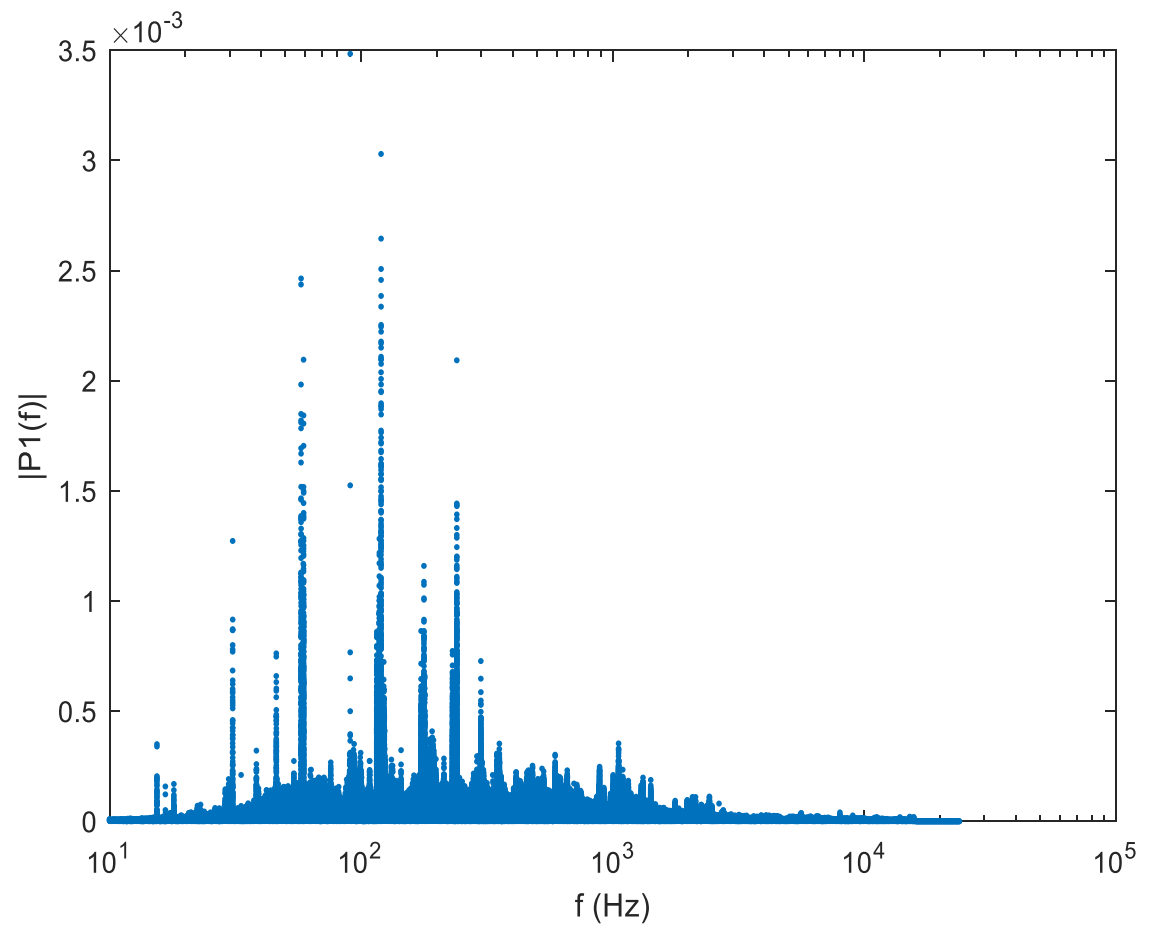

Figure 31. The Fourier Transform of a signal results in a magnitude vs. frequency plot. In addition to the frequency spectrum, the Fourier Transform also creates a phase spectrum (Figure 32). The phase spectrum dictates each frequency's time delay. The relative phases of the frequencies will determine whether the frequencies' peaks will add or subtract. The addition and subtraction of the different frequencies creates the unique pattern of the signal. Two signals may have the same magnitude spectrum but will have different shapes based on the phase spectrum. The combination of phase and magnitude spectrums make Fourier Transform a useful in analyzing data in the frequency domain. 


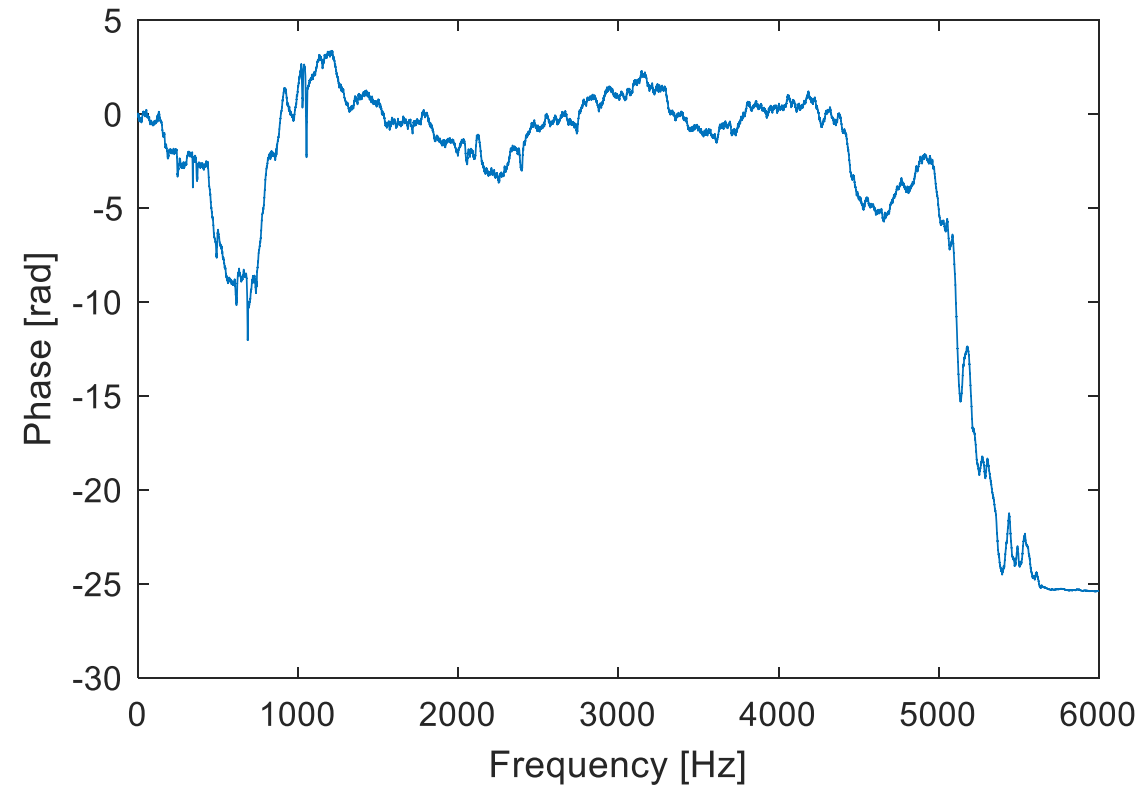

Figure 32. A phase spectrum displays the time delay of each frequency. The unique combination of phase and magnitude of different frequencies creates an individual signal.

\subsubsection{Wavelet Decomposition}

Like Fourier Transform, Wavelet Decomposition is a function transformation from the time domain to the frequency domain. The equation for the wavelet transform is (Sadowsky):

$$
W(a, b)=\sqrt{a} \int_{-\infty}^{\infty} S(\omega) \Psi *(a \omega) e^{i \omega b} d \omega
$$

A wavelet is a collection of sine and cosine functions that create a peak that is present in one instance of time. This differs from a Fourier transformation when the waves are repeating throughout time. Its transient behavior is the reason wavelet analysis is preferred to Fourier analysis when observing sudden or non-repeating events in time. 
Wavelet decomposition matches a pre-formed wavelet to the signal by scaling and shifting it (Devleker).

The wavelet transformation used in this paper is the Morse Analytic wavelet. The Morse wavelet is used in continuous wavelet transformation because it is versatile due to its exactly analytic nature (MathWorks). A Morse Wavelet is defined by the following equation:

$$
\Psi_{P, \gamma}(\omega)=U(\omega) a_{P, \gamma} \omega^{\frac{P^{2}}{\gamma}} e^{-\omega^{\gamma}}
$$

It is the default family of wavelets used in continuous wavelet transformation function, cwt.m, available in Matlab. The transient nature of the wavelet comes from the unit step function, $U(\omega) \cdot a_{P, \gamma}$ normalizes the transformation. The symmetry parameter, $\gamma$, and time-bandwidth, $P$, are used to stretch and shift the wavelet as needed to fit the signal (Figure 33). Altering $\gamma$ from perfect symmetry at $\gamma=3$ will change the time decay of the signal in the frequency time domain. Decreasing the time-bandwidth will shrink the width of the wavelet and lower the frequency. 

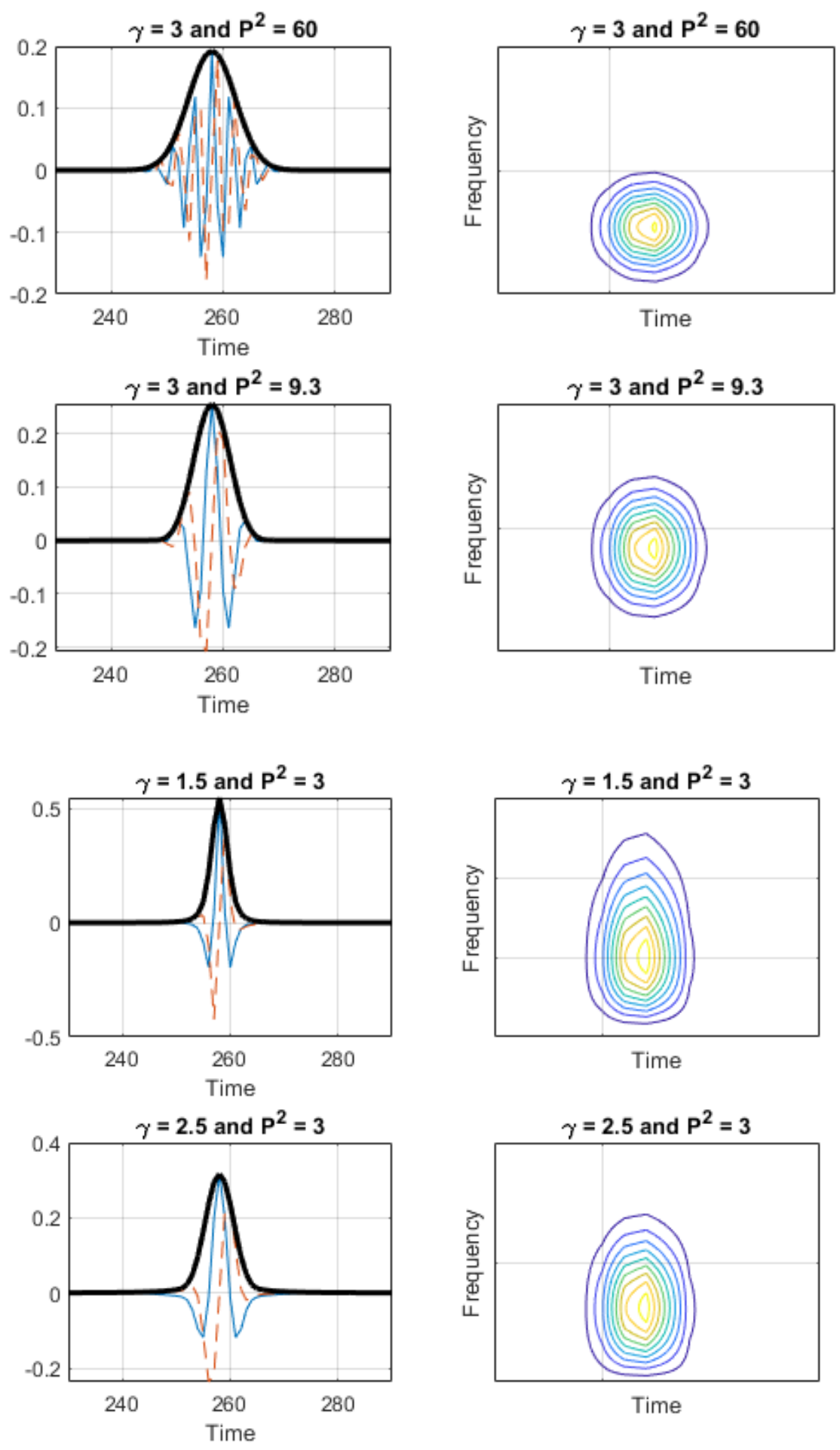

Figure 33. A time domain plot of the signal with a Morse Wavelet superimposed (left) is then transformed into a frequency versus time domain plot (right) using Wavelet Decomposition. The Morse Wavelet can match a signal response by varying the gamma and $P$ parameters. (MathWorks) 
The Matlab function cwt.m, was used to transform data from into the frequency domain. The function produces a frequency versus time colormap of the accelerometer data using the same equation described above (Figure 34). Frequencies with higher magnitudes will be yellow and frequencies with lower magnitude will be blue. The time-frequency relationship of the Wavelet Transformation is one of its unique features that make it preferred for transient responses.
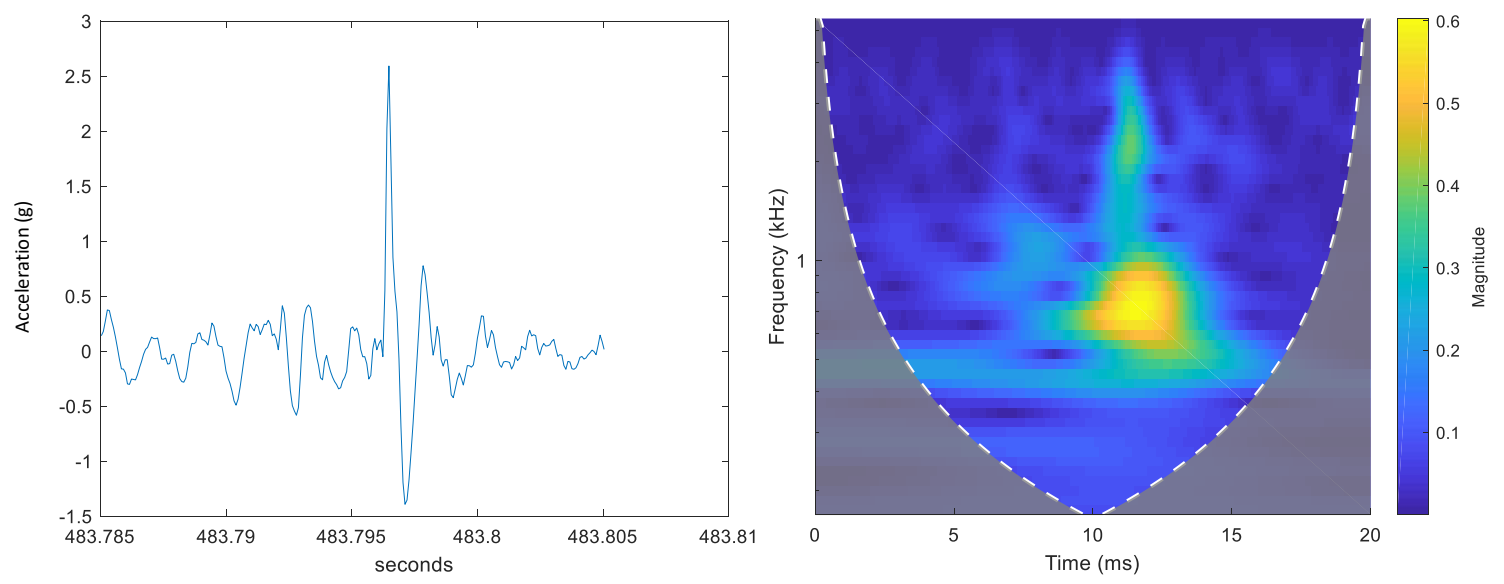

Figure 34. The time domain plot of a spike is transformed into the frequency vs. time plot by the function cwt.m. The frequency axis of the Wavelet Plot is a log scale.

\subsubsection{Shock Response Spectrum}

Shock Response Spectrums (SRS) are used for impact events such as earthquakes. The goal of the SRS is to split a shock into its natural frequency components. SRS uses a simple mass spring damper model to do this (Figure 35). 


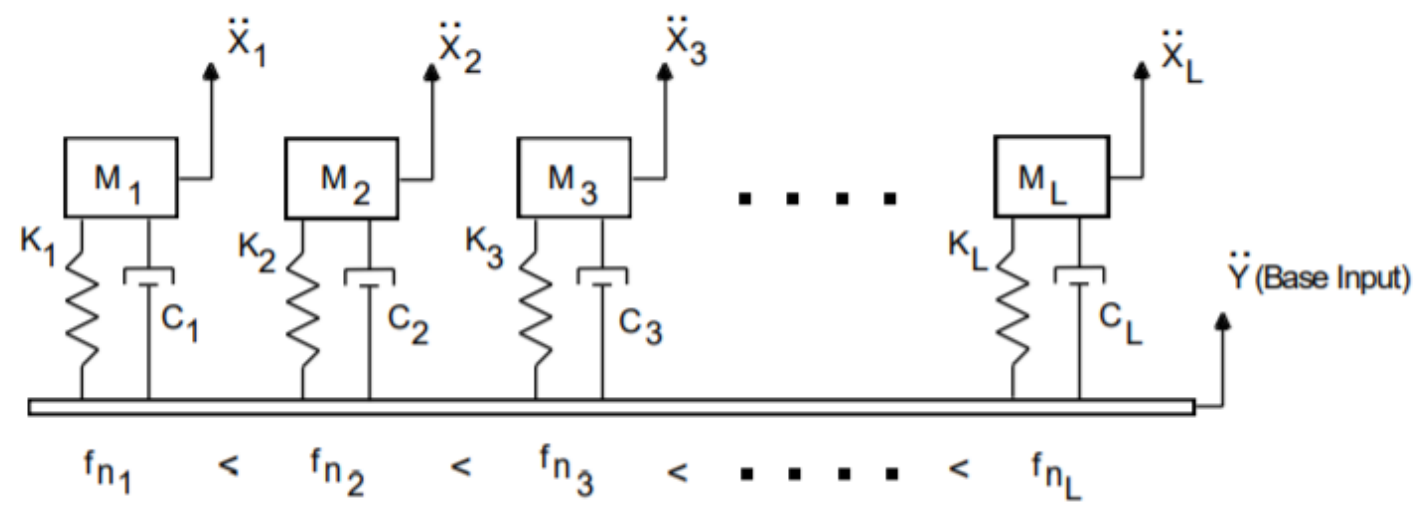

Figure 35. The SRS model uses multiple mass-spring dampers to simulate the excitation of different natural frequencies (Irvine).

A benefit of SRS is low computation power compared to transforms because the SRS uses a simpler equation to complete the decomposition. SRS solves the following equation of motion (Irvine):

$$
\ddot{z}+2 \xi \omega_{n} \dot{z}+\omega_{n}^{2} z=-\ddot{Y}(t)
$$

The accelerometer data is the input $(\ddot{Y})$ into a bar. Attached to the bar are a variety of mass-spring systems. Each mass-spring has its own natural frequency $\left(\omega_{n}\right)$. When the bar is moved according to the accelerometer signal, the masses (M) with matching natural frequencies as the input will be more excited. Each mass includes a spring $(\mathrm{K})$ and a damper (C). The damping is assumed to be $5 \%$. The acceleration of the masses is symbolized by $\ddot{X}$. 
A convolution integral is used to solve the equation of motion (Irvine):

$$
\begin{aligned}
\ddot{X}_{i}=2 \exp (- & \left.\xi \omega_{n} \Delta t\right) \cos \left(\omega_{d} \Delta t\right) \ddot{X}_{i-1}-\exp \left(-2 \xi \omega_{n} \Delta t\right) \ddot{X}_{i-2} \\
& +2 \xi \omega_{n} \Delta t \ddot{Y}_{i} \\
& +\omega_{n} \Delta t \exp \left(-\xi \omega_{n} \Delta t\right)\left(\left(\frac{\omega_{n}}{\omega_{d}}\left(1-2 \xi^{2}\right)\right) \sin \left(\omega_{d} \Delta t\right) \quad \text { Eq. } 6\right. \\
& \left.-2 \xi \cos \left(\omega_{d} \Delta t\right)\right) \ddot{Y}_{i-1}
\end{aligned}
$$

In the Matlab function srs.m downloaded from Tom Irvine on the Mathworks File Exchange, the natural frequencies are chosen to be at 1/6 octaves (Irvine). This means that frequency two is $2^{\wedge}(1 / 6)$ times frequency one. The program limits the highest frequency to be one tenth of the signal's sample rate. Frequencies higher than one tenth of the sample rate are more likely to encounter aliasing issues in analysis. The highest frequency the signal was compared against was limited to $1250 \mathrm{~Hz}$ based off one tenth of the sample rate of $12500 \mathrm{~Hz}$. Unfortunately, the natural frequencies indicated by the wavelet decomposition are higher: $1400 \mathrm{~Hz}, 2200 \mathrm{~Hz}$, and $3800 \mathrm{~Hz}$. In addition to concerns of aliasing in the analysis, the SRS transformation lacks the phase spectrum provided by the Fourier Transform. This missing information means that different signals can produce the same SRS plot. The lack of identification between different signals makes SRS is not suitable for the purposes of this project, even if the sample rate was increased. 


\subsubsection{Print Profile Overview}

The data sets for each test coupon had similar patterns of acceleration. In some areas, the acceleration was much greater than in others. These different regions collectively create an accelerometer print profile. In general, the print profile will vary part to part based on the location where defects occur. The builds in this project used all the same test coupons, however, so the accelerometer profiles only had slight variation (Figure 36). For this test coupon's accelerometer profile, there are three distinct regions: beginning recoater, warpage and later recoater (Figure 37).

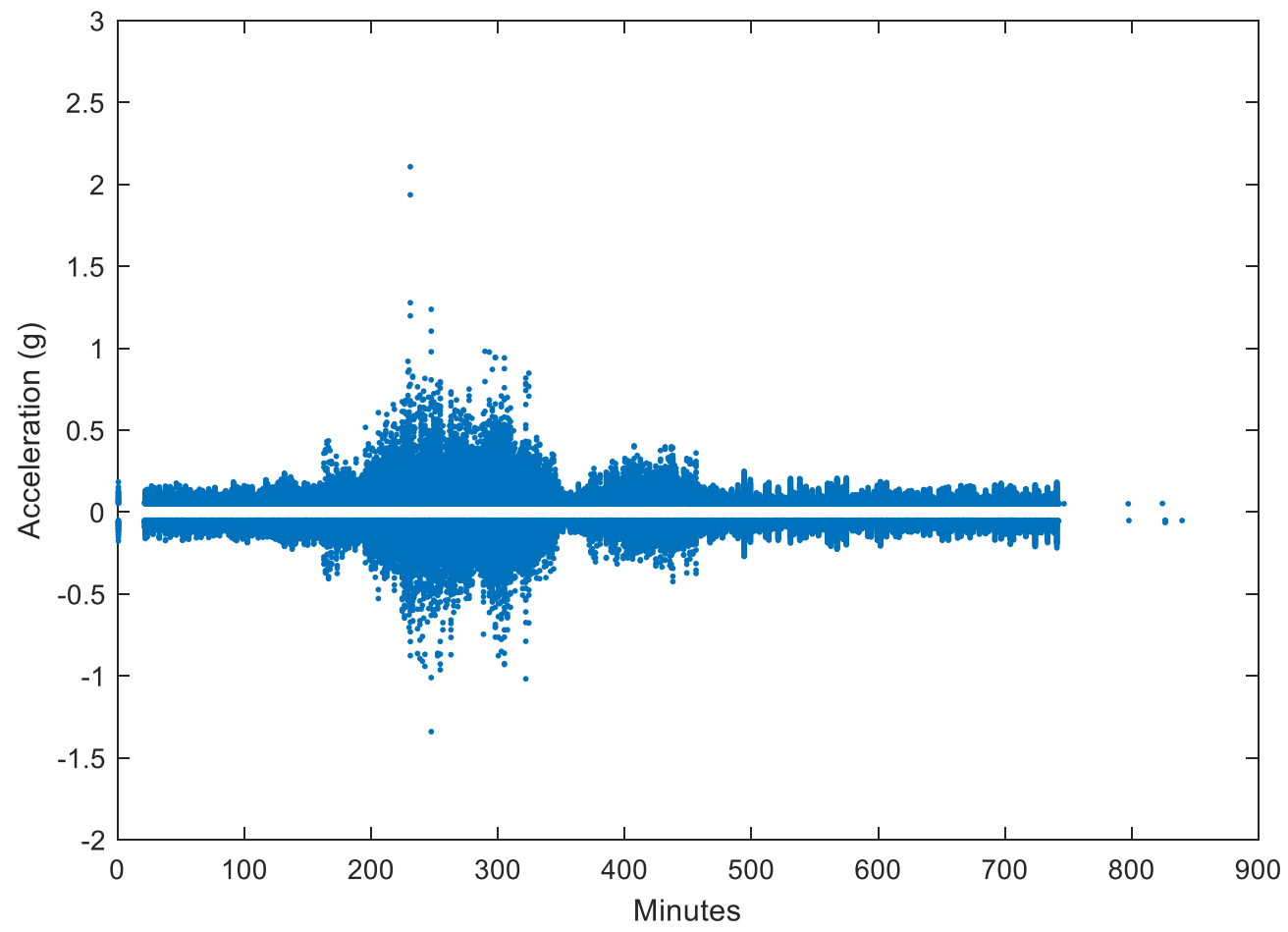

Figure 36. Plot of acceleration versus time with low amplitude filter applied for data collected during a build with severer warpage. 


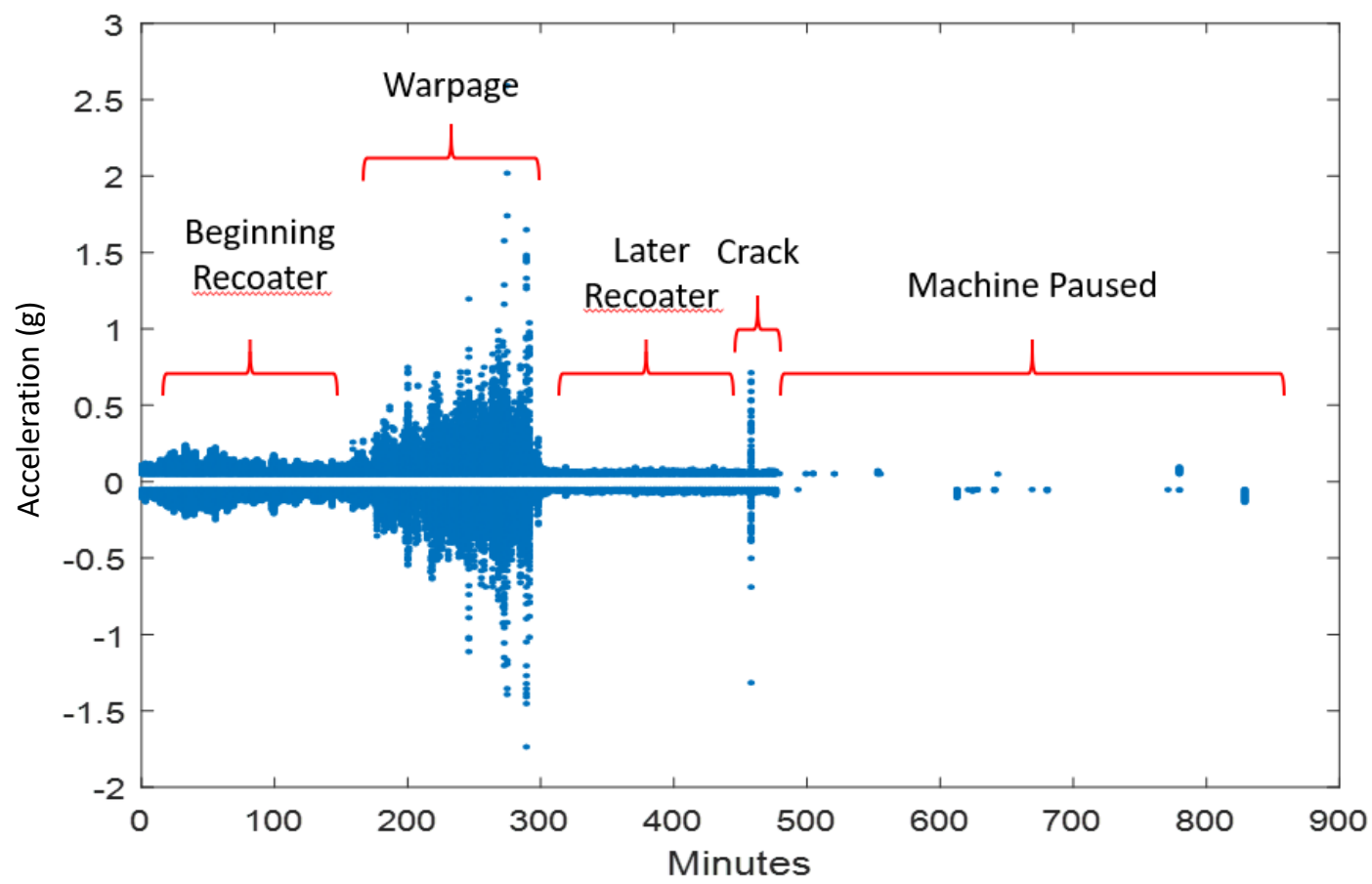

Figure 37. Plot of acceleration versus time with low amplitude filter applied for data collected during the cracked build. The build was paused just before 500 minutes and then resumed later. The remaining resumed build data is not shown. The accelerometer profile for the first part of the build has five parts based off the amplitude of the raw accelerometer data: beginning recoater, warpage, later recoater, crack, and machine paused.

The beginning recoater region is characterized by visible recoater spikes. The timing of these spikes aligns with the recoater movement times on the machine script. The recoating spikes are up to $0.2 \mathrm{~g}$ in amplitude (Figure 38 ). In the beginning of the build, the recoater blade is rubbing across the plate or is very close to the plate. The lack of powder on the plate creates a minimal vibration cushion between the recoater and the build plate. The proximity of the recoater results in larger acceleration during recoater movement than when the recoater blade is further away. 


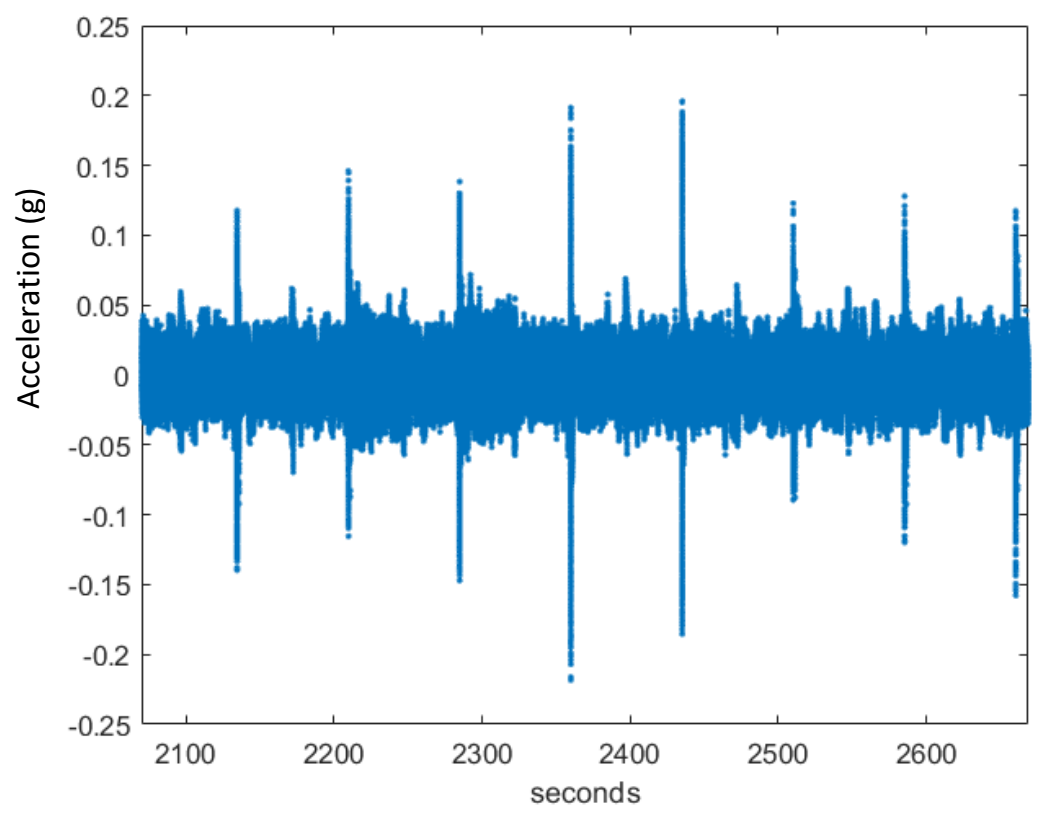

Figure 38. A close view of the beginning recoater region shows that the recoater moves every 30 seconds. This matches the frequency of the recoater commands on the machine script. The recoater spikes reach up to $0.2 \mathrm{~g}$.

Each recoater spike begins with a gradual acceleration before the peak. The peak is only

$0.1 \mathrm{~g}$ high (Figure 39). After the peak the recoater takes approximately one second to move across the plate. The recoater makes less noise as it crosses the build chamber floor than the raised build plate as seen by the lower magnitude at the beginning and end of its travel. The recoater movement ends when it hits into the stopper at the end of its travel. This recoater profile is only seen at the beginning of the build when the recoater blade is touching the build plate. 


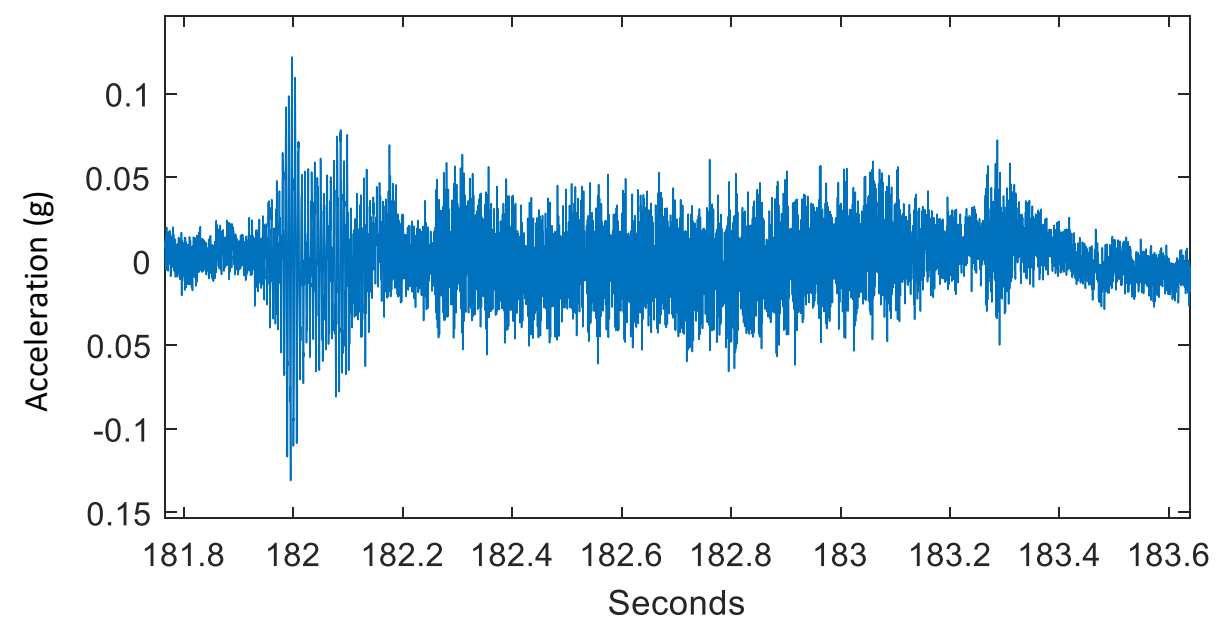

Figure 39. A closeup plot of a single recoater movement in the beginning of the print contains an initial acceleration, movement, and then an abrupt halt as it hits two stoppers.

The second region of the test coupon build shows high acceleration due to warpage.

Unlike the beginning recoater section, the warpage section is not part of a normal build's profile. This section of the accelerometer profile is present because the test coupon had a defect during this time. As discussed in Chapter 3, the warpage in the test coupon occurs when the square leg sections cool, causing them to curve upward. The recoater blade then catches on these high sections and rips. The contact between the recoater blade and the warped sections results in a large acceleration. During this section of the build, the accelerometer profile exhibits large recoater spikes magnitudes, up to 2 g (Figure 40). These spikes are ten times larger than in the beginning recoater section. The acceleration when the laser is running, or the test coupon is cooling (if the set minimum scanning time is greater than the laser time), is the same amplitude as in the beginning recoater region. 


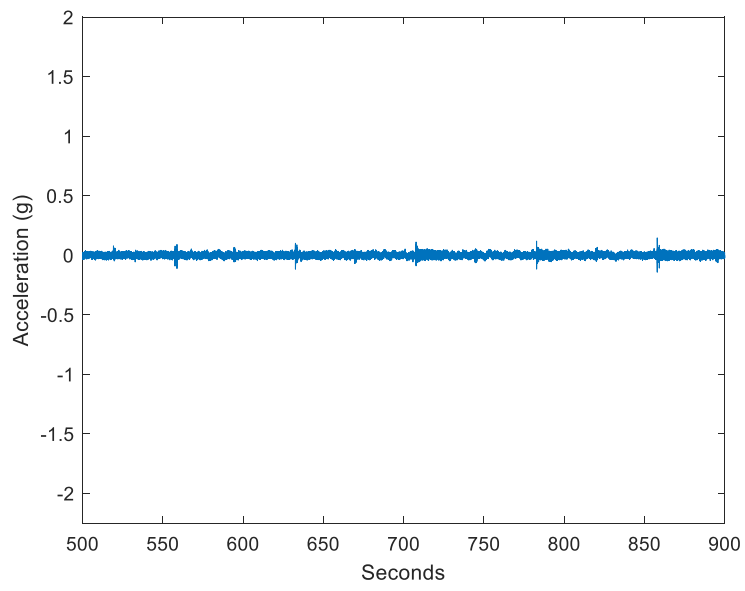

a

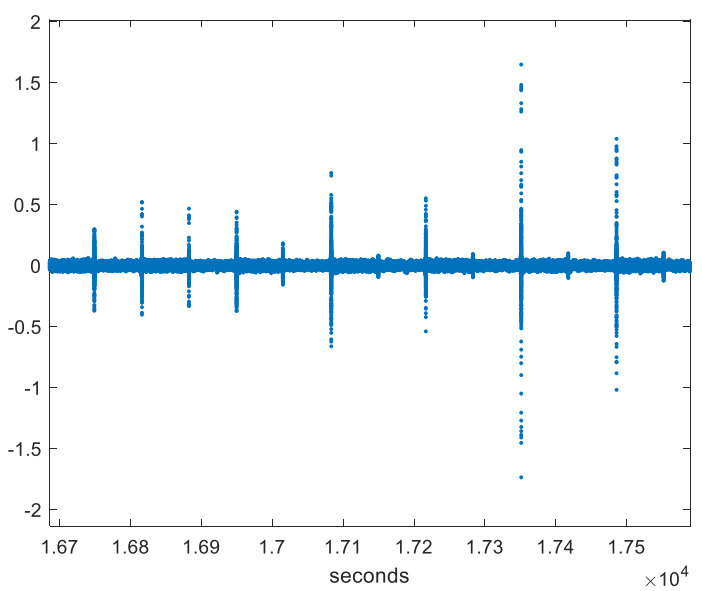

B

Figure 40. A closeup of the warpage section (b) of the print demonstrates the higher acceleration during the recoater passes than in the beginning recoater section $(a)$.

After the warpage is printed past, the acceleration reduces. This is the later recoater region. The test coupon profile is characteristic of the later recoater section when the large upper mass is printed. The later recoater profile is created when there is minimal warpage and the build plate is well covered with powder. The powder dampens the effect of the recoater, so no spikes are visible (Figure 41). Since the recoater spikes are not present, when the data is viewed closer, one can observe alternating high and low sections. The high and low sections align with the movement of the recoater. During the latter part of the build, the laser lasts 45 seconds instead of 10 seconds to complete a layer due to the increased cross section area. Since the minimum scan time is set to 30 seconds, the recoater moves to the other side of the build plate immediately once the laser is done. When the recoater is on one side of the build chamber versus the other, vibrations from 
the laser and other machine components are better damped. The alternating position of the recoater creates the high and low pattern seen in this section.

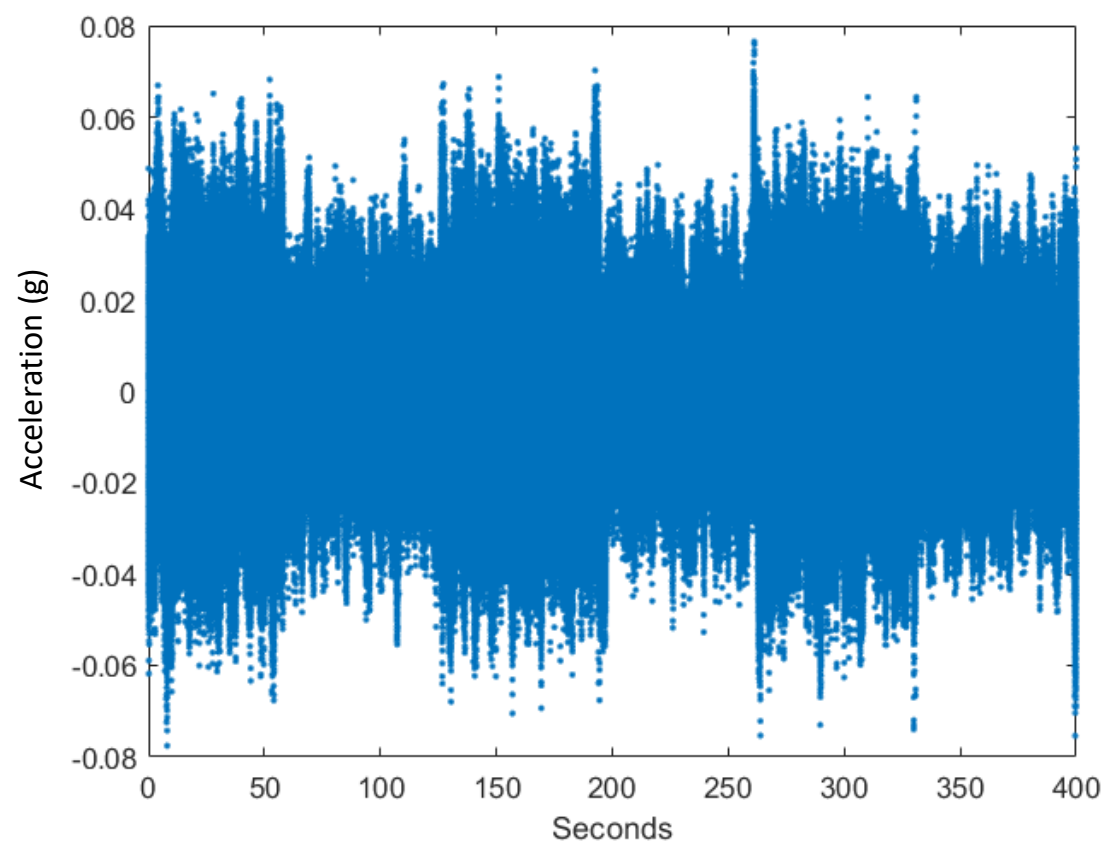

Figure 41. As seen in the closeup of the later recoater section of the build, recoater spikes do not appear in the accelerometer profile because of the large amount of powder on the build plate. Instead, the altering high and low sections are due to other machine noise being damped differently based on the recoater position.

\subsubsection{Background Machine Noise Analysis}

The main purpose of the accelerometer data is to determine when defects occur. Analysis of the machine background noise supplements this goal because it gives a comparison of the defect regions to normal machine operation noises. The difference between the SLM Solutions machine actively printing and the SLM Solutions machine paused was investigated using data from a build that had paused partway through. The build's latter recoater section served as the active machine data sample. This section was chosen 
because the pause occurred during this portion of the build. A decrease in acceleration magnitude defines the moment that the pause occurred (Figure 42).

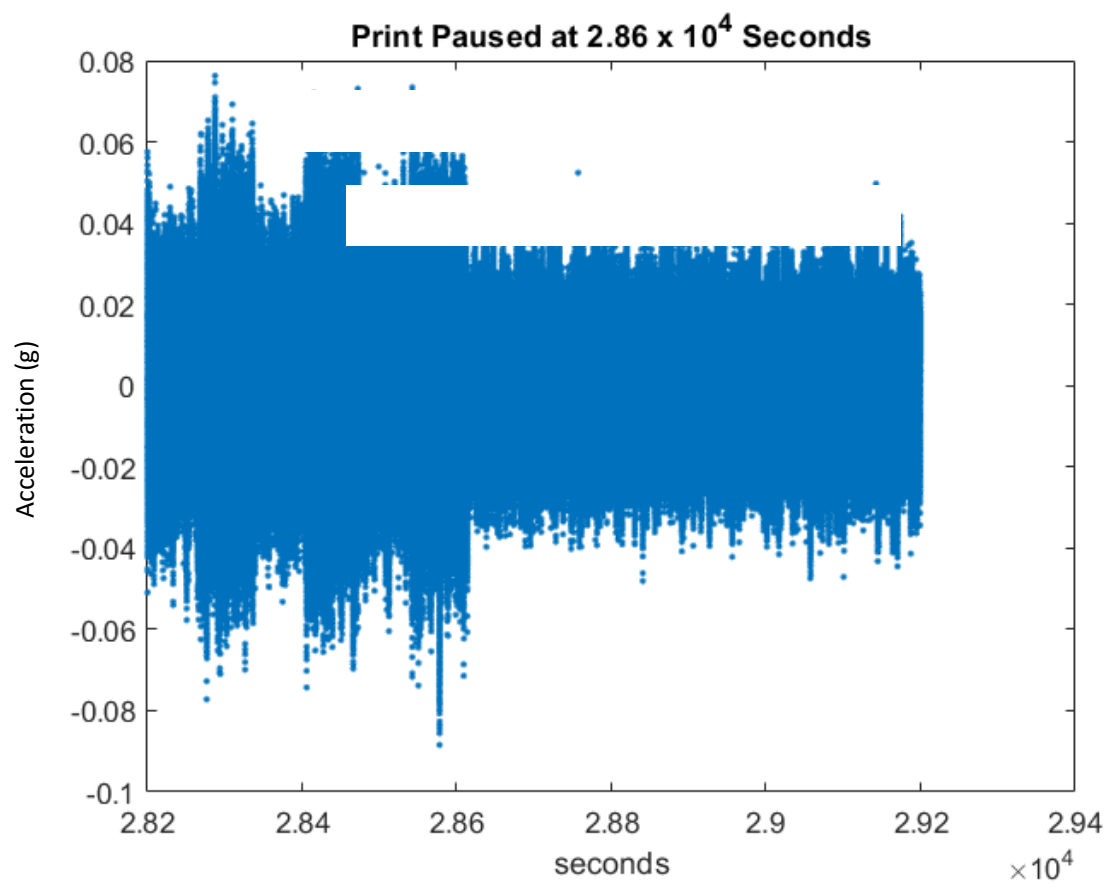

Figure 42. A closeup of the moment the machine is paused reveals a decrease in the magnitude of the accelerometer noise. The periodic behavior of the later recoater section is lost and random noise fills the accelerometer channel.

For characterizing the machine background noise in the frequency domain, discrete Fourier Transform was chosen. Fourier Transform is ideal for signals that are continuous over an extended period. The Fourier Transform demonstrates the hum of the machine when it is on is centered around $700 \mathrm{~Hz}$. In comparison, the machine off noise is scattered through a range of difference frequencies (Figure 43). In addition to Fourier Transform, a Continuous Wavelet Transform was conducted to see the results of the frequencies over time. In the wavelet decomposition, the magnitude of the signal is much weaker than that 
in the latter recoater region and the magnitude of the frequencies are spread throughout the spectrum more evenly.

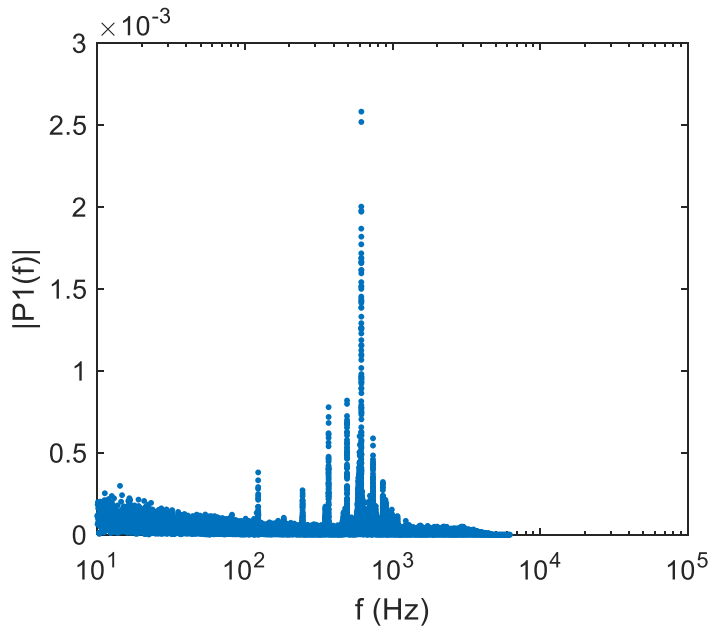

a

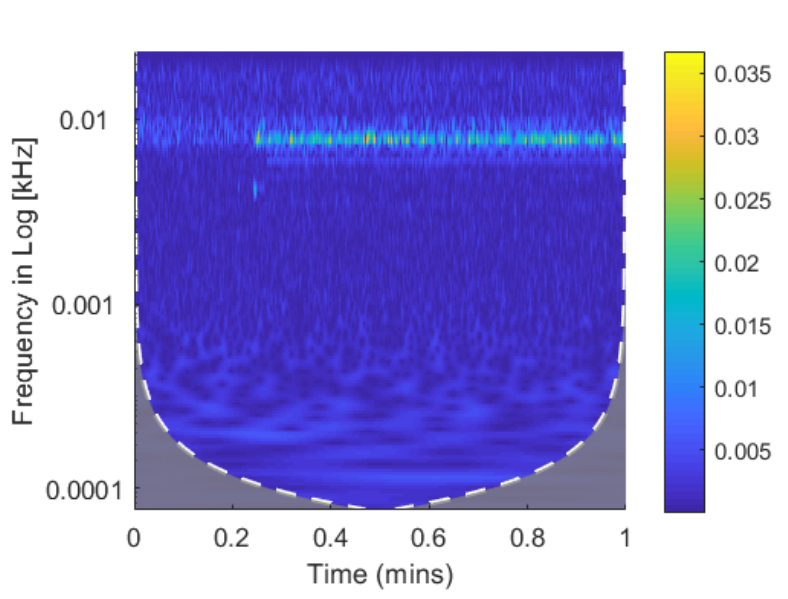

$\mathrm{C}$

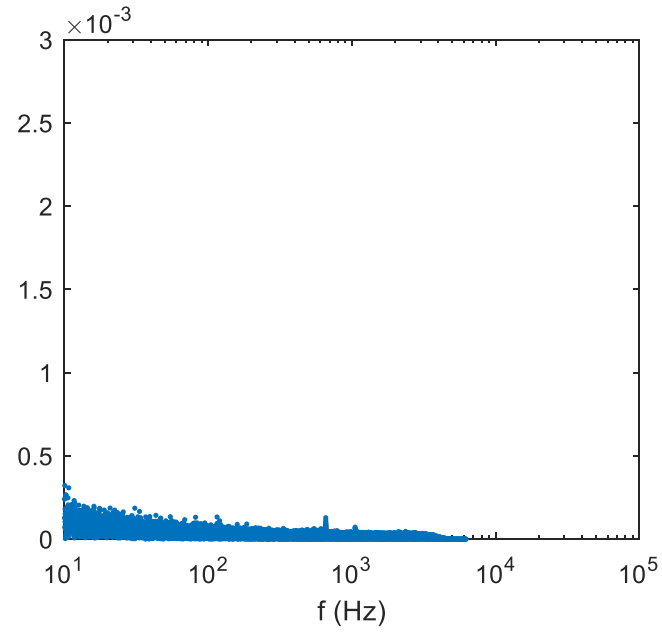

b

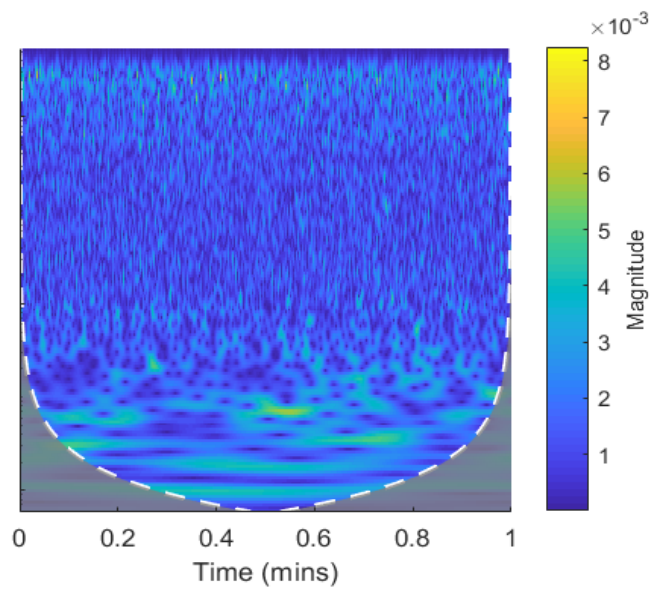

d

Figure 43. Fourier Transforms of the Machine On (a) to Machine Off (b) show the more prominent pattern in frequencies the Machine On. The Wavelet Decomposition of Machine On (c) also has a prominent hum at $700 \mathrm{~Hz}$ that is missing from the Machine Off plot $(d)$. 


\subsubsection{Warpage Defect Signature}

Warpage is characterized in the accelerometer data by large recoater spikes surrounded by additional noise. The recoater spikes correlate with machine script commands for recoater movement. The magnitude of the recoater spikes are inconsistent in this region of the build (Figure 44). The variation in amplitude of the spikes is due to the changing height of the warpage between layers, as well as the recoater movement direction. If the warpage is sharper in one direction, the recoater may tear, creating a higher acceleration.

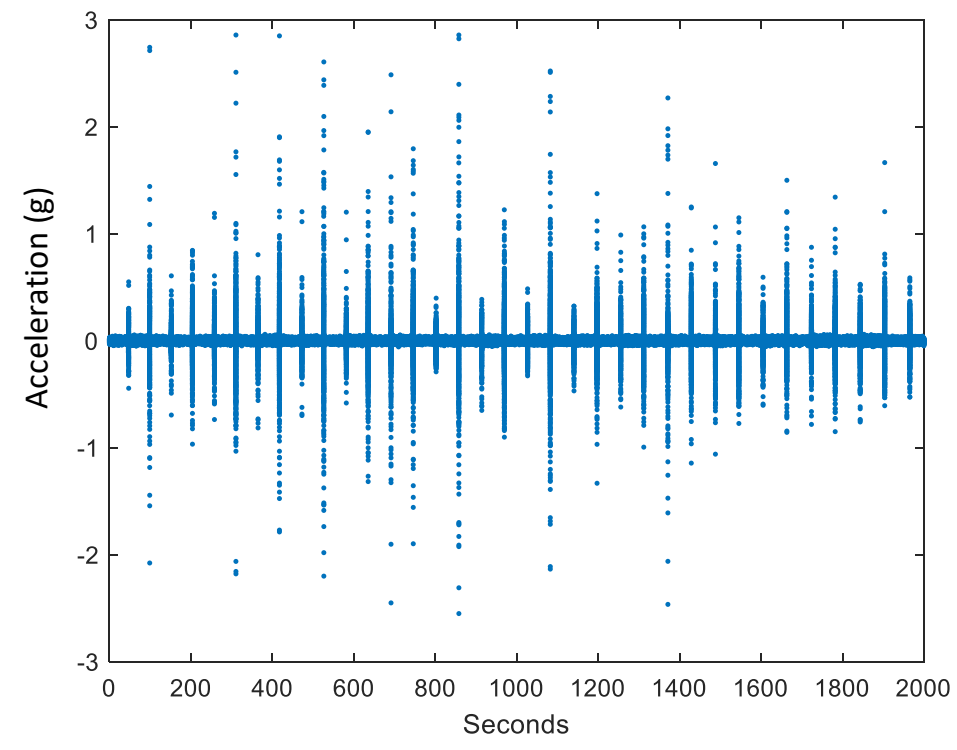

Figure 44. A close up of the accelerometer versus time plot during warpage. The warpage creates a variety of different recoater spikes that are inconsistent in amplitude.

Surrounding each large recoater spike is additional noise that is produced as the recoater blade moves across different areas of warpage on the build plate (Figure 45). The large spike occurs when the recoater hits into an extremely high section of warpage. During 
this contact, the recoater blade may tear, creating a large acceleration. Other areas that have less warpage will result in a smaller acceleration.

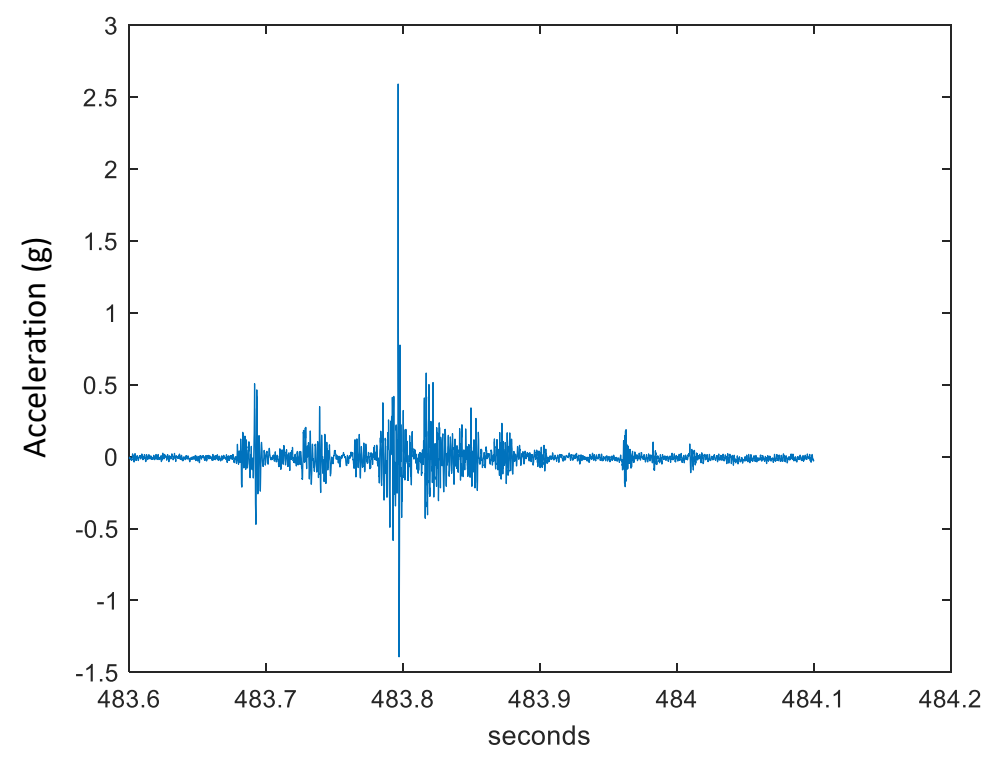

Figure 45. A closeup of the recoater movement in the warpage section of the build is characterized by large amplitudes when the recoater blade contacts sharp or severe warpage sections and noisy smaller spikes around the main spike as the recoater runs over lower warpage areas.

When the large recoater spike is observed up close, a single large upward and downward peak are seen (Figure 46). There is no exponential decay envelope as would be expected in a sudden free shock vibration, like a crack. When the recoater blade hits the warpage, it creates a sudden large spike. Since the recoater is still in contact with the build plate, however, the movement is damped out quickly. Essentially, the system is overdamped when the recoater hits the warpage so no free vibration pattern results. 


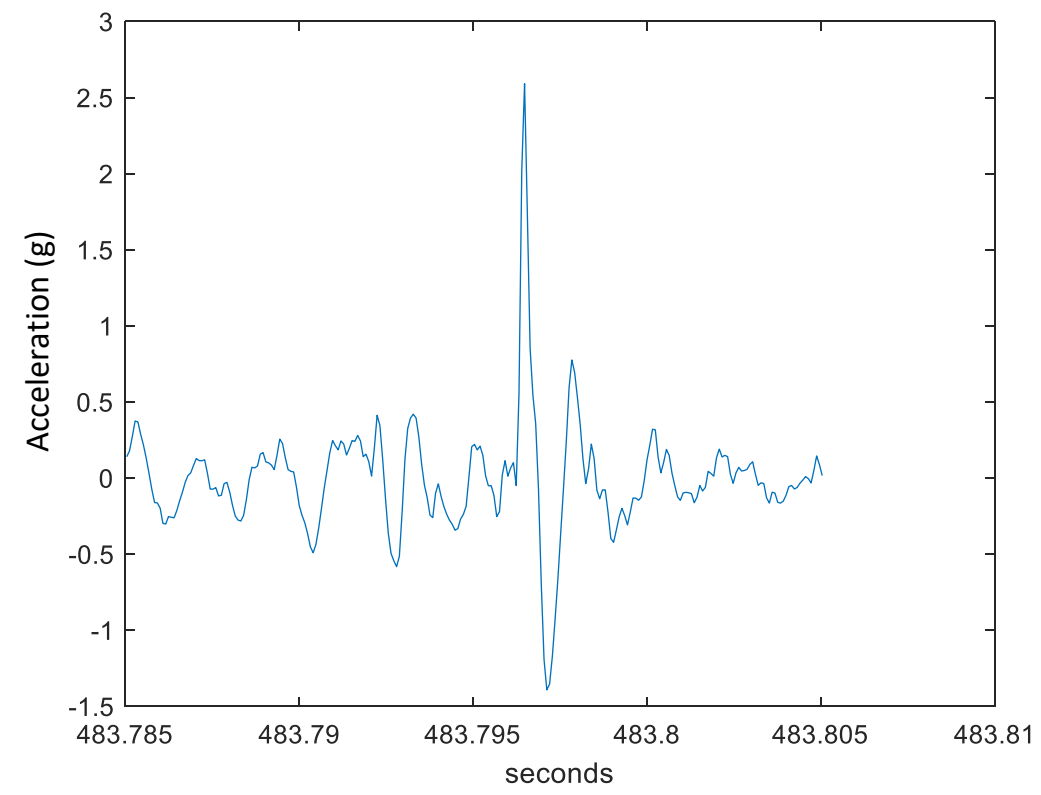

Figure 46. The acceleration versus time plot of the large recoater spike does not exhibit free vibrations implying that it is overdamped. This damping could be due to the position of the recoater blade on top of the build plate during the spike.

In the frequency domain, the spike is characterized by a lower frequency (Figure 47). The recoater movement cannot energize the higher frequencies because the recoater movement is a step input, rather than an impulse. The acceleration is from the recoater rubbing and ripping rather than a sharp hit. The recoater movement in the warpage section has a high magnitude, however, the form of the recoater signature is not appropriate for exciting higher frequencies. 


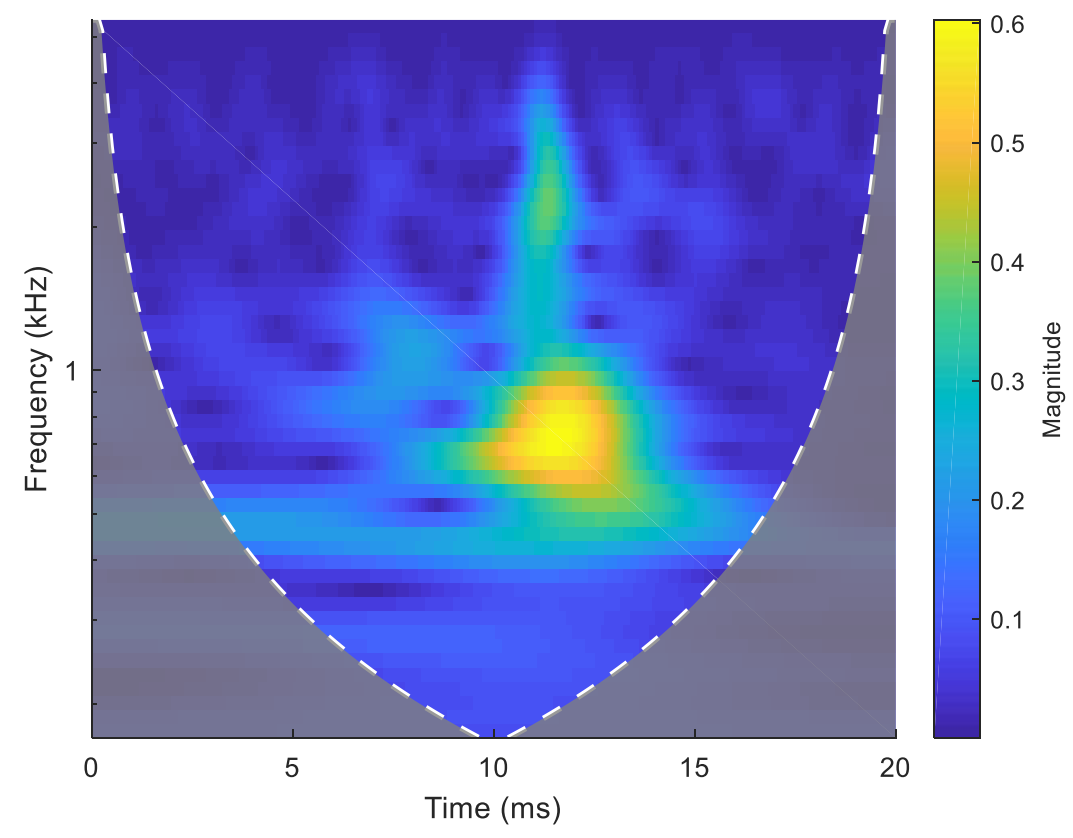

Figure 47. The Wavelet Decomposition plot demonstrates that while the Warpage spike has a large magnitude, it cannot excite higher frequencies.

\subsubsection{Cracking Defect Signature}

A crack in the narrow leg portion of the test coupon is caused by the shrinking of the upper large mass. The leg is not strong enough to resist stress caused by the mass's movement. After approximately $15 \mathrm{lbs}(68 \mathrm{~N})$ of force, the leg is broken in a tensile failure. The force of this failure is experienced by the accelerometer as an impulse. Three potential crack events were identified in the accelerometer data for only one test coupon. The test coupon is titled build 0056 and was printed using the chess parameters. These events were discovered because they have much higher amplitude than their surrounding signal data (Figure 48). The second and third potential crack events in the accelerometer data occurred within 0.03 seconds of each other, indicating that the second and third crack events may be related. 

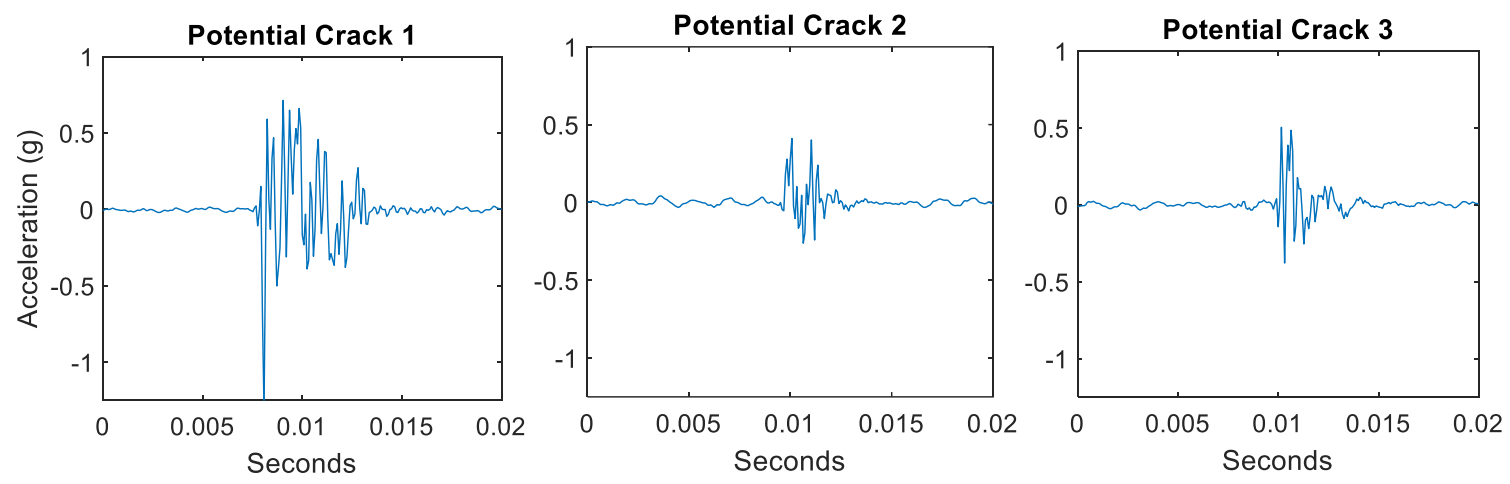

Figure 48. Three acceleration versus time plots indicate there were three potential crack events captured by accelerometer data during the cracked build 0056.

\subsubsection{Evidence to Support Cracking Event}

The presence of the cracks in the part and evidence of a shift in part location during the build, provide physical evidence to support the occurrence of three potential crack events in the accelerometer data. From visual inspection, build 0056 had two completely cracked legs in the thin $\mathrm{h} \_15$ test coupon (Figure 49). One of the cracks is V shaped, signifying the crack may have formed in two different times. This could align with the second and third accelerometer spikes that occurred close together in time. 

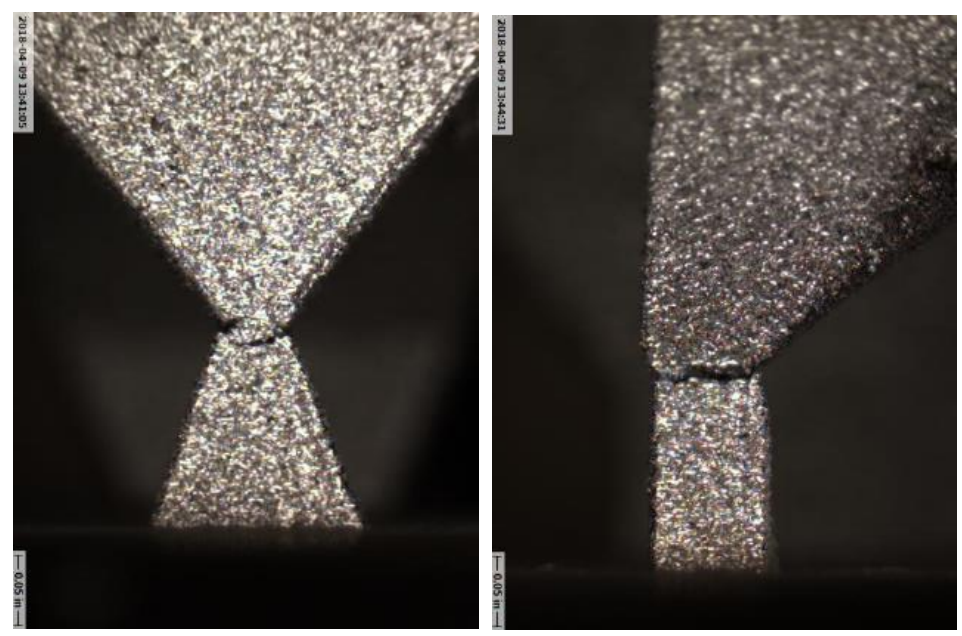

a
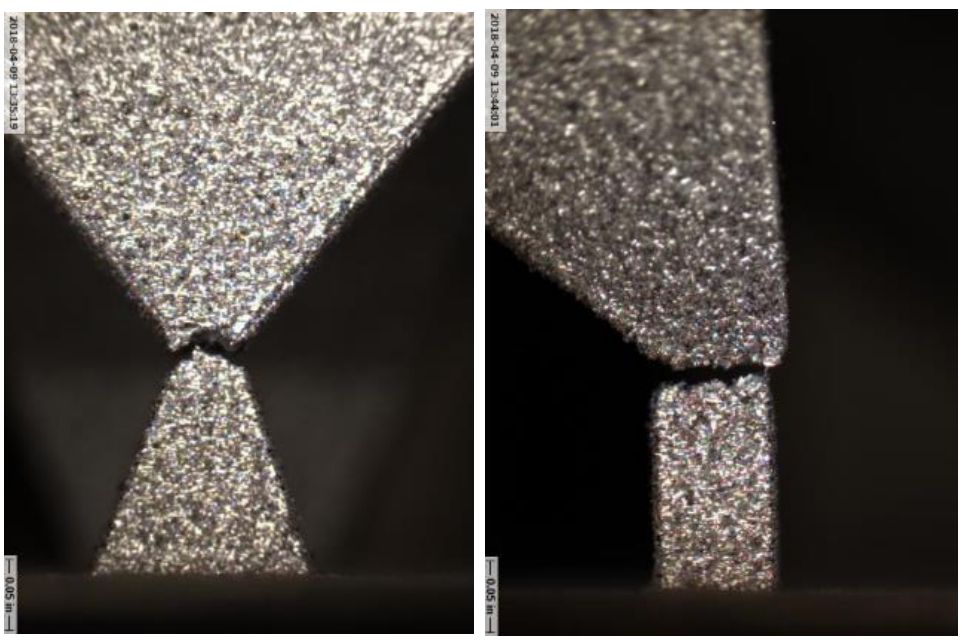

b

Figure 49. The cracks in the left leg (a) and the right leg (b) of the test coupon are viewed using a MicroVu.The crack in the right leg $(b)$ is $v$ shaped. This crack shape may be formed by two separate crack events.

In addition to cracks present in the finished part, there is physical evidence that the powder bed was shifted around the same layers that potential crack signatures appeared. The printer does not assess part location during a build. If a part moves during the build, 
the following layers are printed in the same absolute position. In this way, a part will display a shift line where the top section of the part is at a slightly different location than the bottom section (Figure 50). Unfortunately, build 0056 was paused for a machine maintenance warning between the first and second potential cracking spikes. Due to this event the shift line could be either due to part cooling and shrinking during the pause or due to a sudden shift caused by a crack. Opportunely however, the previous build completed at LLNL on the Concept Laser machine contains the same signature line at around the same location in the test coupon. In addition, the shift moved the test coupon in a single direction. If the shift was induced by cooling, it should show the test coupon shrinking inward instead. Thus, overwhelming evidence suggests that the cracks did occur during the same section of layers that the accelerometer data contains irregular spikes.

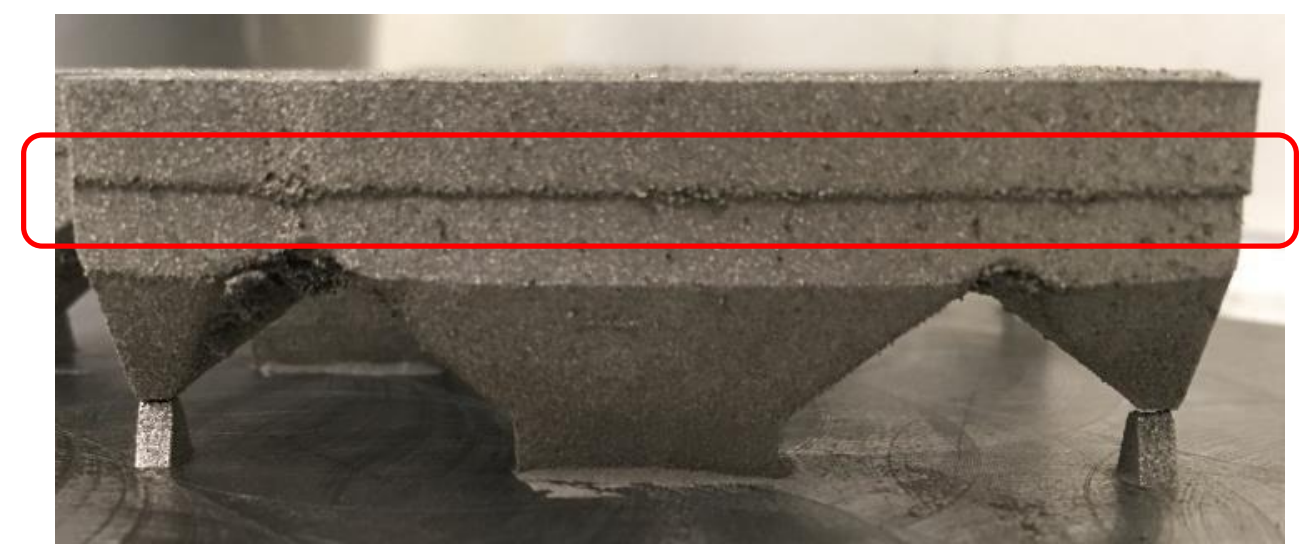

Figure 50. A shift line seen in the photograph of the test coupon occurs when the test coupon moves during the build and the printer continues to print as if the test coupon has not moved. 


\subsubsection{Characterization of Cracking Signal}

The crack signals have characteristics in both the time and frequency domains that suggest they are an impulse event. Unlike a noisy recoater spike, the crack spike has smaller vibrations only on the trailing side of the event (Figure 51). These smaller vibrations make up the shock envelope and can be characterized by their frequency and rate of decay.

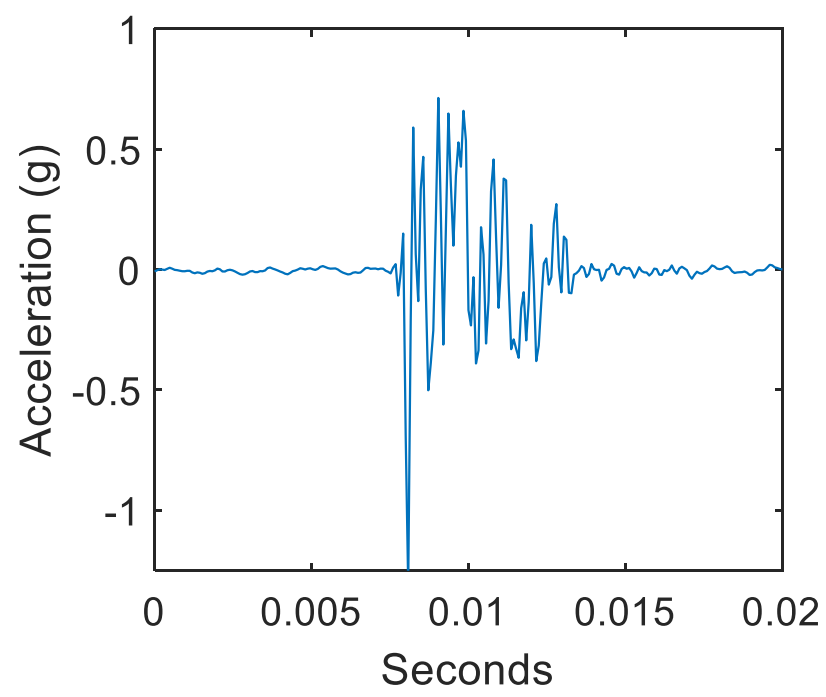

Figure 51. A snapshot of a crack is distinguishable by its sudden large amplitude and its one-sided rate of decay.

Using a linear model for decay, the rate of the decay is max height divided by time elapsed from max height to normal operating amplitude. The linear rate of decay for the three potential cracks was $0.005,0.009$, and $0.005 \mathrm{~g} / \mathrm{sec}$. The rate of decay is affected by the structure of the test coupon as well as the depth of powder when the crack occurs. These factors affect the damping in the system. It is expected that different test coupons would have different rates of decay. 
A rule developed through this project is that a spike is only considered a potential crack if its amplitude is significantly larger than the surrounding signal's amplitude and its duration is less than 0.5 seconds. This definition includes events like recoater movements as potential cracks. For the following discussion, however, a potential crack is defined by an isolated high amplitude event that is not easily identified as a recoater movement due to its location in the accelerometer profile and the lack of a corresponding timestamp in the machine script. To determine what signifies a crack from other isolated high amplitude events, potential cracks were analyzed alongside two verified events: recoater movements and modal hammer tests. It was found that the modal hammer and potential crack events shared the same profile of a large spike followed by a decaying amplitude of smaller spikes (Figure 52). The recoater spike, however, had small vibrations before and after a single large spike.

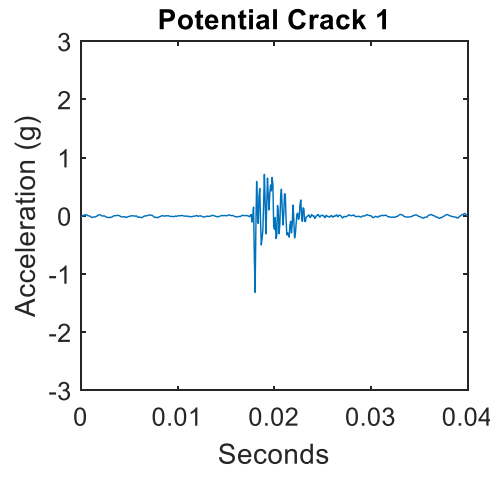

A

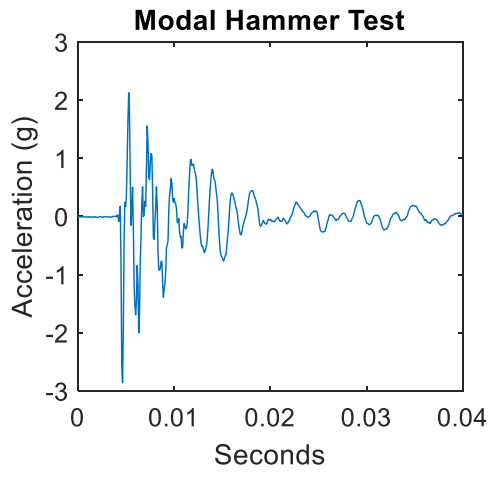

b

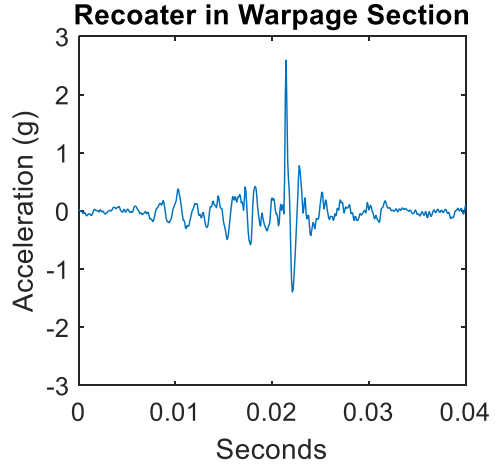

c

Figure 52. Acceleration versus time plots of the Crack (a) and the Modal Hammer (c) shared similar profiles compared with the Recoater Movement (b).

When the leg cracks it causes a sudden shock in the system, followed by free vibration. The signal of the crack was separated from the surrounding machine noise signal through analysis in the frequency domain. Wavelet Decomposition and Shock Response 
Spectrum were both attempted, however, only wavelet decomposition proved successful in this situation due to sample rate limitations. The accelerometer data contains on average five data points per peak (Figure 53). While the sample rate is acceptable, it is the lowest threshold needed for accurate analysis. Low sample rates can lead to aliasing in the dataset. Aliasing was discussed in section 4.1.1: Experimental Set Up.

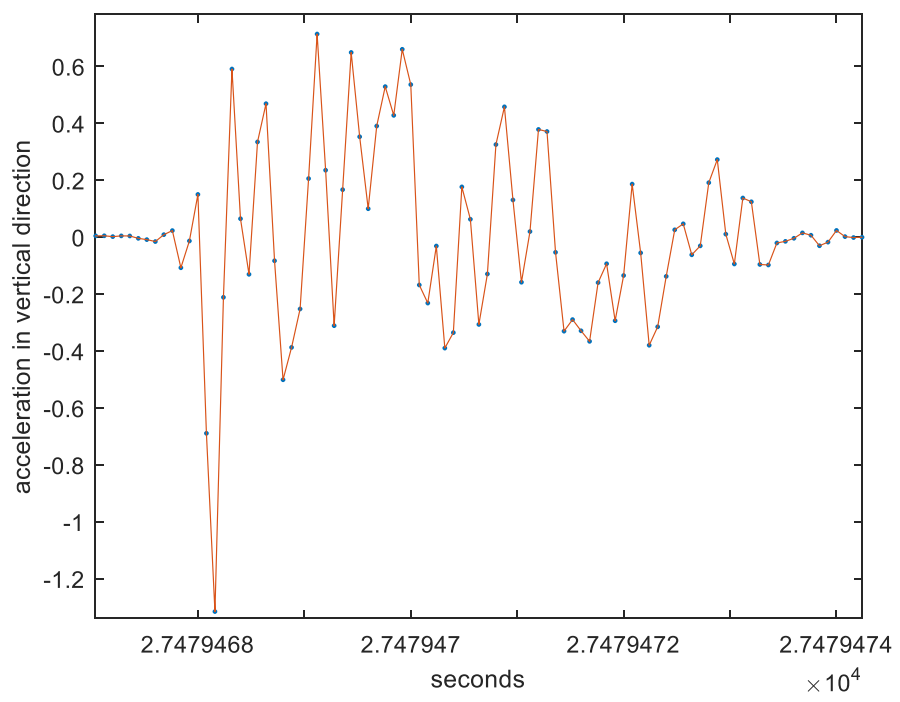

Figure 53. An Acceleration versus time plot shows there is an average of five data points per peak, which is low but acceptable in signal analysis.

The three potential crack events were displayed on a frequency versus time plot using Matlab's cwt.m function. The height and width of the shape of the three events were the same (Figure 54). All three events excited patches of frequencies throughout their shape, rather than all the frequencies in the range (Table 6). All three cracks contained higher frequencies at the large spike and lower frequencies before and after the event. 


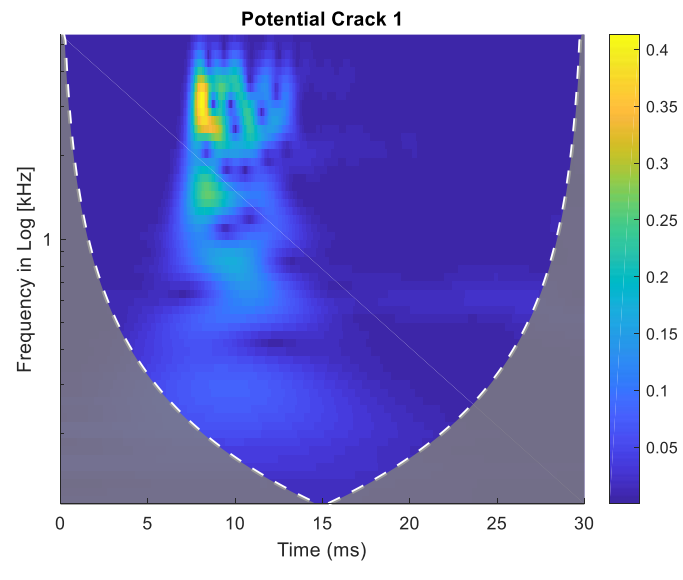

$a$

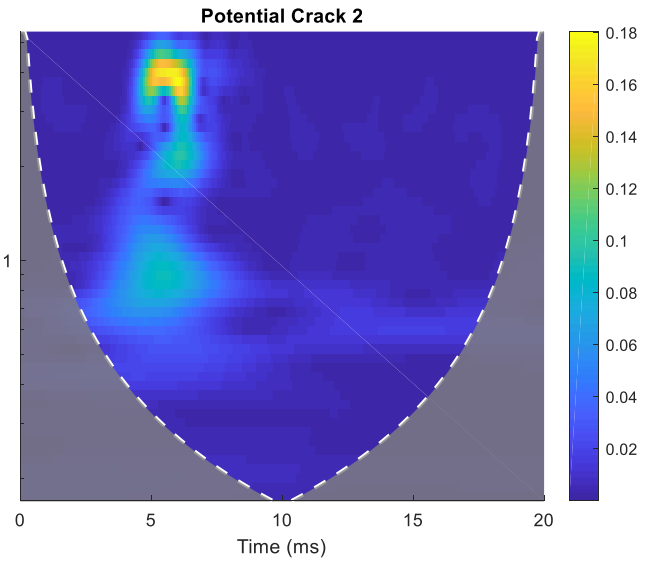

$b$

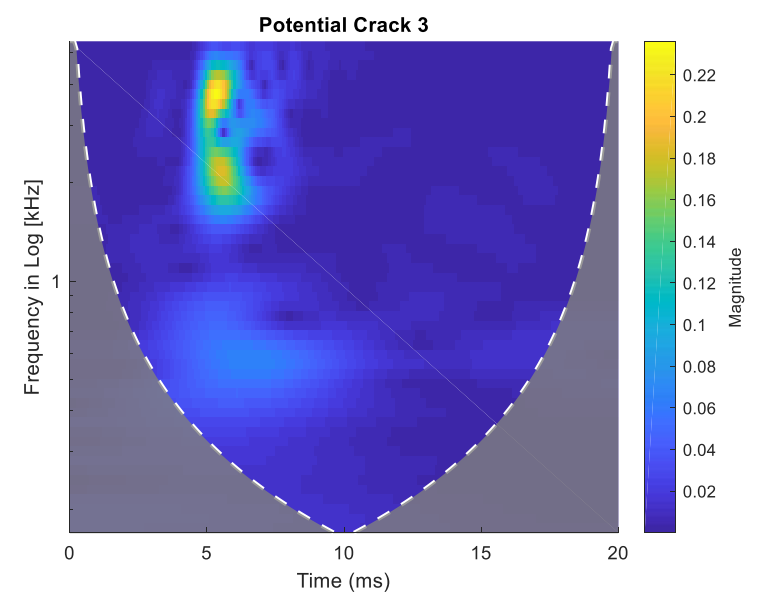

$c$

Figure 54. The frequency versus time plot of the three potential cracks had the same overall shape. 
Table 6. The frequencies excited by the three potential cracks are summarized in the table. The frequencies were selected as the center of the hot yellow spots on the wavelet plots.

\begin{tabular}{|c|c|}
\hline Frequencies Excited (Hz) & Potential Cracks \\
\hline 590 & 3 \\
\hline 835 & 1,2 \\
\hline 1450 & 1 \\
\hline 2204 & 2,3 \\
\hline 3100 & 1 \\
\hline 3837 & 2,3 \\
\hline
\end{tabular}

The crack's frequency response was then compared to the known events of a recoater's movement during warpage and a modal hammer test. While the modal hammer test is not a usual print noise, it was used as an impulse comparison. After performing Wavelet Decomposition, the modal hammer and the crack events were seen to have a more similar profile than the recoater event (Figure 55). The recoater event had a large magnitude in the lower frequencies, while the hammer and the crack had larger magnitudes in higher frequencies. Few repeated frequencies were found between the hammer, recoater, and cracking events (Table 7). The limited repeated frequencies are partially due to the lack of a complete modal hammer test. 

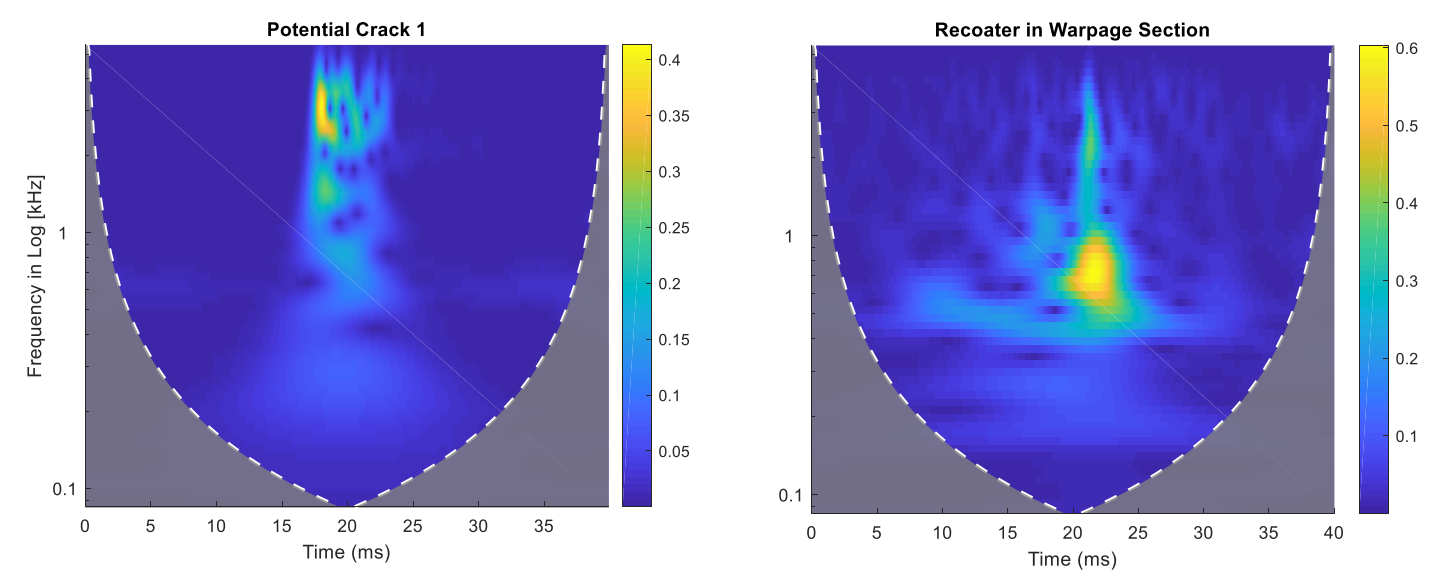

A

b

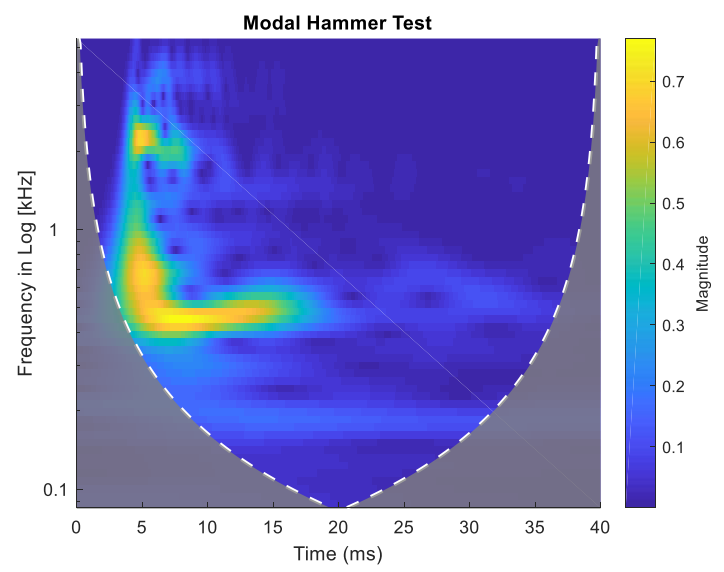

$\mathrm{c}$

Figure 55. The frequency versus time plots of the crack (a) and the modal hammer test (c) both contained higher frequencies than the recoater movement (b). 
Table 7. The frequencies excited by various events are summarized below for comparison. The cracks $(\mathrm{C})$ have higher frequencies in general than the hammer $(\mathrm{H})$ and recoater $(\mathrm{R})$.

\begin{tabular}{|c|c|c|c|c|c|c|c|c|c|}
\hline Frequency (Hz) & 447 & 590 & 678 & 727 & 835 & 1450 & 2204 & 3100 & 3837 \\
\hline Event & H, R & C3 & H & R & C1, C2 & C1 & C2, C3, H & C1 & C2, C3 \\
\hline
\end{tabular}

While all three events are large in magnitude, only the impulse events of the modal hammer and the crack were able to excite higher frequencies above the machine's operating frequency of $700 \mathrm{~Hz}$. To excite higher frequencies, an event needs to contain a lot of energy in a relatively short period of time. The recoater event occurred more slowly and its energy was dispersed over the build plate unlike the cracking and the modal hammer events. In addition, the recoater event took place while the recoater was on top of the build plate, further dampening it. While the crack event was the smallest in amplitude, it was able to excite the highest frequencies (3100 and $3837 \mathrm{~Hz}$ ). This excitation implies that the impulse from the crack had more energy than the impulse from the modal hammer. As the hammer used for the modal hammer test was a dead blow hammer designed to dampen out high frequencies, it is reasonable that the impulse of the hammer did not excite as high of frequencies as the crack.

In summary, there were three potential crack signals identified in the build due to their high amplitude. The time of these signals align with a shift in the powder bed and the presence of cracks in the part. The profile of their signal represents an impulse event. In the frequency domain, the potential crack events can excite higher frequencies than either a modal hammer test and a recoater movement. These frequencies, however, need to be 
verified with more data taken at a higher sample rate. The low data collection rate makes analysis in the frequency domain unreliable, especially for frequencies greater than $1 / 10^{\text {th }}$ of the collection rate, or $1250 \mathrm{~Hz}$.

\subsubsection{FEA Modal Frequency Simulation}

A Solidworks Simulation was used to determine the anticipated modal frequencies for the structure. For the FEA model, an assembly of a build plate and three test coupons was created using stainless steel 316 properties. The build plate was constrained at the four bolt holes and the test coupons were rigidly connected to the build plate. The three modal frequencies were approximately 2700,4700 , and $5000 \mathrm{~Hz}$. The modal shapes were bending and twisting of the three different test coupons when one leg is cracked is shown below (Figure 56).

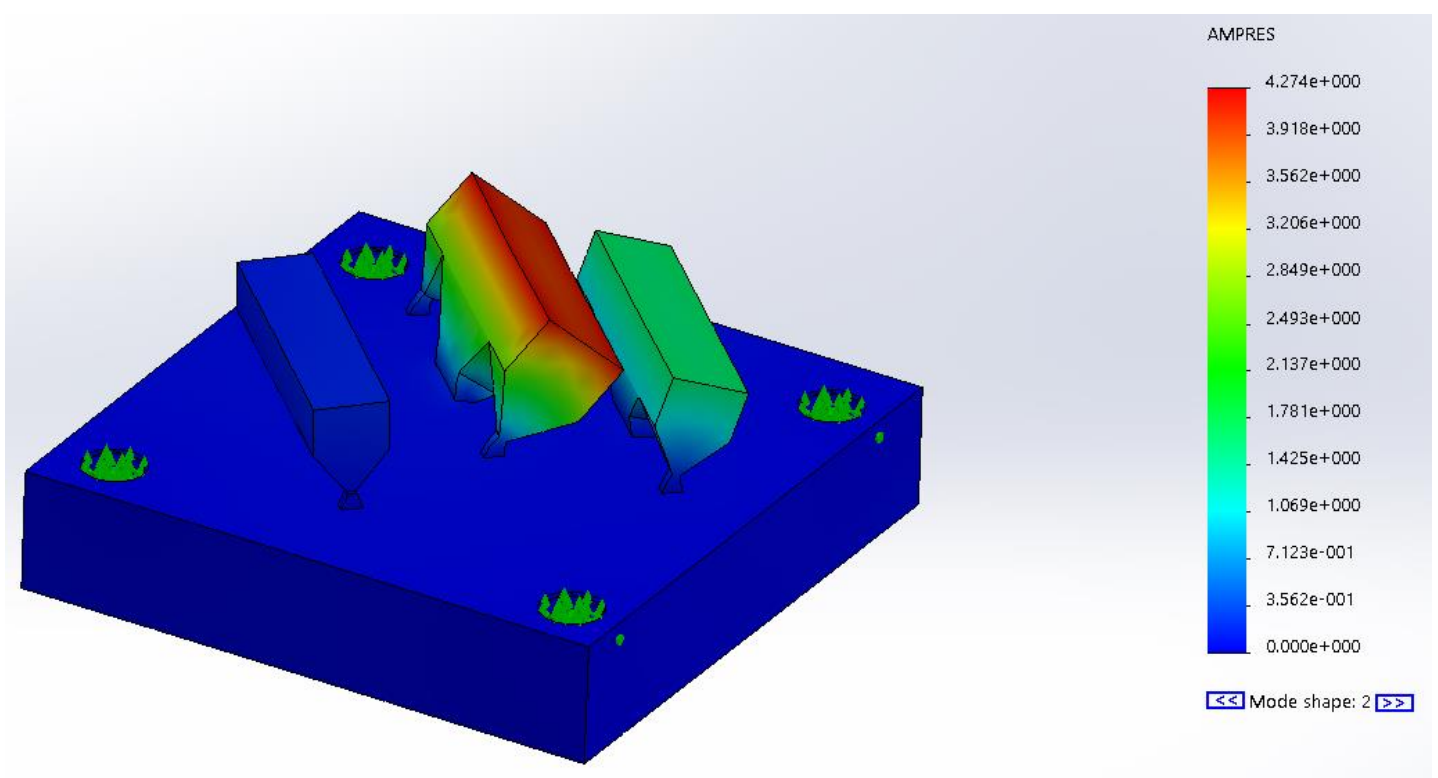



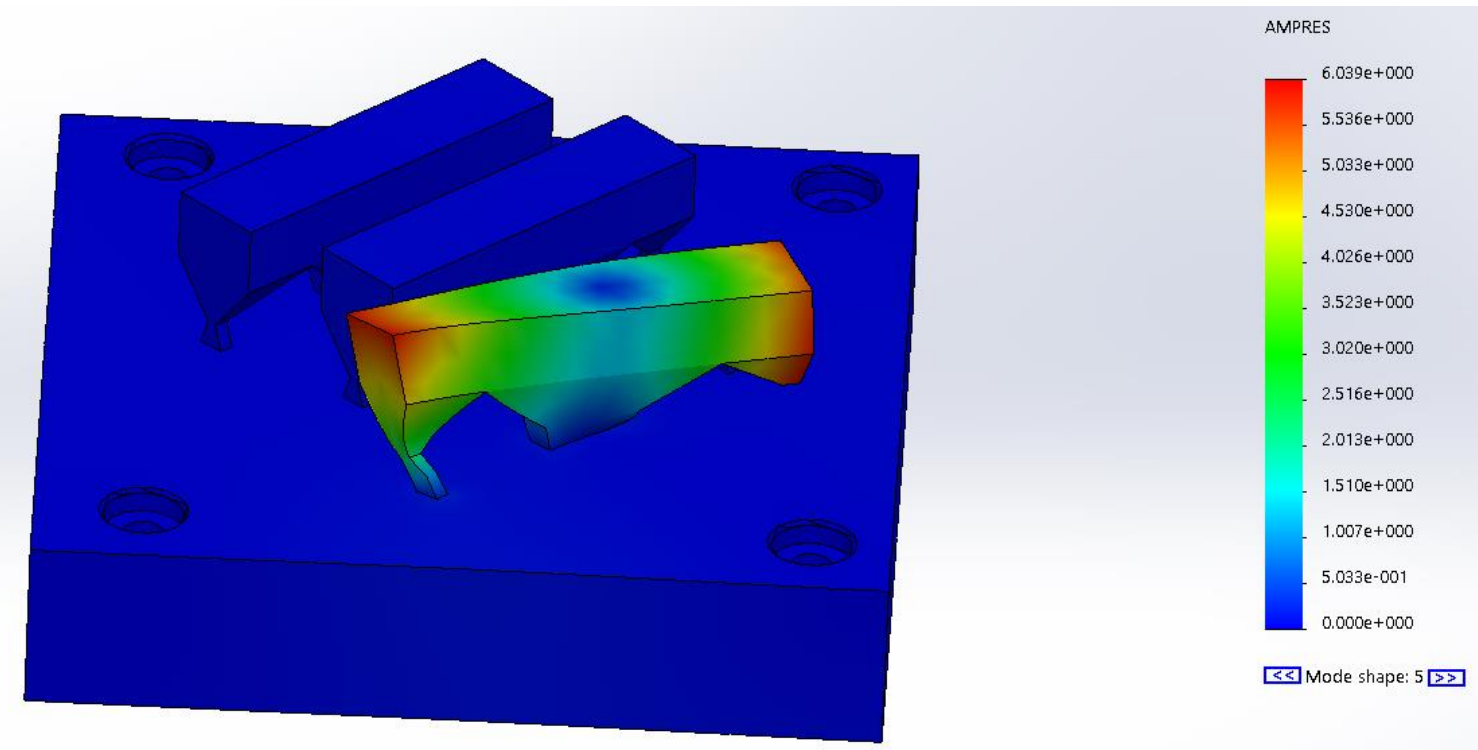

$\mathrm{b}$

Figure 56. The modal shape for $2744 \mathrm{~Hz}$ is shown in the Solidworks Simulation (a). The higher frequency of $5140 \mathrm{~Hz}$ produces a twisting mode shape of the parts $(b)$.

Variations of the model included shifting the position of the parts and creating a crack in the legs of the parts. The modal frequencies did not change dramatically based on the test coupon location or whether the legs of the test coupons were broken. When one leg on all three test coupons were broken, the natural frequencies decreased slightly with the first natural frequency, for example, being $2600 \mathrm{~Hz}$. The mode shapes also changed as the test coupons had less connections with the ground. The FEA model of the one cracked leg approximately matched the physical test results from the two later crack events -2200 and $3800 \mathrm{~Hz}$. As the FEA model did not include the rest of the machine, including the leadscrew, it is not surprising that the modal frequencies are slightly different. The FEA model verifies that the frequencies found experimentally by the cracking event and the modal hammer test are reasonable for this structure. 


\subsection{Photo Analysis}

To supplement the accelerometer analysis of the project, photo analysis was used. The SLM Solutions machine includes a built-in camera to analyze powder spread. Photos are taken after each recoater movement to assess the coverage of powder across the build plate. While warpage prevents complete powder coverage, the warpage's surface area in most cases is not large enough to stop the build. In this project, the images were visually inspected to assess warpage and find defects at different stages of the build.

Photos of sequential layers taken in the warpage section were examined to determine if large acceleration is correlated with warpage severity or test coupon movement. A photo's timestamp was used to match the photo with the correct accelerometer data. The accelerometer data was time stamped by comparing the initial recoater movement to the machine script command for recoater movement. It was found that the large recoater spikes occur when the warpage is the most severe and not necessarily when the warpage is moved by the recoater. In addition, it was found that the warpage pieces were moved multiple times throughout the warpage, rather than a single movement. A piece's movement was measured by comparing piece placement in the previous photo using vectors (Figure 57). 

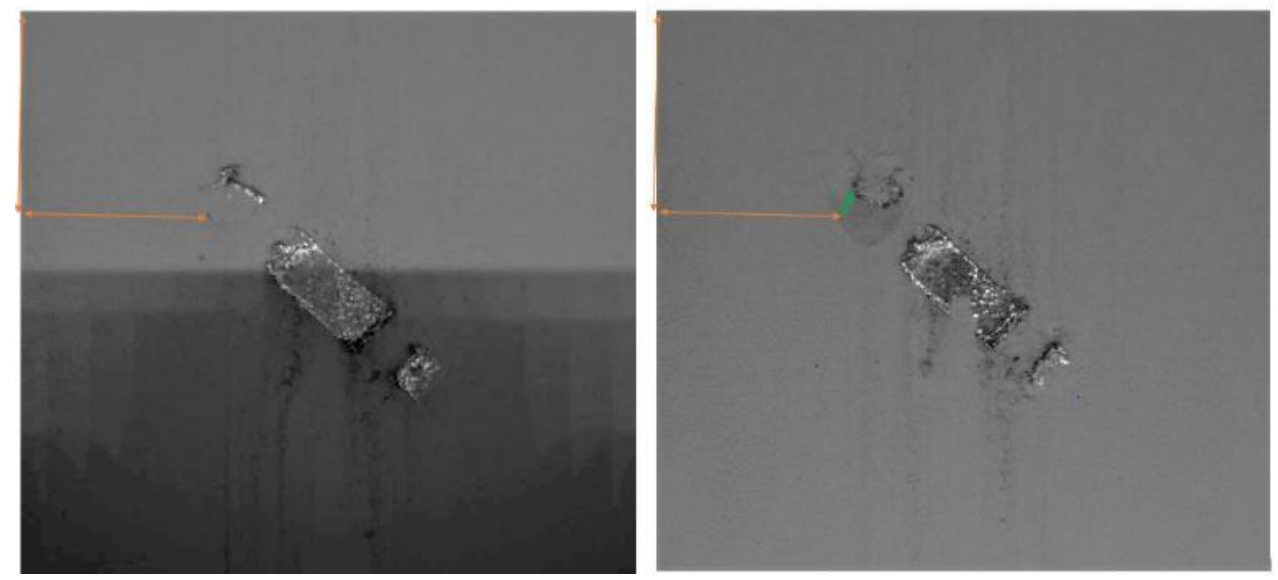

Figure 57. The camera's photos can pick up movements in the test coupon between layers. The green vector (right) shows the difference in test coupon leg placement from the previous layers photo (left).

The photos provided information regarding the warpage defect, however, this technique was not useful in identifying when a crack occurred. The photos are taken after the recoater clears away all evidence of shifting in the layer. Unless the crack occurs in between the time the recoater moves and the photo is taken, a shift due to a crack will not be detected.

\section{$\underline{4.3 \text { Audio Analysis }}$}

The audio was recorded using an iphone. The iphone was positioned on the outside of the door of the build chamber (Figure 58). It was connected to the laptop via an USB cord for charging purposes. The WavePad app was used for recording the data and the Audacity software was used for data processing. The data was recorded at 48,000 Hz. This is four times higher than the $12,500 \mathrm{~Hz}$ sampling rate of the accelerometers. As human hearing is between 20 and $20,000 \mathrm{~Hz}$, the iphone can detect the entire range of human hearing. Due to aliasing issues, however, the iphone can only reliably detect signals correctly of 
up to $4,800 \mathrm{~Hz}$ (1/10 of the recording frequency). This is four times greater than the reputability of the accelerometer data of up to $1,250 \mathrm{~Hz}$.

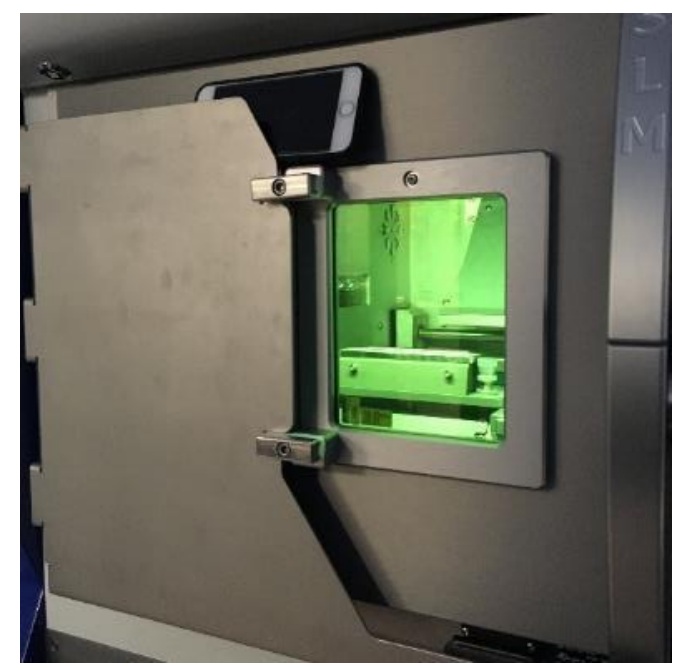

Figure 58. A photograph of the experimental set up shows the iphone was placed on the outside of the build chamber door. It was not rigidly mounted.

A concern with the collection of audio data is the presence of non-related noise signals. A noise test was conducted; showing the audio data is extremely sensitive to external noises. Fortunately, the audio data can be played back to the user to verify unusual spikes. When viewed in the time domain the audio data was consistent throughout the recording, making it difficult to distinguish between areas of warpage and normal operation (Figure 59). There are some louder spikes during the warpage section, but it is not as prominent as in the accelerometer data. 


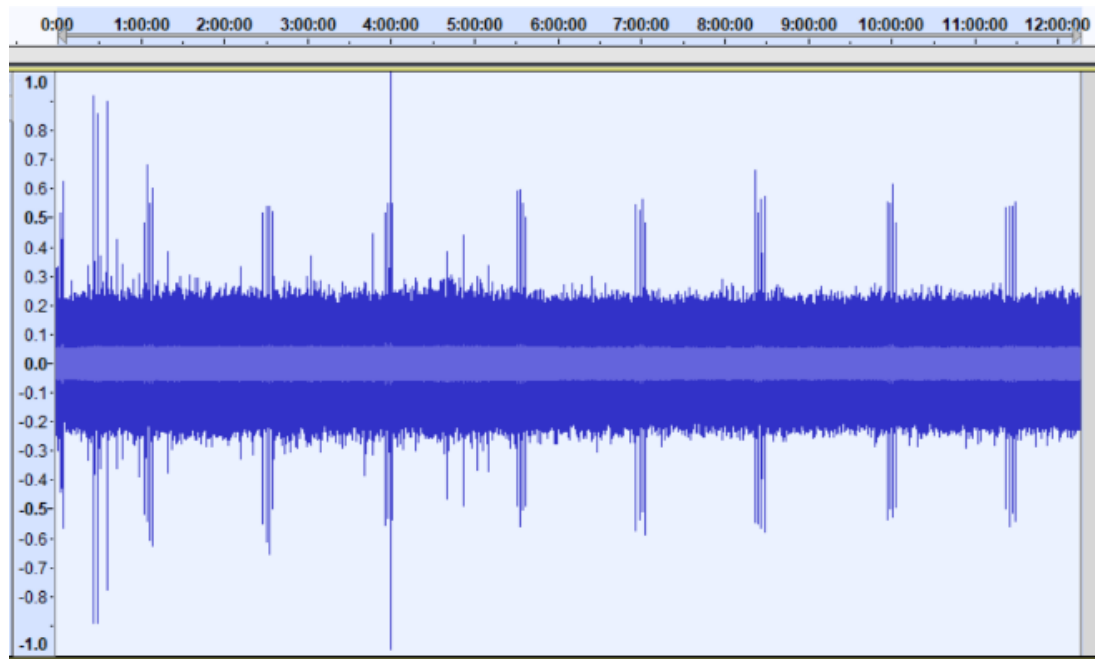

Figure 59. The full audio file on $d B$ versus time indicate it is difficult to distinguish between normal operation and warpage.

When viewed closely, inconsistent spikes are seen in the warpage section of the audio data (Figure 60). These spikes are not easily distinguished from the other noises in the data. In addition, the audio detects loud manufacturing noises such as the release of air in the room every two minutes. This noise is louder than the recoater noises and appears as a long spike in the data set.

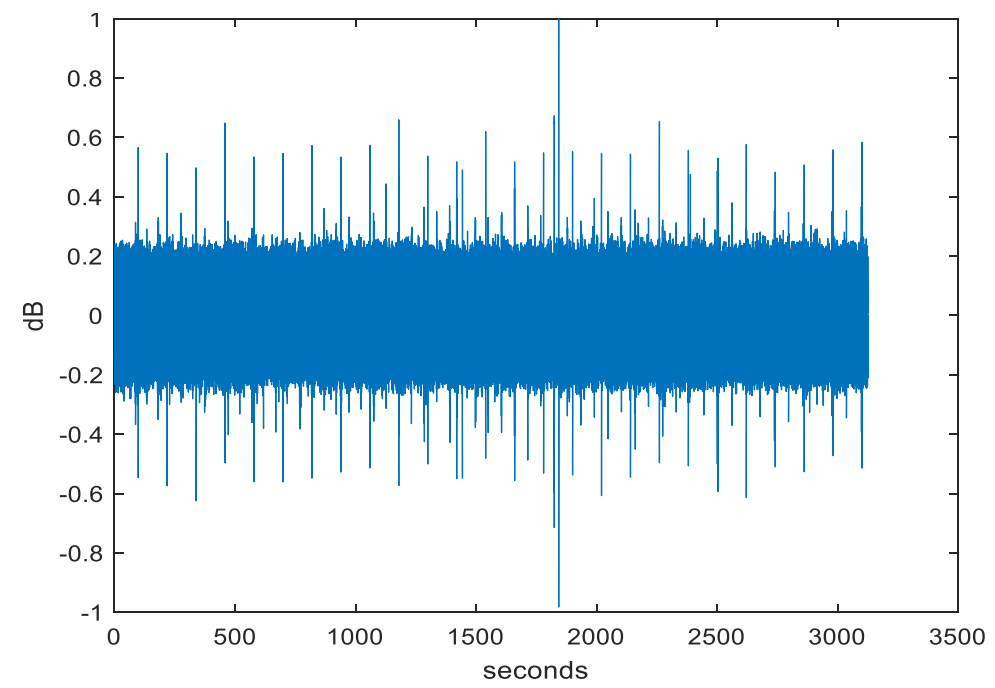

Figure 60. The recoater movements in this Decibel versus time plot of the audio data are not as easily identifiable as the accelerometer data plots. 
Discrete Fourier Transform was used to characterize the machine noise present in the audio signal. The frequencies recorded by the audio are relatively small and are centered about $100 \mathrm{~Hz}$ (Figure 61). These prominent low frequencies are most likely due to the sound of airflow in the room, rather than the building process in the build chamber.

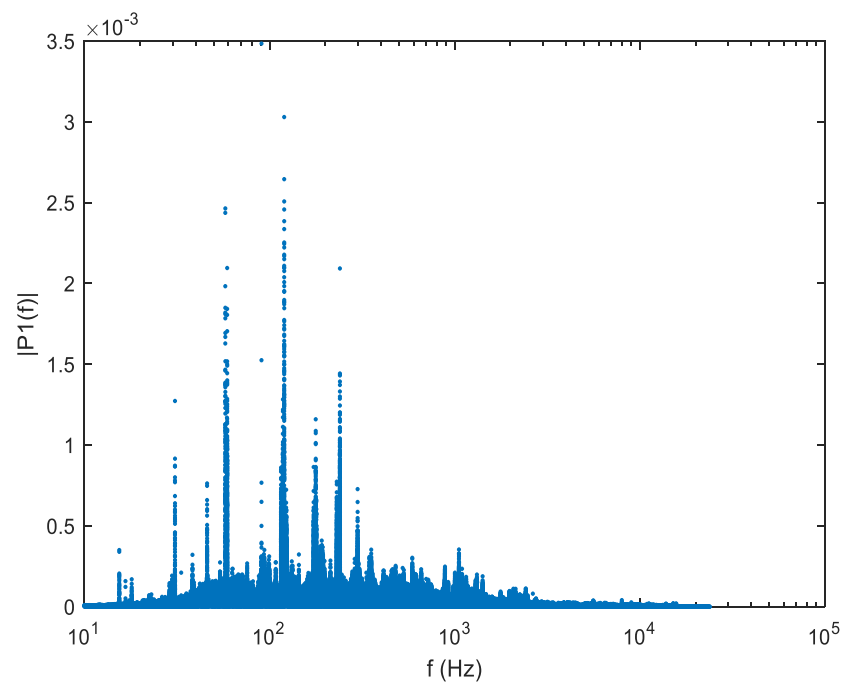

Figure 61. Fourier Transform results indicate that low frequencies are predominant in the audio data.

The purpose of the audio data was to determine if a readily available device with little set up can detect quality of the print. For this reason, an iphone with a free audio recording app was chosen. The lack of quality information from this one audio experiment does not dismiss the use of other more scientific acoustic sensors in the future in detecting part failure. 


\section{$\underline{4.4 \text { Summary of Findings }}$}

Of the three methods used to detect defects, accelerometers were the most effective. Audio and accelerometer data were collected at a higher frequency than image data, making the results more reliable. While photos have valuable information about the failure development that the accelerometers and audio do not contain, the result of detecting a failure is more dependable when using a "continuous" method of data collection. The audio data contains additional background noises that clutter the dataset. In addition, the audio is less sensitive than the accelerometer to changes in the build chamber. While the accelerometer data was the best of the three methods tested, the lack of formality of the audio data means that future data using proper acoustic sensors should be considered.

Accelerometers can detect a crack signature using a sampling rate of $12,500 \mathrm{~Hz}$. The form of the signal produced by the crack is different than the signal created through standard recoater movements. The impulse event appears to produce higher frequencies than normal machine operation, however, these frequencies cannot be verified due to aliasing concerns.

The data in this study is limited and will need to be replicated in the future to verify results. Only one out of ten test coupons printed in the study was successfully cracked. The small dataset prevents the development and testing of a crack finding algorithm to automatically analyze accelerometer data. A larger dataset will allow the patterns found in this study to be further refined for use in machine learning algorithms. 


\section{Chapter 5: Conclusion}

Overall this project was successful in detecting a crack using an accelerometer. Lack of repeatability, however, keeps any signal analysis conclusions preliminary. In the future, more crack data as well as changes to the test coupon are recommended.

\section{$\underline{5.1 \text { Experimental Summary }}$}

In this study, a test coupon was printed on Cal Poly's SLM Solutions Machine ten times. The test coupon, designed by LLNL to crack during the build, features a large upper mass and narrow leg supports. As the upper mass cools, it shrinks causing a tensile normal stress failure in the lower legs. Severe warpage while printing the lower leg portions of the build, however, destroyed the legs before they had a chance to crack as intended in nine out of ten builds.

To limit the severity of warpage, a new set of print parameters were developed called the chess print parameters. These print parameters mimic the printing conditions of LLNL's Concept Laser machine. While prints with the chess parameters were more successful than the prints with the default stripe print parameters, only one test coupon was able to print past the legs without being destroyed.

Accelerometer, Audio, and Image data was collected each build and analyzed on Matlab for irregularities that would suggest defects occurring in the printing process. It was found that the audio data taken on an iphone contained too much background noise and was not able to detect enough variation between good and bad print conditions. The Image data was useful in detecting warpage, however, the timing of the photographs prevent the image data was being useful in detecting cracking. Accelerometer data was 
successful in detecting both warpage and cracking during the build. The accelerometer was sensitive to the rubbing of the recoater blade warpage. It was also able to detect when a crack occurred in the build.

Post print analysis of the accelerometer data pointed to amplitude and frequency as two indicators of cracking. Both cracking and recoater movements have amplitudes six times higher than surrounding machine noise data. To distinguish cracking and recoater movements, wavelet decomposition was used. It was found that cracking can excite higher natural frequencies than the recoater movement. Due to a low sample rate and minimal cracking events, the exact profile of a crack cannot be fully determined from this study alone.

The results of this preliminary investigation into defect detection can be used to create a machine learning algorithm that can monitor a build's quality in real time. The printer could be paused or stopped in an event of catastrophic failure while printing.

\subsection{Future Recommendations}

For future work, the test coupon should be modified to include rounded corners and edges. The sharp geometry increases the possibility of warpage in the part and lead to some browning. Adding fillets on all edges would be an easy method to decrease warpage.

Another recommendation is to increase the accelerometer sample rate. The FIFO error may be avoided by only recording data from the vertical channel. Increasing the sample 
rate will let higher frequencies to be analyzed without the concern of aliasing. There exist more expensive systems that can record high sampling rates for extended periods of time.

\section{$\underline{5.3 \text { Conclusion }}$}

The first part of the project to print a designated test coupon on the SLM by replicating the print conditions on the Concept Laser machine proved difficult as inherent differences between the two machines, such as recoater blade geometry, caused the tearing of the warped legs to continue with the SLM Solutions builds. The one test coupon that had cracks in the legs, served as a single data sample for detecting a crack signature. While there were concerns of aliasing and the dataset was minimal, three distinctive crack events were identified in the accelerometer data. The success and simplicity of the signal analysis makes accelerometers a promising method for defect detection in the SLM process. 


\section{Bibliography}

Budynas, Richard G and Keith J Nisbett. Shigley's Mechanical Engineering Design. New York: McGraw-Hill, 2015. Print.

Cai, Xingfang, et al. "Measurement and characterization of porosity in aluminium selective laser melting parts using X-ray CT." Virtual and Physical Prototyping (2015): 1-12. Pdf.

Carter, Luke N, et al. "The influence of the laser scan strategy on grain structure and cracking." Journal of Alloys and Compounds (2014): 338 - 347. Pdf.

Coria, Angel, Jake Whipple and Shaunessy Grant. Additive Manufacturing Part Failure Detection FDR. Senior Project. San Luis Obispo: California Polytechnic State University, 2017. Pdf.

Devleker, Kirthi. MathWorks: Understanding Wavelets. n.d. Video. 14 February 2018.

Engineering ToolBox. Linear Thermal Expansion. 2008. Web. 27 April 2018.

Fuges, Christina M. Selective Laser Melting in Action. 14 December 2015. Blog.

Irvine, Tom. "An Introduction to the Shock Response Spectrum." 9 July 2012. Vibration Data. Pdf. 10 May 2018.

—. "srs.m." 24 August 2006. Mathworks File Exchange. Code. 15 April 2018.

Materialise. SLM Build Processor. User Manual. Leuven, Belgium: Materialise, 2015. Pdf.

MathWorks. Morse Wavelets. n.d. Web. 20 May 2018.

National Instruments. Understanding and Avoiding NI-DAQmx Overwrite and Overflow Errors. 6 April 2018. Web. 5 May 2018.

Rensihaw. Design for metal AM - a beginner's guide. 7 August 2017. Web. 20 April 2018.

Roberts, Stephen. "Lecture 7 - The Discrete Fourier Transform." 12 May 2003. University of Oxford. Pdf. 15 May 2018.

Sadowsky, John. "THE CONTINUOUS WAVELET TRANSFORM: A TOOL FOR SIGNAL INVESTIGATION AND UNDERSTANDING." Johns Hopkins APL Technical Digest (1994): 306-318. PDF.

SLM Solutions Group. SLM Solutions. n.d. Web. 5 April 2018. 
Society of Manufacturing Engineers. Additive Manufacturing Glossary Definitions. n.d. Web. 5 May 2018.

Wickert, M. "ECE 2610: Introduction to Signals and Systems Lecture Notes." n.d. University of Colorado, Colorado Springs. Document. 15 May 2018.

Yamanaka, Hajime. California Polytechnic State University, San Luis Obispo. Photo. 


\section{Appendix A: Hand Calculations}

Hand Calculations for Bending Stress

1. Force, $\mathrm{T}$, acting on point $\mathrm{c}$ creates moment $\mathrm{M}$ at $\mathrm{a}$.

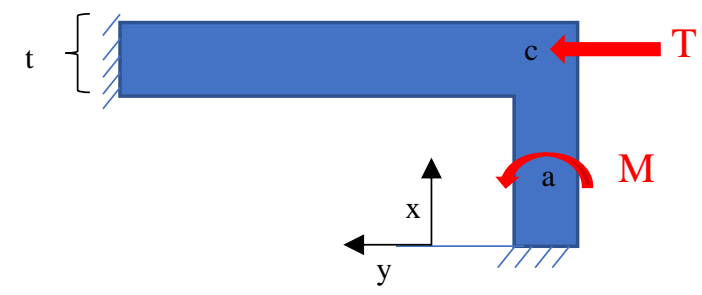

Assume $T \neq w(x)$. Then $M=(c-a) * T$.

$$
\text { Note if } T=w(x) \text {. Then } M=\int_{c-t / 2}^{c+t / 2} w(x)(x-a) d x
$$

2. The effects of the Moment at a cross section at point a.

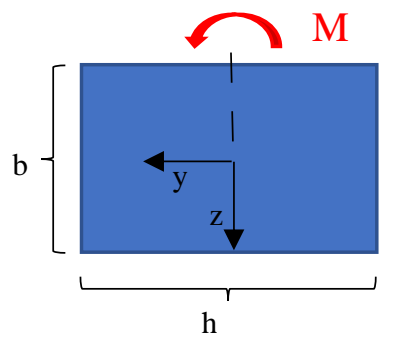

$$
\sigma_{y}=\frac{M y}{I} \rightarrow M=\frac{\sigma_{y} I}{y}
$$

Given for a rectangle cross section: $I=\frac{1}{12} b h^{3}$

$$
M=\frac{\sigma_{y}\left(\frac{1}{12} b h^{3}\right)}{y}
$$


Solving for first moment of fracture at $y=\frac{h}{2}: M=\frac{\sigma_{y} b h^{2}}{6}$

3. Setting $M=M$ from both global and local equations:

$$
\begin{gathered}
(c-a) * T=\frac{\sigma_{y} b h^{2}}{6} \\
T=\frac{\sigma_{y} b h^{2}}{6(c-a)}
\end{gathered}
$$

4. Substituting in values for test coupon (Budynas and Nisbett)

$$
\begin{gathered}
T=\frac{\left(1600 * 10^{6} * \frac{N}{m^{2}}\right) *\left(1.5 * 10^{-3} \mathrm{~m}\right) *\left(2.25 * 10^{-3} \mathrm{~m}\right)^{2}}{6 *\left(c-3.75 * 10^{-3} \mathrm{~m}\right)} \\
T=\frac{900 * 10^{-3} \mathrm{Nm}}{c-3.75 * 10^{-3} \mathrm{~m}}
\end{gathered}
$$

5. For the recoater to tear off the leg: $c=12.25 * 10^{-3} \mathrm{~m}$.

$$
T=106 N \text { or } 24 \mathrm{lbs}
$$

6. To break from thermal stress midway through the upper mass: $c=17 * 10^{-3} \mathrm{~m}$.

$$
T=68 N \text { or } 15 \mathrm{lbs}
$$




\section{Appendix B: Test Coupon Drawings}

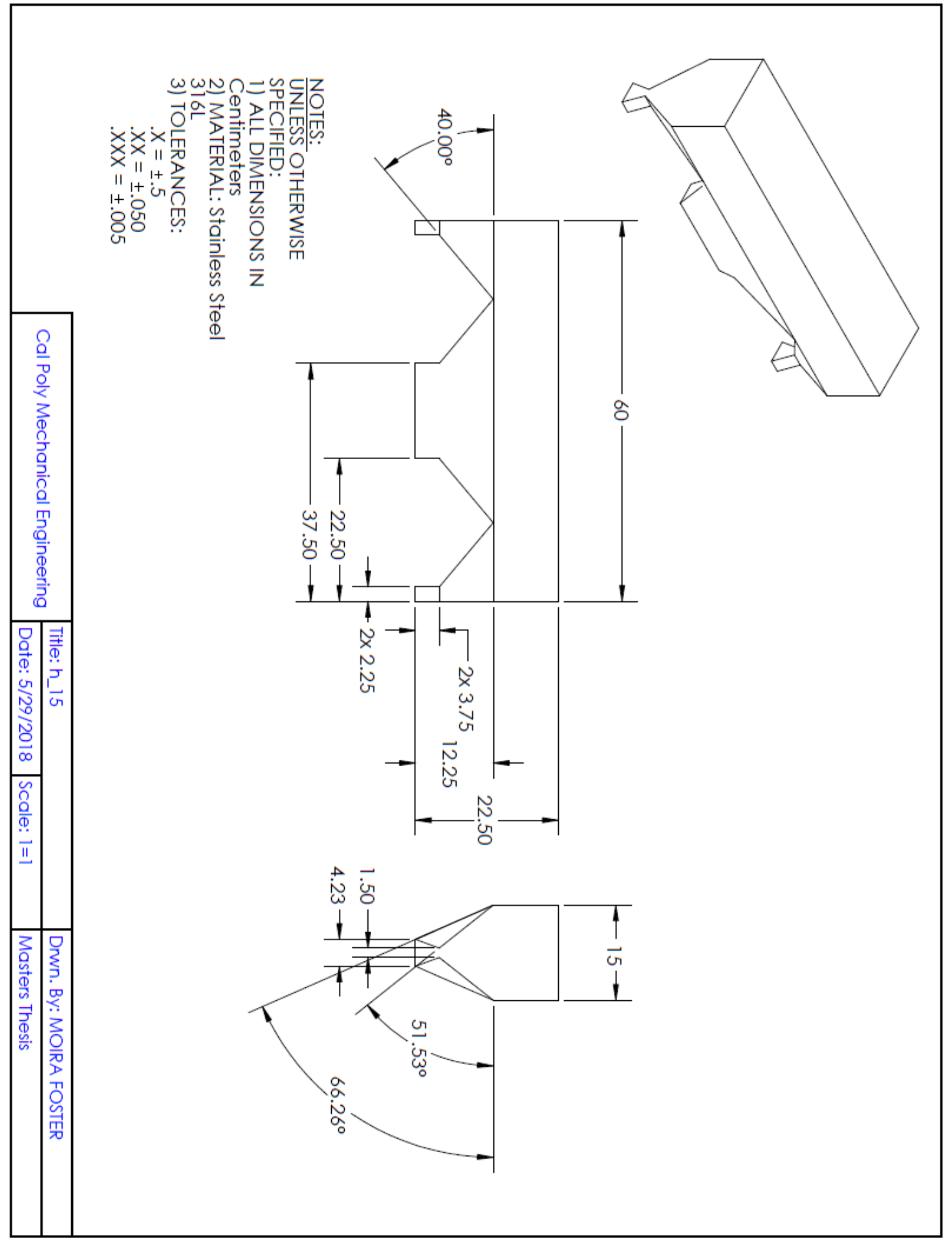




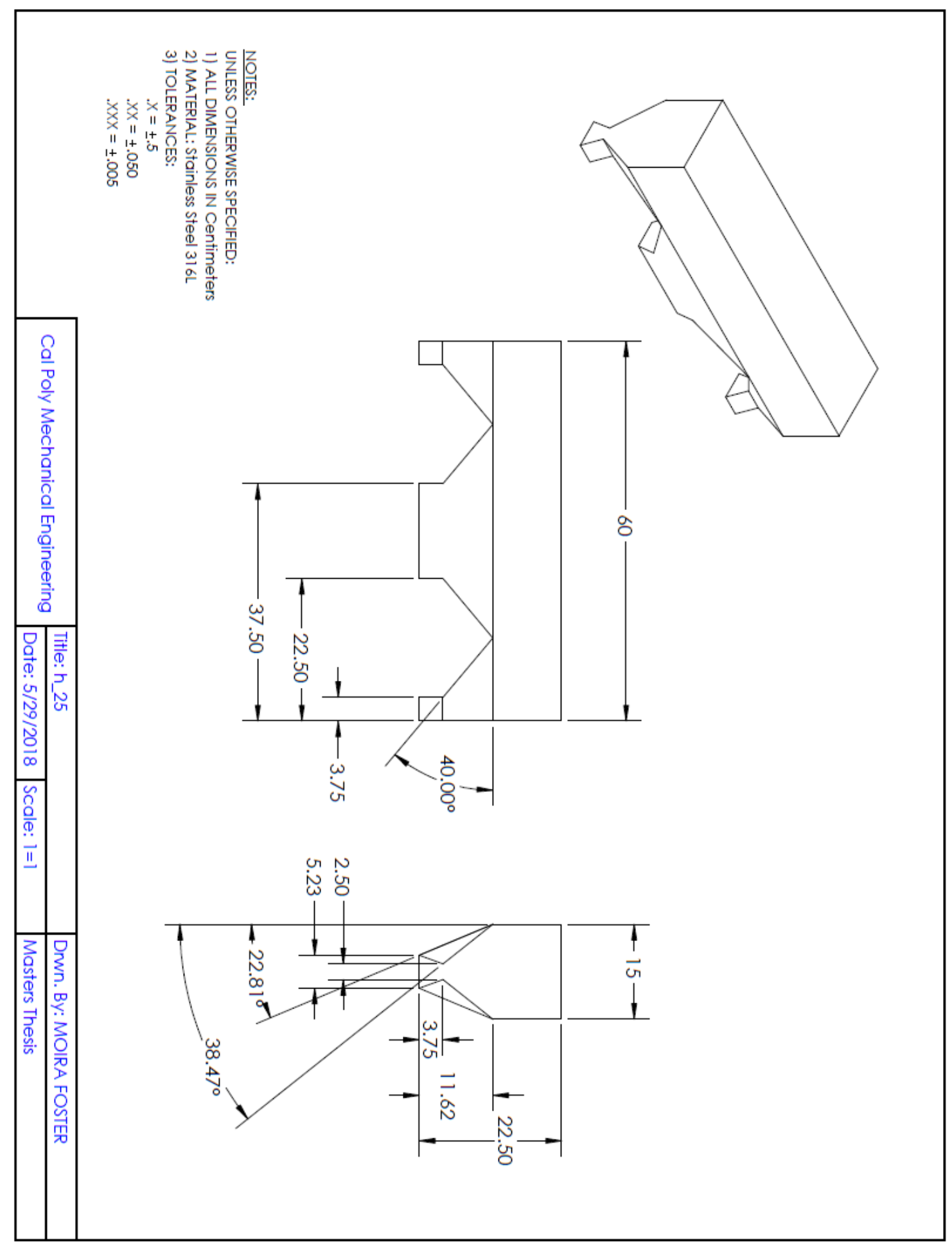




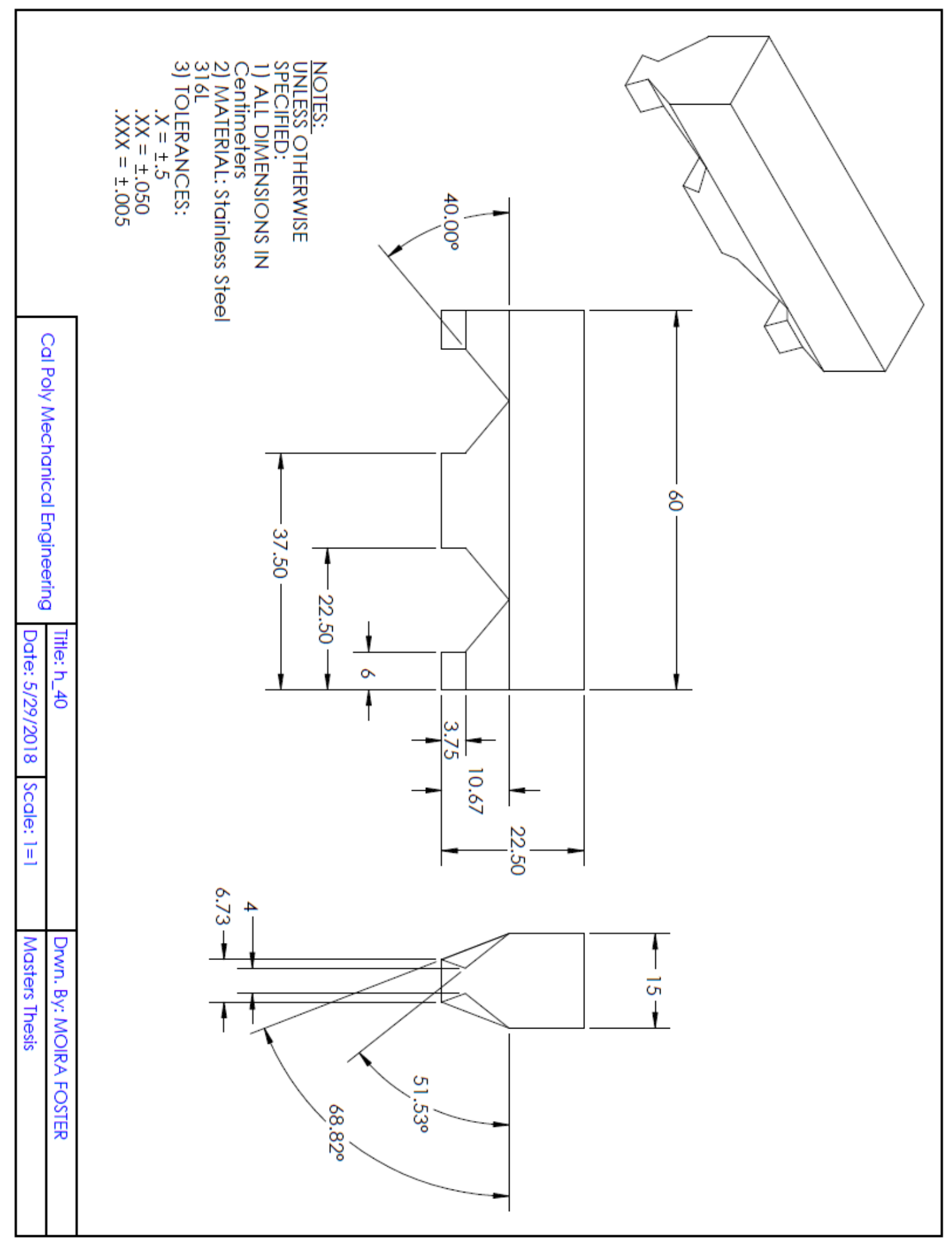




\title{
Appendix C: Matlab Codes
}

\author{
CodeA_v1.m
}

$\%$ Moira Foster

$\%$ April 2018

$\%$ This code combines and replaces importing_v5 and ZoomedIn Importing

$\%$ Codes. Use this code when you have raw data. Then proceed with Zoomed In

$\%$ Code, or other analysis.

clc

clear all

close all

PartNumber $=56$;

filter $=0.03$

designation = '13_'; \% this is mostly for 0056 with other stuff 'A_.' If don't have a designation, just put "

Filename = '3.13.18_0056_1h15_1h25_1h40_12500';

prompt = 'Does this files High mat file already exist? $(\mathrm{Yes}(1) / \mathrm{No}(0)):$;

skip = input(prompt);

$\% \%$ Run this if High matrix does not exist

if skip $==0$

$\%$ Create a database with data from the run

disp('Creating a database')

$\% \mathrm{ds}=$ datastore(['E: IOther Datal' Filename '.csv'], 'NumHeaderLines',59);

ds = datastore(['E:100' num2str(PartNumber) ' datal' Filename

'.csv'],'NumHeaderLines',59); \% Enter path and number of headerlines

Preview $=$ table $2 \operatorname{array}(\operatorname{read}(\mathrm{ds}))$

$\mathrm{L}=$ length(Preview $(1,:)) ; \%$ Number of Columns in data

$\mathrm{n}=$ numpartitions(ds); \% Number of paritions in data 
$\%$ Read in the data with a filter of 0.1 . This will show you any significant spikes.

disp('Getting data bigger than the cutoff filter')

cutoff $=$ filter; $\%$ minimum abs value of data to keep. This gets rid of smaller data points with little acceleration that I don't care about.

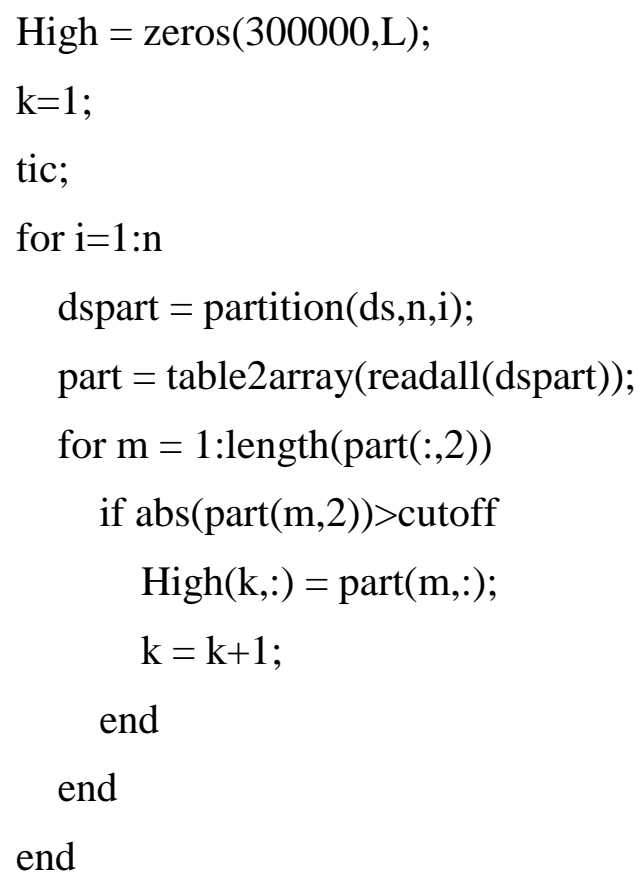

TimeHigh $=$ toc

$\%$ Save the data

disp('Saving the newly created High Array')

save(['00', num2str(PartNumber),'_High_', designation, num2str(cutoff), '.mat'],'High', 'L','ds', 'designation','PartNumber')

end

$\% \%$ Skip to here if you already have read in data and just want to load it.

if skip $==1$

cutoff $=$ filter;

load(['00', num2str(PartNumber),'_High_', designation, num2str(cutoff), '.mat'])

end

$\% \%$ Looking at all of the data in High 
figure

plot(High(:,1), High(:,2),'.')

xlabel('seconds')

ylabel('g')

title('Profile of a Print with Filter')

$\% \%$ User inputs which interval they want

prompt = 'What is the initial time you are interested in (in seconds)? Ti =';

$\mathrm{Ti}=$ input(prompt);

prompt = 'What is the final time you are interested in (in seconds)? Tf =';

Tf = input(prompt);

$\% \%$ Picking our Partition

disp('Picking the correct parition')

$\%$ how many paritions does the data have?

$\mathrm{n}=\operatorname{numpartitions}(\mathrm{ds})$;

$\%$ How long is the data overall? In seconds

endds $=\operatorname{partition}(\mathrm{ds}, \mathrm{n}, \mathrm{n})$;

endtable $=$ readall(endds $)$;

endarray $=$ table 2 array $($ endtable $)$;

Ttotal = endarray $($ end, 1$)$;

$\mathrm{L}=$ length(endarray $(1,:))$;

$\%$ What fraction of data are we investigating?

frac $=($ Tf-Ti $) /$ Ttotal;

$\mathrm{nf}=\operatorname{round}(1 / \mathrm{frac}) ; \%$ number of paritions

if $\mathrm{nf}==0$

$\mathrm{nf}=1$;

end

$\mathrm{z}=\operatorname{ceil}(\mathrm{nf} *(\mathrm{Tf}+\mathrm{Ti}) /(2 * \mathrm{Tt}$ total $)) ; \%$ Which parition we want

zoomds $=$ partition $(\mathrm{ds}, \mathrm{nf}, \mathrm{z}) ; \%$ Pulling our parition 
$\% \%$ Reading the datastore

disp('Reading the datastore')

zoomtab = readall(zoomds);

zoom = table2array $($ zoomtab $)$;

$\% \%$ Plot zoom to do a quick overview and mental check. Check if good interval value.

figure

$\operatorname{plot}($ zoom(:,1),zoom(:,2),'.')

xlabel('Seconds')

ylabel('Accel, units unknown')

title('Zoomed in Data')

disp('Press any key to save the data')

pause

$\% \%$ Saving the Zoom file

save(['00', num2str(PartNumber),'_zoom_', designation, 'part' num2str(z),

'of',num2str(nf), '.mat',],'zoom', 'PartNumber', 'L', 'designation') 
CodeE_v1.m

$\%$ CodeE_v1.m

$\%$ Moira Foster

$\%$ Doing specific stuff that D did but not cleanly.

clc

close all

$\% \%$ Load in data, rename it, plot it

load('0056_zoom_13_27460to27490.mat')

$\%$ renaming stuff

$\mathrm{A}=\mathrm{A}$;

$\mathrm{F}=12500$;

$\mathrm{Sig}=\mathrm{A}(:, 2)$;

$\operatorname{Tim}=\mathrm{A}(:, 1)-\mathrm{A}(1,1)$;

$\%$ Plotting data

figure

plot(Tim, Sig,'.')

xlabel('Seconds')

title('Raw Data')

ylabel('Acceleration (g)')

$\% \%$ Zooming in farther

prompt='What to pick new data? Yes(1)/no(0): ';

new $=$ input(prompt);

if new $==1$

prompt='What is the first time you are interested in? ti = ';

$\mathrm{ti}=$ input(prompt);

prompt='What is the last time you are interested in? $\mathrm{tf}=$ ';

$\mathrm{tf}=$ input(prompt); 
prompt='What will the title of the figures be?input with single quotes: ';

TiT = input(prompt);

else

end

$\%$ Cutting data just down to what you want.

$\mathrm{z} 0=$ find $(\operatorname{Tim}(:, 1)>=\mathrm{ti}, 1) ;$

$\mathrm{z} 1=$ find $(\operatorname{Tim}(:, 1)>=\mathrm{tf}, 1) ;$

Timmini $=\operatorname{Tim}(z 0: z 1)$;

Sigmini $=\operatorname{Sig}(z 0: z 1)$;

figure

plot(Timmini,Sigmini)

ylabel('Acceleration $(\mathrm{g})$ ')

xlabel('Seconds')

title(TiT)

$\% \%$ Wavelet

if input('Want to do Wavelet? $\mathrm{Y}(1) / \mathrm{N}(0): ')==1$

figure

cwt(Sigmini,F)

title(TiT)

ylabel('Frequency in Log [kHz]')

end

$\% \%$ Shock Response Spectrum (SRS)Prep

$\%$ function from Irvine on Matlab file exchange

if input('Want to prep for SRS?Y(1)/N(0)') == 1

$\%$ plotting the data to use

figure

plot(Timmini,Sigmini)

xlabel('seconds')

title(TiT);

ylabel('Acceleration') 
$\%$ Creating the Accel

Accel = [Timmini,Sigmini $]$;

end

$\% \%$ FFT on FFT page, Same thing different plot

if input('Want to do FFT? $\mathrm{Y}(1) / \mathrm{N}(0): ')==1$

$\mathrm{x}=$ Sigmini;

$\mathrm{L}=$ length $(\mathrm{x})$;

$\mathrm{Fs}=\mathrm{F}$;

$\mathrm{Y}=\mathrm{fft}(\mathrm{x})$;

\%Compute the two-sided spectrum P2. Then compute the single-sided spectrum P1 based on P2 and the even-valued signal length L.

$\mathrm{P} 2=\operatorname{abs}(\mathrm{Y} / \mathrm{L})$;

$\mathrm{P} 1=\mathrm{P} 2(1: \mathrm{L} / 2+1)$

$\mathrm{P} 1(2$ :end-1) $=2 * \mathrm{P} 1(2$ :end -1$)$;

$\%$ Define the frequency domain $\mathrm{f}$ and plot the single-sided amplitude spectrum P1. The amplitudes are not exactly at 0.7 and 1 , as expected, because of the added noise. On average, longer signals produce better frequency approximations.

figure

$\mathrm{f}=\mathrm{Fs} *(0:(\mathrm{L} / 2)) / \mathrm{L}$;

plot(f,P1)

title('Single-Sided Amplitude Spectrum of X(t)')

xlabel('f (Hz)')

ylabel('|P1(f)|')

$\%$

figure

$\mathrm{f}=\mathrm{Fs} *(0:(\mathrm{L} / 2)) / \mathrm{L}$;

semilogx(f,P1,'.')

title('Single-Sided Amplitude Spectrum of X(t). SemiLog Plot') 
xlabel('f (Hz)')

ylabel('|P1(f)|')

$x \lim ([10,100000])$

end 